Linköping Studies in Science and Technology Thesis No. 1730

\title{
Image Analysis and Visualization of the \\ Human Mastoid Air Cell System
}

\author{
Olivier Cros
}

Department of Biomedical Engineering

Linköping University, Sweden

Linköping, October 2015 


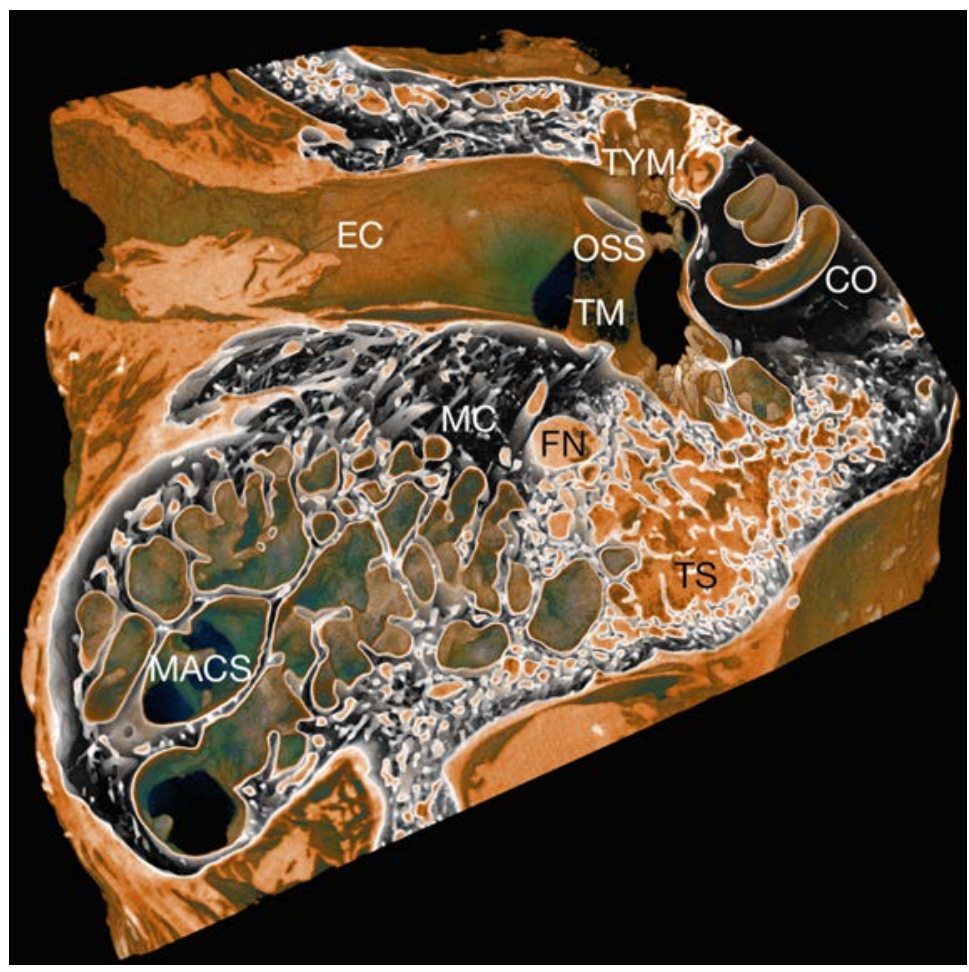

Legend: CO cochlea, EC ear canal, FN facial nerve, MACS mastoid air cell system, MC micro-channels, OSS ossicles (manubrium of the incus), TM tympanic membrane, TS trabecular spaces, TYM tympanum (middle ear).

\section{Image Analysis and Visualization of the \\ Human Mastoid Air Cell System}

(C) 2015 Olivier Cros

Department of Biomedical Engineering Linköping University, Sweden 


\section{Abstract}

From an engineering background, it is often believed that the human anatomy has already been fully described. Radiology has greatly contributed to understand the inside of the human body without surgical intervention. Despite great advances in clinical CT scanning, image quality is still related to a limited amount X-ray exposure for the patient safety. This limitation prevents fine anatomical structures to be visible and, more importantly, to be detected. Where such modality is of great advantage for screening patients, extracting parameters like surface area and volume implies the bone structure to be large enough in relation to the scan resolution.

The mastoid, located in the temporal bone, houses an air cell system whose cells have a variation in size that can go far below current conventional clinical CT scanner resolution. Therefore, the mastoid air cell system is only partially represented on a CT scan. Any statistical analysis will be biased towards air cells of smaller size. To allow a complete representation of the mastoid air cell system, a micro-CT scanner is more adequate. MicroCT scanning uses approximately the same amount of X-rays but for a much longer exposure time compared to what is normally allowed for patients. Human temporal bone specimens are therefore necessary when using such scanning method. Where the conventional clinical CT scanner lacks level of minutes details, micro-CT scanning provides an overwhelming amount of fine details.

Prior to any image analysis of medical data, visualization of the data is often needed to learn how to extract the structures of interest for further processing. Visualization of micro-CT scans is of no exception. Due to the high resolution nature of the data, visualization of such data not only requires modern and powerful computers, but also necessitates a tremendous amount of time to adjust the hiding of irrelevant structures, to find the correct orientation, while emphasising the structure of interest. Once the quality of the data has been assessed, and a strategy for the image processing has been decided, the image processing can start, to in turn extract metrics such as the surface area or volume and draw statistics from it. The temporal bone being one of the most complex in the human body, visualization of micro-CT scanning of this bone awakens the curiosity of the experimenter, especially with the correct visualization settings.

This thesis first presents a statistical analysis determining the surface area to volume ratio of the mastoid air cell system of human temporal bone, from micro-CT scanning using methods previously applied for conventional 
clinical CT scannings. The study compared current results with previous studies, with successive downsampling the data down to a resolution found in conventional clinical CT scanning. The results from the statistical analysis showed that all the small mastoid air cells, that cannot be detected in conventional clinical CT scans, do heavily contribute to the estimation of the surface area, and in consequence to the estimation of the surface area to volume ratio by a factor of about 2.6. Such a result further strengthens the idea of the mastoid to play an active role in pressure regulation and gas exchange.

Discovery of micro-channels through specific use of a non-traditional transfer function was then reported, where a qualitative and a quantitative preanalysis was performed are described. To gain more knowledge about these micro-channels, a local structure tensor analysis was applied where structures are described in terms of planar, tubular, or isotropic structures. The results from this structural tensor analysis, also reported in this thesis, suggest these micro-channels to potentially be part of a more complex framework, which hypothetically would provide a separate blood supply for the mucosa lining the mastoid air cell system. 


\section{Acknowledgements}

Many people have contributed to this thesis, directly or indirectly. First, I am grateful for my employer, the department of Otolaryngology, Head and Neck Surgery, at Aalborg Hospital South in Aalborg, Denmark. Without the financial support and great patience, I would not be here today. I will obviously try to return this big favour to the department.

I would like to particularly thank MD \& PhD Michael Gaihede, my main clinical supervisor, for your constant support and providing this amazing knowledge you have about the ear both from an anatomical but also from a physiological point of view. I must admit, it is not everyday that I met a clinician who is eager to spend his free time to break down all secrets of the mastoid during late evening conversations. It is always a pleasure. I am actually proud that you recently called me "mister Mastoid". Simona, you are not forgotten either, I hope we can get to work more in the near future about this famous mastoid bone.

I would also like to thank Professor Hans Knutsson, my main technical supervisor, for being a never ending source of new ideas and inspiration. Thank you also Dr. Mats Andersson for endless discussions about filter design, snorkelling, motorcycles, coffee, beer brewing, and motivating me during tough periods from personal matters but also when in doubt with my academic career. I will miss you but I also know you will be in good hand in your new position. Thanks to Dr. Anders Eklund, my new co-supervisor, for your already endless support and our daily talks.

Thank you Professor Magnus Borga for your early supervision. The same applies to the Center for Medical Image Science and Visualization (CMIV) and I am happy to share my knowledge during seminars. Also, thanks to my colleagues and the personnel at the department of biomedical engineering for always being kind and helpful.

I would also like to acknowledge the people at the centre for X-ray Tomography, Department of Physics and Astronomy, University of Ghent, Belgium; especially Elin Pawels and Manuel Dierick and their colleagues. Without you, this $\mathrm{PhD}$ would definitely have turned in a different direction.

I also want to greet my friends, especially Anders Eklund, Filipe Marreiros, Patrick Bennysson, Rafael Sanchez without whom I would have felt quite alone on the daily basis. You have been a great support directly and indi- 
rectly.

All my gratitudes to my family, especially my parents Jacques and Nicole Cros, for always being supportive and engaged in my research. Without you I would not be where I am. Dad, I hope you are in peace where you are now, and I miss you terribly. You have no idea how much I learned from you and I stil carry your principles in me. Mum, I never met someone so brave in relation to fighting all problems you went through in your life. You are so modern, so fun, while being an amazing good mother. Thanks to my both sisters Véronique Poupon and Nathalie Pesson and their respective families. It is always nice to see you despite the distance that separates us.

Olivier Cros. Linköping, XXXX 2015. 


\section{Table of Contents}

1 Introduction 1

1.1 Introduction . . . . . . . . . . . . . . . . . . 1

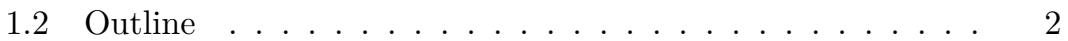

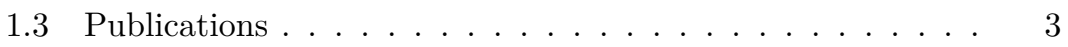

1.4 Abbrevations .................. . . 4

2 General Anatomy \& Physiology 5

2.1 Introduction . . . . . . . . . . . . . . . . 5

2.2 Anatomy of the Temporal Bone . . . . . . . . . . . 6

2.2 .1 The outer ear . . . . . . . . . . . 8

2.2 .2 The middle ear . . . . . . . . . . . . . . . . . . . 9

2.2 .3 The inner ear . . . . . . . . . . . . . . 10

2.2.4 The Eustachian tube . . . . . . . . . . . . . . 12

3 The Mastoid bone $\quad \mathbf{1 5}$

3.1 General description . . . . . . . . . . . . . . 15

3.1.1 Personal interpretation of the origin of the mastoid air cells in the newborns . . . . . . . . . . . . 17

3.1.2 Level of pneumatization . . . . . . . . . . . . 20

3.1.3 Pressure regulation and gas exchange . . . . . . 20

4 Clinial vs Micro-CT Scanning 23

4.1 Introduction . . . . . . . . . . . . . . . . . . 23

4.1.1 Clinical X-ray CT scanner . . . . . . . . . . 25

4.1.2 Micro-CT scanner . . . . . . . . . . . . . 29

4.1.3 Estimated price . . . . . . . . . . 31

5 Image Processing 33

5.1 Introduction . . . . . . . . . . . . . . . . . . 33

5.2 Basic image processing . . . . . . . . . . . . . . . 33

5.2.1 Pixels and voxels . . . . . . . . . . . . . . 33

5.2 .2 Grayscale content of an image. . . . . . . . . 36

5.2 .3 Image noise . . . . . . . . . . . . . . . . 37

5.2 .4 Convolution . . . . . . . . . . . . . . . . . . . 38

5.2 .5 Noise filtering . . . . . . . . . . . . . . 40

5.2.6 Segmentation by thresholding . . . . . . . . . . . . 41

5.2.7 Morphology on binary images . . . . . . . . . . 44 
5.2.8 Masking original data over a binary segmentation . . 47

5.2.9 Measuring surface area and volume . . . . . . . . 48

5.3 More advanced image processing . . . . . . . . . . . . . 50

5.3 .1 Filter design . . . . . . . . . . . . . . . . 53

5.3 .2 The quadrature filter . . . . . . . . . . . . . 59

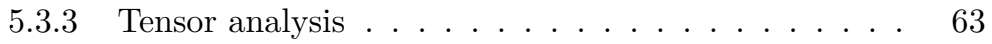

5.4 Volume rendering . . . . . . . . . . . . . . . . . 65

6 Contributions of this thesis $\quad 69$

6.1 Introduction . . . . . . . . . . . . . . . 69

6.2 Contribution 1. Surface area and volume of the mastoid air cell system . . . . . . . . . . . . . . . 69

6.3 Contribution 2. Discovery of micro-channels within the mastoid bone . . . . . . . . . . . . . 77

6.4 Contribution 3. Structual analysis of the micro-channels . . 82

7 Summary of Papers $\quad 91$

7.1 Introduction . . . . . . . . . . . . . . . . . . . . . 91

7.2 Paper I - Mastoid structural properties determined by analysis of high-resolution CT scanning . . . . . . . . . . . . . 91

7.3 Paper II - Micro-channels in the mastoid anatomy. Indications of a separate blood supply of the air cell system mucosa by micro-CT scanning . . . . . . . . . . . . . . . . . . 92

7.4 Paper III - Structural analysis of micro-channels in human temporal bone . . . . . . . . . 92

$\begin{array}{lll}8 & \text { Discussion } & 93\end{array}$ 


\section{Introduction}

"The process of scientific discovery is, in effect, a continual flight from wonder."

- Albert Einstein

\subsection{Introduction}

Similarly to the lungs, the rate of gas exchange carried out by the mastoid process in the temporal bone is influenced by the mucosal surface area [37]. This is particularly the case for the mastoid bone with its complex air cell system with cells varying sizes and shapes. An impaired gas exchange, besides a malfunctioning Eustachian tube, results in a negative middle ear pressure; i.e. both in the tympanum and in the mastoid air cell system. Negative middle ear pressure leads to different middle ear diseases such as the otitis media with effusion, cholesteatoma invading all the airspaces in the middle ear, or retractions pockets in the tympanum [36]. As stated in [12], understanding the mechanism of the middle ear pressure regulation is important for both physiologists and practising clinicians; notably the practising clinicians in their decisions on how to treat their patients.

The surface area of the mucosa in the middle ear, especially the one covering the mastoid air cell system, is therefore a valuable parameter for physiological studies of gas exchanged between the air cells and the capillaries present in the mucosa lining the air cells [11][12][36].

Quantitative measurement of the entire mastoid air cell system aeration is, however, still reported in few studies [9][37][42]. A plausible explanation of this sparse literature emanates from the fact that there is no technique to allow a direct measurement of the mucosa surface area for the entire mastoid air cell system in vivo. An alternative is to scan the mastoid bone through X-ray computed tomography and consider the walls of the mastoid air cell system as a surrogate to the very thin mucosa invisible on clinical 
CT scans. The volume of gas occupied within the mastoid air cells, also important to estimate when investigating how well a mastoid is pneumatized, is though easier to estimate using X-ray computed tomography and reported in several studies [4][9][20][27][29][37][41][46].

Recent advances in micro-CT scanning technology allows scanning such complex structures using very high resolution, in turn producing more accurate statistics. The aim of this work is to investigate the use of microCT scanning of human temporal bone specimens to estimate the surface area to volume ratio using classical image processing methods. Compare with results from previous studies where conventional clinical CT scans were used, and finally assessing whether the obtained estimates help further understand the anatomy and physiology of the mastoid air cell system. Micro-channels were discovered while visualizing the temporal bone specimens using different visualization settings. A structural analysis of micro-channels within the bone was therefore assessed.

\subsection{Outline}

The thesis is divided into 7 parts. The thesis is formed in a way such that non-medical readers can obtain a brief and basic introduction to the anatomy and physiology of the human temporal bone, including all its components beside the mastoid bone (chapter 2). Chapter 3, alone, describes the mastoid bone and its air cell system, as it is the main focus of this study. Chapter 4 gives a brief comparison between a conventional clinical CT scanner and a micro-CT scanner built in Ghent (Belgium), that was used to produce the processed scans. This chapter can be of use for both clinical and technical readers not familiar with parameters important when aiming at good quality scans. For non-technical readers, chapter 5 introduces some necessary background about image processing, needed to understand some of the notions used in the papers. While readers with a clinical background can skip chapters 2 and 3, readers involved in the field of image processing can skip chapter 5. Chapter 6 states the contributions of this thesis from both clinical and technical aspects. Chapter 7 contains a summary of the papers, while chapter 9 provides a discussion about the overall work and ideas for future work.

N.B.: It should be noted that besides Figs. 2.1, 2.2, 2.4, 2.5, 2.6, 2.7, 2.8, 2.9, 3.1, 3.2, 3.3, 4.1, 4.2, 4.3, 4.5, 5.38, 6.12, 6.13, all remaining illustrations presented in this thesis were produced by the sole author Olivier Cros. Permission to use these illustrated should be asked beforehand. 


\subsection{Publications}

I Olivier Cros, Hans Knutsson, Mats Andersson, Elin Pawels, Magnus Borga, Michael Gaihede

Mastoid structural properties determined by analysis of high-resolution CT scanning

Under revision.

II Olivier Cros, Magnus Borga, Elin Pawels, Joris JJ Dirckx, Michael Gaihede

Micro-channels in the mastoid anatomy. Indications of a separate blood supply of the air cell system mucosa by micro-CT scanning Published in Hearing Research Special Issue MEMRO 2012, Volume 301, pages 60-65, 2013.

III Olivier Cros, Michael Gaihede, Mats Andersson, Hans Knutsson Structural analysis of micro-channels in human temporal bone Accepted for poster presentation at the annual meeting of the International Symposium on Biomedical Imaging (ISBI), New York, United States of America, April, pages 9-12, 2015. 


\subsection{Abbrevations}

This table lists some of the abbreviations that are used in this thesis, along with their meanings.

$\begin{array}{ll}\text { CT } & \text { Computed Tomography } \\ \text { MAC } & \text { Mastoid Air Cell } \\ \text { MACS } & \text { Mastoid Air Cell System } \\ \text { ME } & \text { Middle ear } \\ \text { TYMP } & \text { Tympanum } \\ \text { TM } & \text { Tympanic membrane } \\ \text { OM } & \text { Otitis media } \\ \text { EC } & \text { Ear canal } \\ \text { ET } & \text { Eustachian tube } \\ \text { SNR } & \text { Signal to Noise Ratio }\end{array}$




\section{General Anatomy \& Physiology}

"Non sibi sed omnibus."

Not for myself but for all.

\section{$2.1 \quad$ Introduction}

Before introducing the temporal bone as a whole entity, taxonomy of anatomical terms and spatial positions is briefly introduced with the first six positions illustrated isolated from the others for correspondence purpose with Fig. 2.1.

- Lateral towards the outside,

- Medial towards the midline,

- Anterior towards the front,

- Posterior towards the back,

- Superior towards the top,

- Inferior towards the bottom,

- Endo- inner part of a structure,

- Meso- middle part of a structure,

- Ecto- outer part of a structure,

- Exo- external to a structure,

- Epi- outside a structure,

- Hypo- under the structure,

- Hyper- over the structure,

- Inter- between structures,

- Intra- within a structure,

- Peri- surrounding a structure,

- Retro- behind a structure.

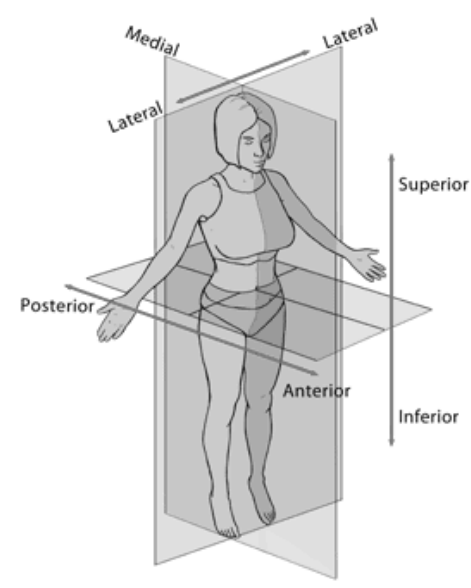

Figure 2.1: Anatomical directions.

These terms are used alternatively, globally or locally. As a simple example, the temporal bone is located laterally in relation the human skull, while the 
cochlea is located medially in relation to the temporal bone. Combination of locations are also possible as, for instance, the superior retrosigmoid air cells depicting air cells located at the superior part and at the back of the mastoid process. A structure located at the periphery of another structure is for example called the lateral perisinusal cells, meaning air cells at the periphery of a sinus located in the back of the mastoid towards the lateral side of the bone. As a side note, mastoid air cells located at the centre of the mastoid can also be named medial or central air cells, while air cells located towards the outside of the mastoid bone will be called lateral or peripheral air cells. This notion should be kept in mind while reading the thesis. Also to further help the reader, taxonomy for the orientation of medical images are resumed in the following list and represented graphically in Fig. 2.2.

- Axial: superior $\leftrightarrow$ anterior.

- Coronal: anterior $\leftrightarrow$ posterior.

- Sagittal: left $\leftrightarrow$ right.

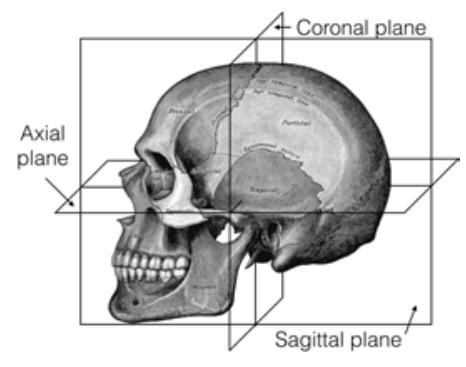

Figure 2.2: Anatomical planes.

\subsection{Anatomy of the Temporal Bone}

The temporal bone houses the organ of hearing. The temporal bone consists of five parts: the temporal squamosa, the petrous portion, the mastoid process, the tympanic bone, and the styloid process, see Fig. 2.3.

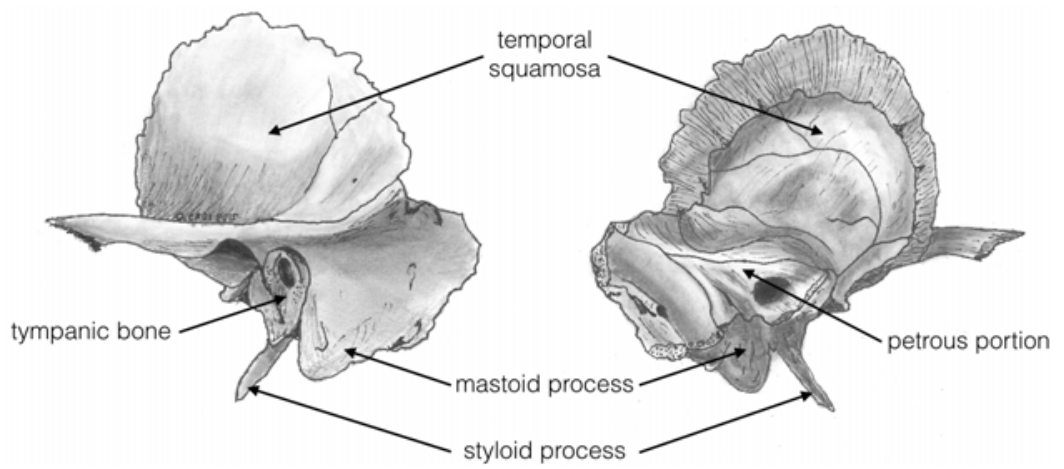

Figure 2.3: Temporal bone viewed alone from a lateral side (left) and a medial side (right), respectively viewed from the outside and the inside. 
The temporal squamosa (from Latin squama, "scale"-shape structure) is the biggest part of the temporal bone, lying superior to all parts. The petrous portion of the temporal bone, located inside the skull, is recognizable with its pyramidal shapes housing the middle ear and the inner ear. The word petrous originates from the Latin word petrosus, and relates to the very hard portion of the bone housing the internal auditory organs. The mastoid process, from the new Latin "mastoides" resembling a nipple or breast, is located posteriorly and inferiorly to the squamous part.

The tympanic bone is a small portion situated inferior to the temporal squamosa, anterior to the mastoid process, and superior to the styloid process. The styloid process runs inferior to the tympanic bone, and is shaped like a thorn pointing downwards. It is used as an anchor point for several muscles. The described structures are illustrated in Fig. 2.3.

A coronal section of a right temporal bone reveals the complexity inside the temporal bone, see Fig. 2.4.

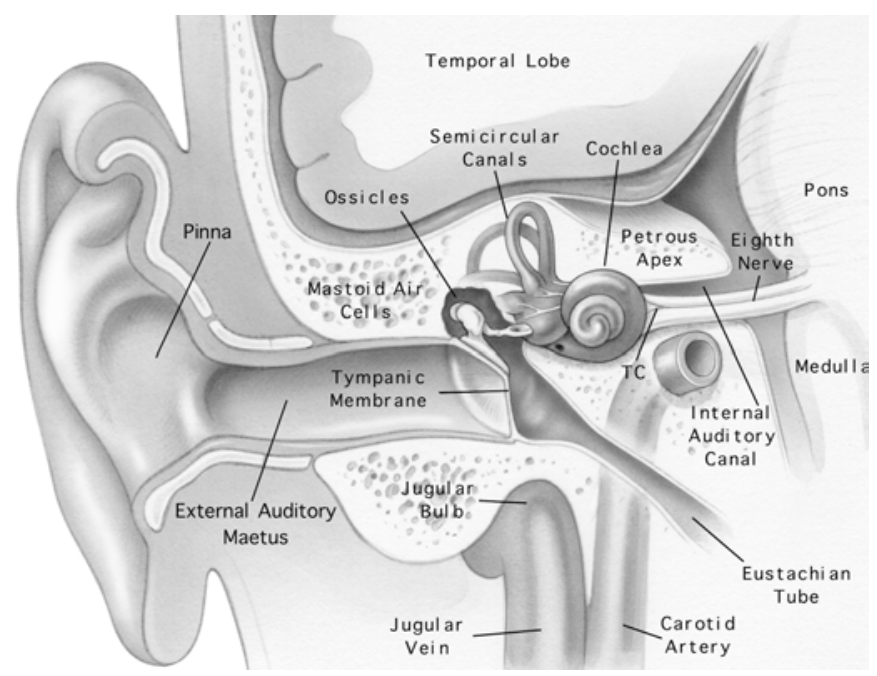

Figure 2.4: Cut through a temporal bone to reveal all its internal structures. Source: Atlas of Skull Base Surgery 85 Neurotology, Thieme, 2009. Image copyrighted by RK Jackler. Permission granted for non-profit educational use.

From a physiological point of view, the temporal bone can be decomposed into three main parts: the external ear, the middle ear, and the inner ear. These parts are described in the following sections. 


\subsubsection{The outer ear}

The outer ear is the external portion of the ear, which consists of the pinna, the external auditory meatus (also known as the ear canal), and the tympanic membrane, see Fig. 2.5.

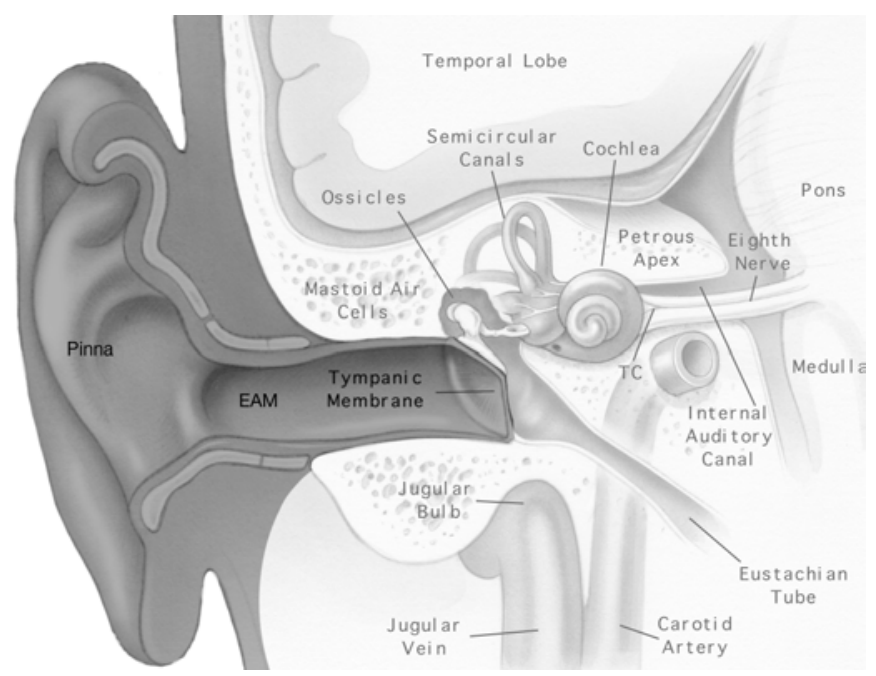

Figure 2.5: The outer ear. Legend EAM: external auditory maetus. Source: Atlas of Skull Base Surgery \& Neurotology, Thieme, 2009. Image copyrighted by RK Jackler. Permission granted for non-profit educational use.

The pinna, also known as the auricle, is the visible portion that is generally referred to as "the ear". Its function is to localize sound sources and direct sound into the ear. The folds of the pinna allow some specific frequencies to be amplified, while other frequencies can be damped.

The external auditory meatus (EAM), also named the external auditory canal or simply the ear canal, extends from the pinna to the tympanic membrane and has a length of about 26 millimetres $(\mathrm{mm})$ with a diameter of about $7 \mathrm{~mm}$. The size and shape of the ear canal vary among individuals and for both ears.

The tympanic membrane, also known as the eardrum, is a cone-shaped structure separating the outer ear from the middle ear and protects the middle and inner ear from foreign objects. The tympanic membrane resonates in response to sound pressure waves. The displacement during vibration is extremely small, about one-billionth of a centimetre. 


\subsubsection{The middle ear}

The middle ear cavity is a combination of two cavities, the tympanum and the mastoid air cell system, see Fig. 2.6. The mastoid air cell system will be explained in more details in the next chapter, and therefore only the tympanum is briefly detailed here. The tympanum is the narrow air-filled space of the middle ear, where the ossicles are located. The tympanum resembles an oblique rectangular room with a floor, a ceiling, and four walls.

Sound waves traveling through the ear canal will hit the tympanic membrane. This wave information travels across the air-filled tympanum via a series of delicate bones called the ossicles, see Fig. 2.7. The ossicles are composed of the malleus (also known as hammer), incus (also known as anvil) and stapes (also known as stirrup), see Fig. 2.7. They form an ossicular chain.

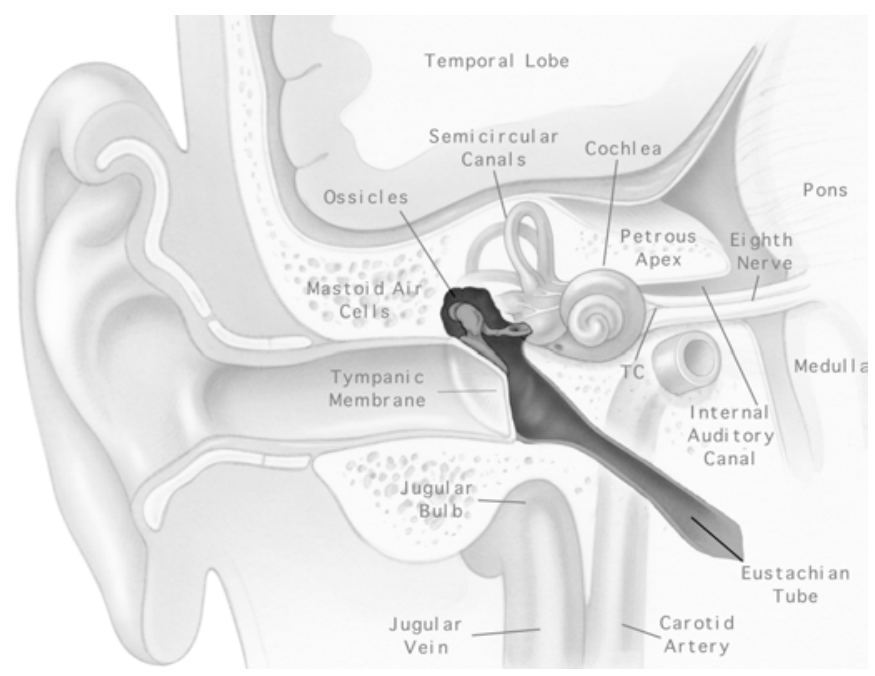

Figure 2.6: The middle ear. Source: Atlas of Skull Base Surgery \& Neurotogy, Thieme, 2009. Image copyrighted by RK Jackler. Permission granted for non-profit educational use.

While the handle of the malleus, also known as the manubrium, articulates with the tympanic membrane, the footplate of the stapes articulates with the oval window, a membrane-covered opening which leads to the vestibule of the inner ear, located at the opposite side of the tympanum. Tendons attaching the head of the malleus and the incus are attached to the tegmen tympani, a bony wall separating the cranial cavity from the superior part of the tymapnum, the epitympanic recess or attic, see the vertical tendons in Fig. 2.7. Behind the head of the malleus and towards its upper part, 


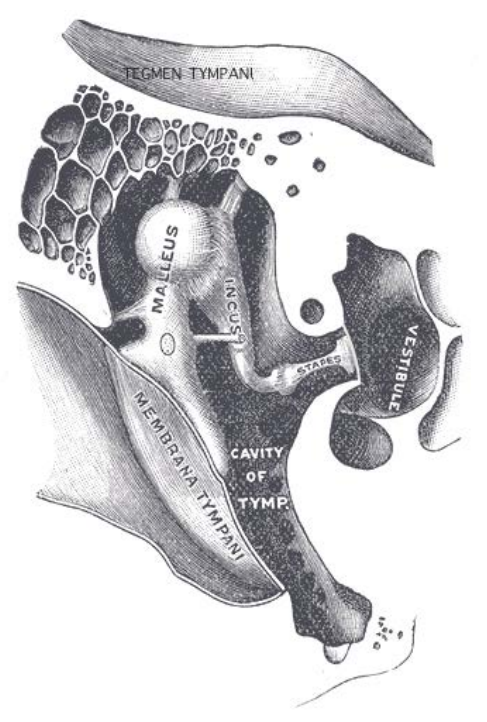

Figure 2.7: The ossicular chain with the malleus in direct contact with the tympanic membrane, and the stapes viewed laterally attached to the oval window separating the vestibule from the tympanum. The incus transmits the movements induced by the malleus to the stapes. Also, notice the tendons attaching the ossicles to the tympanum. Source: http://commons.wikimedia.org/wiki/File:Gray919.png.

the posterior wall of the tympanum is mostly occupied by the aditus ad antrum, a path towards the mastoid air cell system via the antrum.

\subsubsection{The inner ear}

The inner ear, shown in Fig. 2.8, can be divided into two labyrinths: a bony labyrinth and a membranous labyrinth.

The osseous labyrinth consists of the cochlea, the vestibule, and the semicircular canals, a series of bony cavities within the petrous temporal bone. These bony cavities are lined with periosteum and contain perilymph; extracellular fluid at the periphery of the structure. The oval window, articulated by the footplate of the stapes, is an opening in the lateral wall of the vestibule of the osseous labyrinth.

The membranous labyrinth is composed of communicating membranous sacs and ducts housed within the osseous labyrinth. It is cushioned by the surrounding perilymph and contains the endolymph within its confines; an extracellular fluid inside the structure. The membranous labyrinth also houses a cochlear, a vestibular, and semicircular canals components. 


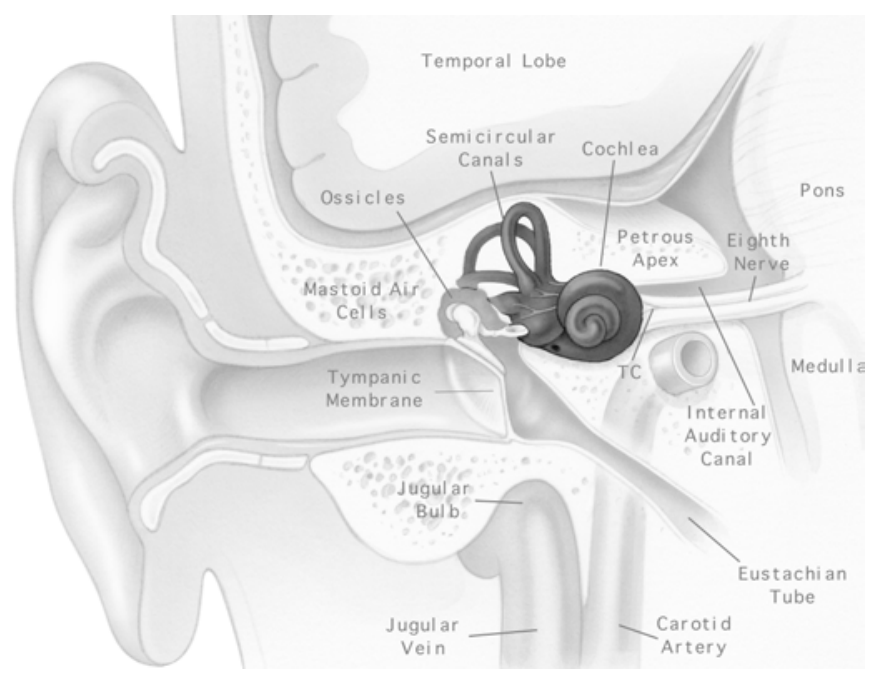

Figure 2.8: The inner ear. Source: Atlas of Skull Base Surgery 85 Neurotogy, Thieme, 2009. Image copyrighted by RK Jackler. Permission granted for non-profit educational use.

The cochlea

The cochlea is the auditory portion of the inner ear. The spiral-shaped cavity within the cochlea has three fluid-filled sections along its main axis: the scala vestibuli, the scala tympani, and the cochlear duct. The organ of Corti, located in the cochlear duct on the basilar membrane, transforms mechanical waves into electric signals sent to the neurons in the brain.

The vestibule

The vestibule is the central part of the osseous labyrinth. The vestibular apparatus is composed of the utricle and the saccule. They respectively sense linear acceleration in the horizontal and vertical planes. Within these organs are hair cells. The cilia of these cells are intricately associated with a membranous substance containing calcium carbonate granules, or "otoliths", also commonly known as stones in the cochlea. Movement of the head induces a shearing of the hair cells by the mobile otoliths. This directional change is sensed by the brain via the superior division of the vestibular nerve at the utricle, and via the inferior division of the vestibular nerve to the saccule. Together, the otolithic organ organs of both ears are of prime importance for directional sensation. 


\section{The semi-circular canals}

The third main apparatus in the inner ear is the set of three semi-circular canals. Each stands at right angles in relation to each others. The superior, posterior, and lateral semicircular canals are located posterior and superior to the vestibule.

\subsubsection{The Eustachian tube}

The Eustachian tube originates in the posterior part of the nose, runs slightly uphill, and enters the tympanum inferiorly, see Fig. 2.9 (left).
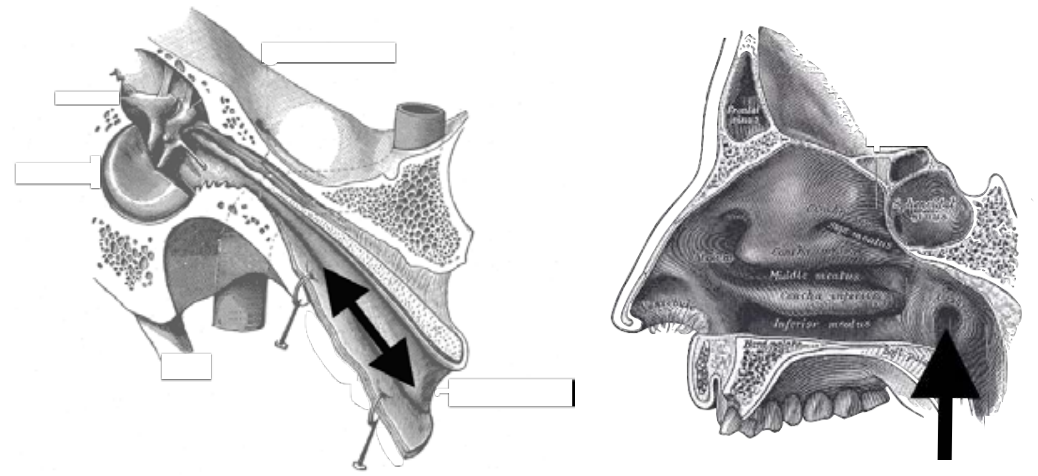

Figure 2.9: Course of the Eustachian tube from the middle ear (left) and its entrance from the nasal cavity (right), see the red vertical arrow. Source: left. Hill, M.A. (2015) Embryology Gray0915.jpg, right: [2].

The cartilage provides a supporting structure for two thirds of the Eustachian tube, while the part closest to the tympanum is made of bone. The tissue lining the Eustachian tube is similar to that inside the nasal cavity, and may respond in the same way (swelling) when presented with similar stimuli.

\section{Function of the Eustachian tube}

The primary function of the Eustachian tube is to ventilate the middle ear space, ensuring that its pressure remains at near normal environmental air pressure; known as middle ear pressure. The secondary function of the Eustachian tube is to drain any accumulated secretions, infections, or debris from the tympanum.

Several small muscles located in the back of the throat and palate control the opening and closing of the tube. Swallowing and yawning cause contractions of the muscles located in the back of the throat, and facilitate 
the regulation of the Eustachian tube function. If it was not for the Eustachian tube, the middle ear cavity would be an isolated air pocket inside the head which would be vulnerable to every change in air pressure, underand over-pressure, leading to a pathological middle ear.

Normally, the Eustachian tube is closed, which helps to prevent the inadvertent contamination of the middle ear space by the normal secretions found in the back of the nose. A dysfunctional Eustachian tube can lead to chronic ear infections. A much more common problem is a failure of the Eustachian tube to effectively regulate air pressure.

Partial or complete blockage of the Eustachian tube can cause sensations of popping, clicking, and ear fullness and occasionally moderate to severe ear pain. Such intense pain is most frequently experienced when sudden air pressure changes arise while traveling by airplane, particularly during take-off or landing.

As Eustachian tube function worsens, air pressure in the middle ear falls, and the ear feels full and sounds are perceived as muffled. Eventually, a vacuum is created which can then cause fluid to be drawn into the middle ear space (termed serous otitis media). If the fluid becomes infected, a common ear infection (suppurative otitis media) will eventually be developed [41].

\section{Mucosa lining the tympanum and the Eustachian tube}

Mucosa is the innermost layer of hollow organs. All bone surfaces of the ME cleft are covered with a mucosa. The structure of the mucosa is subdivided in an epithelial layer (mucosal epithelium) located on the top of a basal membrane (also called basal lamina), and a connective tissue layer called lamina propria, adherent to the the outer part of the underlying bone; the periosteum layer [43].

The lamina propria is formed of loose connective tissue, characterized by a less organized appearance and by being composed of relatively few cells (mezenchymal cells, basal cells, fibroblasts, lymphocytes, macrophages, etc) in a large volume of extracellular matrix.

The major matrix components are collagen and elastic fibres. Generally, the loose connective tissue contains many blood vessels, nerve endings, lymphatic vessels and, interestingly, misses a basal membrane making it easier to be crossed by macromolecules and cells [43].

Starting from the nasopharynx (located between the mouth and the nose; see Fig. 2.9 (right image to the left of the black arrow)), the epithelium is respiratory, with one to three layers of columnar or cubical cells, often ciliated or with microvili, secretory and non-secretory, and intercalated with 
goblet cells [39]. The role of these ciliated cuboidal cells is to carry waste towards the Eustachian tube.

The antero-inferior of the tympanum (on the frontal part and on the lower part), is structurally similar to the mucosa from nasopharynx and the eustachian tube. In the postero-superior part (the back part of the tympanum on the top part) of the tympanic cavity and the mastoid, the epithelium is mono-layered, flat, alternating with very rare cuboidal cells [17]. The postero-superior and antrum mucosa seems also to be more abundant with superficial blood vessels, compared to the tympanic cavity [5].

The common structures have now been introduced, and the next chapter is solely dedicated to the mastoid bone and the mastoid air cell system, along with some information about the mucosa lining the mastoid air cell system. 


\section{The Mastoid bone}

"Natura ingenium disecta cadavera pandit."

- The cadaver dissection demonstrates the wisdom of nature.

\subsection{General description}

The mastoid bone is a conical prominence on the lateral side of the temporal bone, located at the posterior level of the ear canal, see Fig. 3.1.

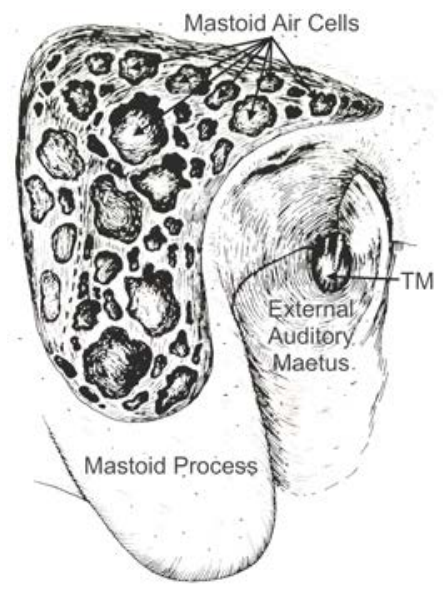

Figure 3.1: Exposed mastoid on the lateral side reflecting the mastoid air cells present within the bone. Abbreviation: (TM) Tympanic membrane [44]

From an external point of view, the mastoid bone serves as an attachment point for several muscles - the splenius capitis, longissimus capitis, digastric posterior belly, and sternocleidomastoid. Inside the mastoid bone, a very large number of interconnected air cells of different size and shapes are 
carved into the bone. Altogether, these air cells form the so-called mastoid air cell system. The mastoid air cell system directly communicates with the tympanum via the epitympanum by the aditum ad mastoid. Together, the mastoid air cell system and the tympanum form the middle ear. An axial view of temporal bone allows a better representation of the communication between the tympanum and the mastoid air cell system, see Fig. 3.2.

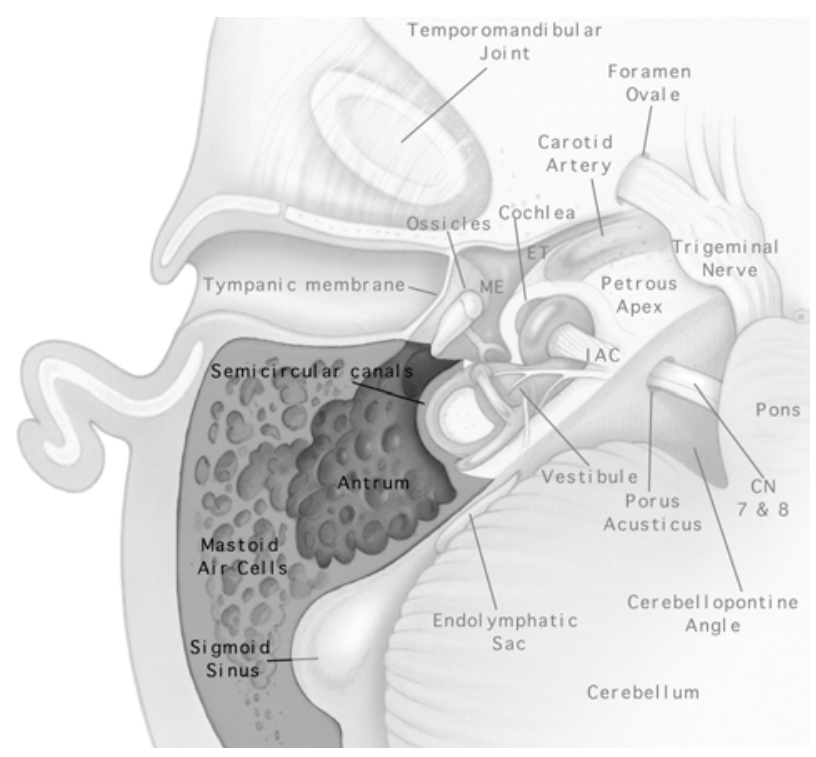

Figure 3.2: Axial section of the temporal bone revealing the mastoid bone. Source: Atlas of Skull Base Surgery 83 Neurotology, Thieme, 2009. Image copyrighted by RK Jackler. Permission granted for non-profit educational use.

The mastoid air cell system is categorized according to various sub-regions. These sub-regions are listed below, and are illustrated in Fig. 3.3.

- Antral - anterior to the antrum at the proximity and towards the ear canal,

- Periantral - at the periphery of the antrum,

- Tegmental - located at the level of the tegmen tympani at the superior level of the mastoid bone above the ear canal,

- Lateral and medial zygomatic - at the level of the zigomatic bone laterally and medially in relation to the external auditory canal,

- Sinodural - at the postero-superior level of the mastoid in the vicinity of the cranial cavity,

- Central mastoid - all air cells immediately inferior to the cells in the mastoid antrum, 
- Superior retrosigmoid - at the supero-posterior part of the mastoid bone near the sigmoid sinus,

- Medial and lateral Perisinusal - all air cells at the periphery of the sigmoid sinus towards the medial and lateral parts of the mastoid process,

- Inferior retrosigmoid - at the infero-posterior part of the mastoid bone near the sigmoid sinus,

- Perifacial - at the mid level of the posterior wall of the external ear canal,

- Medial and lateral apical - at the tip level of the mastoid process respectively towards the anterior and posterior walls of the external ear canal and towards the pinna.

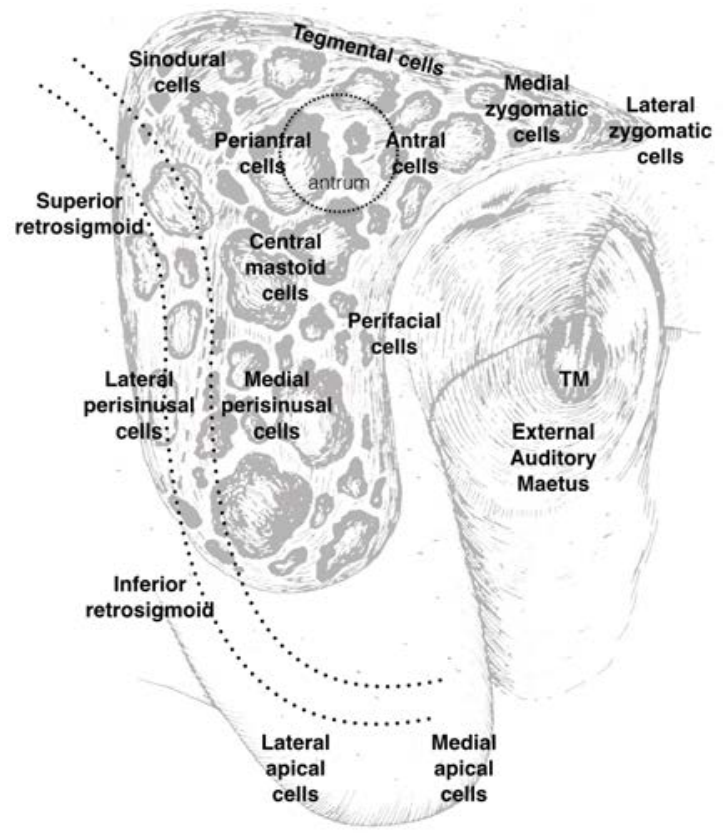

Figure 3.3: The mastoid air cells categorized into different substructures. Inspired from M. Tos illustration.

\subsubsection{Personal interpretation of the origin of the mastoid air cells in the newborns}

In the newborn, the mastoid bone is practically one single air space, later on named the antrum, surrounded by diploic bone, i.e. mostly containing marrow. As the mastoid process develops, the marrow space hollows out and the air cell system develops, while becoming lined with a highly vascular cuboidal epithelium layer emanating from the endoderm. This cuboidal 
epithelium will later on form the mucosa of the mastoid air cell system. The endoderm is one of the three primary layers in the very early embryo.

The air cells grow out primarily from the attic of the tympanum to the aditus ad antrum, creating a complex structure of cavities separated by bony walls communicating with each other, see Fig. 3.4 (A and B) for two different stages of pneumatization.

Similarities with the alveoli of the lungs, where extensive gas exchange takes place, have been found due to a close contact between the mucosa and the blood vessels, see Fig. 3.4 (C). This has lead to the mastoid air cell system being known as a mini-lung.

A.

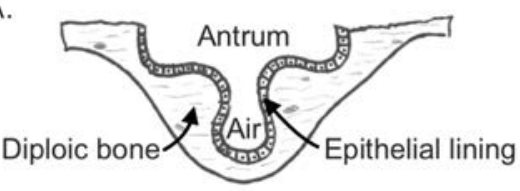

B.
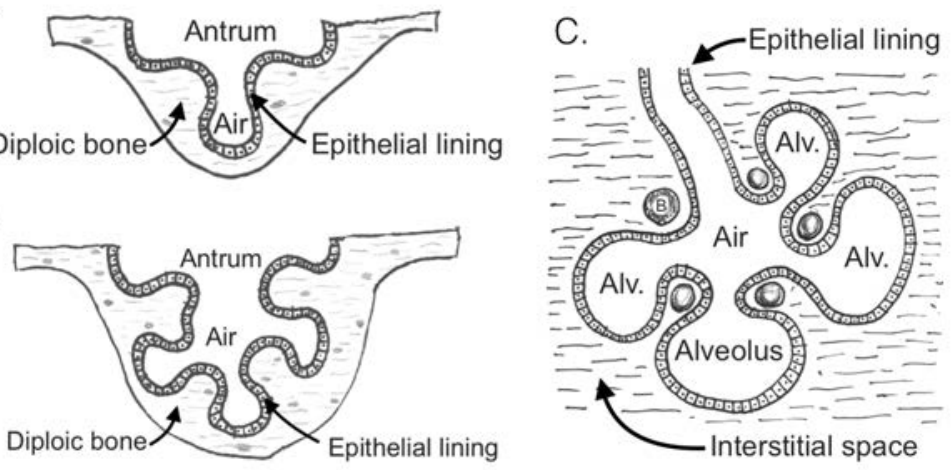

Figure 3.4: $A$. E B B. represent two different stages where the epithelial lining covers the newly formed air cells during the pneumatization process. C. gives a simplified illustration of a set of alveoli formed by an epithelial lining and surrounded by interstitial space with blood vessels at the proximity for gas exchange. Abbreviation in C.: (B) blood vessel, (Alv.) alveolus.

A broader representation of the growth of the mastoid air cell system is illustrated in Fig. 3.5. As can be observed, the mastoid air cell system originates from the tympanum, and spreads over time. 


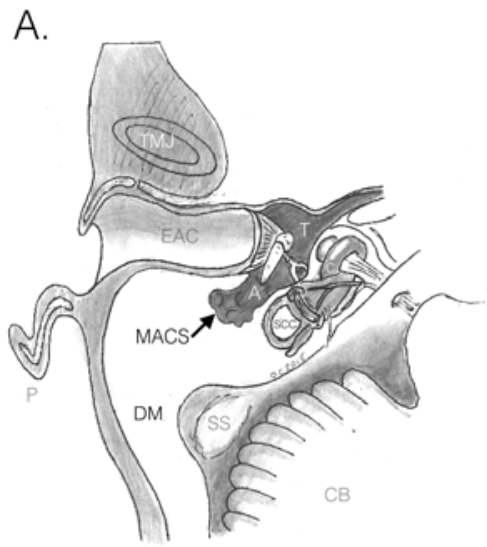

C.

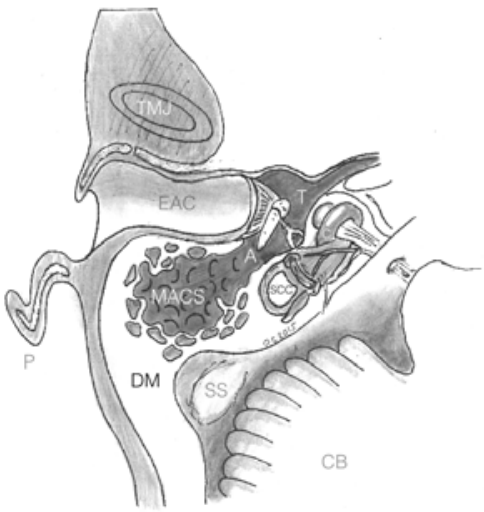

B.

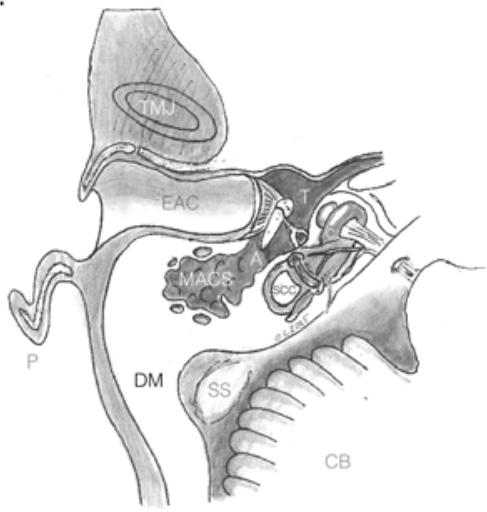

D.

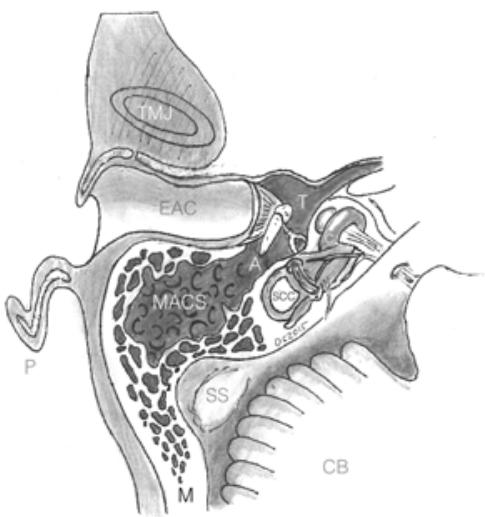

Figure 3.5: Transverse plane of the hearing system. A. First stage: single air cell later called antrum, B. Beginning of the cellularization, C. Enlargement of the mastoid air cell system, D. Completion of the pneumatization with small air cells at the periphery of the main MACS. Abbreviations: (A) antrum, (CB) cerebellum, (DM) diploic mastoid, (EAC) external auditory canal, $(M)$ mastoid, $(P)$ pinna, $(S C C)$ semi-circular canals, (SS) sigmoid sinus, (T) tympanum, (TMJ) temporomandibular joint.

According to [30], the mastoid air cell system serves both as a reservoir of air and as a buffer system to replace the air in the middle ear cavity when the pressure becomes negative. However, the buffer capacity depends heavily on the level of pneumatization of the mastoid bone, as explained below.

A very interesting study has been carried out to simulate a morphogenetic model of the cranial pneumatization, based on an invasive tissue hypothesis. Zollikofer et al. simulated the invasion of airspaces in cranial bone along 
with the mucosa lining the airspaces [50]. Not only did they simulate the growth of the mastoid air cell system by a mathematical model, but also indirectly showed how the airspaces can be constrained by surrounding structures. This would explain the possible variation of the shape of the mastoid air cells, in relation to their location within the bone.

\subsubsection{Level of pneumatization}

The mastoid air cell pneumatization can be divided into 3 different types of pneumatization:

- sclerotic where the pneumatization is absent,

- diploic where the pneumatization is poor due to the presence of bone marrow inside the air cells,

- pneumatic where the pneumatization is complete.

Combined with a dysfunctional Eustachian tube, a poor pneumatization of the mastoid air cell system may lead to middle ear infections, and eventually to the development of otitis media, englobing different inflammatory diseases in the middle ear.

\subsubsection{Pressure regulation and gas exchange}

In organisms, gas exchange is carried on by diffusion governed by the Fick's law. The principal factors behind Fick's law are the surface area of the membrane where diffusion occurs, the thickness of the membrane, the concentration gradient, and the speed at which molecules diffuse.

According to [41], the tympanum is essentially a single large air-cell. However, whereas the mucosa covering the tympanum respects many of the conditions for an efficient gas exchange, the surface area to volume ratio is limited by the tympanum principally being a single large air cell, see Fig. 3.6 .

Opposite to the tympanum, the mastoid air cell system presents a very complex structure with a very intricate surface, especially at the level of the antrum from where most air cells emanate, see Fig. 3.7.

The walls of each individual air cell often appear smooth. However, when observing the locations where the air cells split from each other, these conducts display spicules and columnar structures which greatly increase the surface area in relation to the overall volume of the mastoid air cell system. 

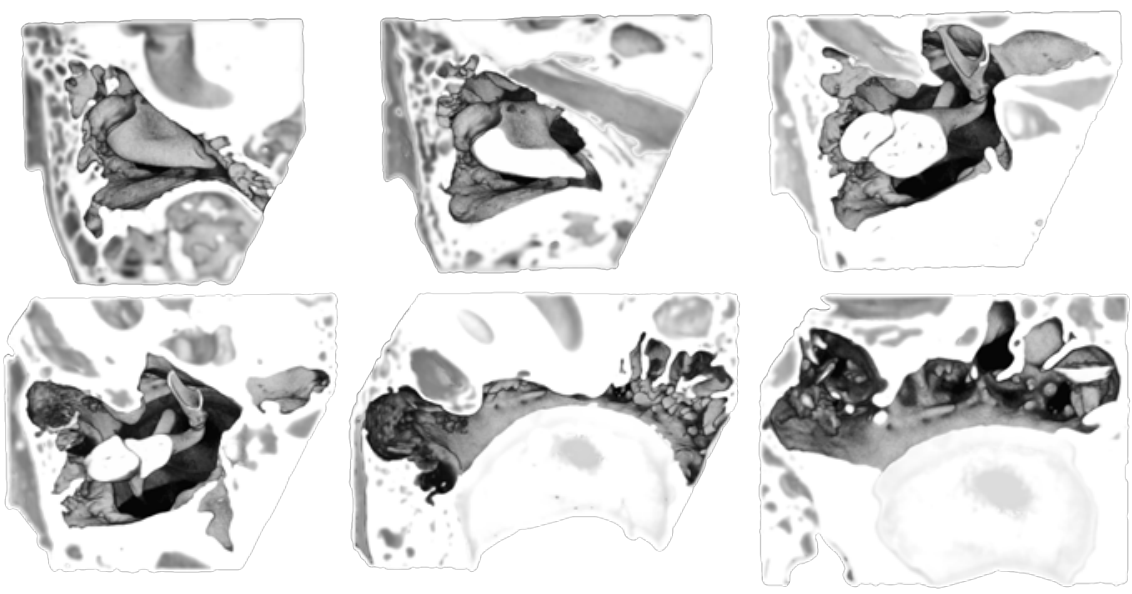

Figure 3.6: Enhanced representations of the tympanum at different depths, to expose the regularity of its walls viewed from top to bottom. The data was maximally cropped around the tympanum, using clipping planes, to mostly concentrate on the tympanum itself, allowing a better viualization.

\section{Mucosa lining the mastoid in relation the tympanum}

Compared to the mucosa lining the tympanic cavity, only a few studies concerning the histological properties of the mastoid mucosa have been reported. In only two scientific publications the structure of the human mastoid mucosa has been compared to the mucosa lining the tympanum and Eustachian tube. Only histological samples where taken from the antrum part of the mastoid [6] [34].

While it is believed that the mucosa of the tympanic cavity in its anteroinferior part is more specialized in clearance, the mucosa of the mastoid would facilitate an efficient gas exchange by a significantly shorter diffusion distance, and higher perfusion compared with the tympanic cavity [5] [18].

The next chapter gives a short comparison between a clinical CT scanner commonly used in public hospital with a micro-CT scanner built at the department of Physics and Astronomy in Ghent (Belgium). 

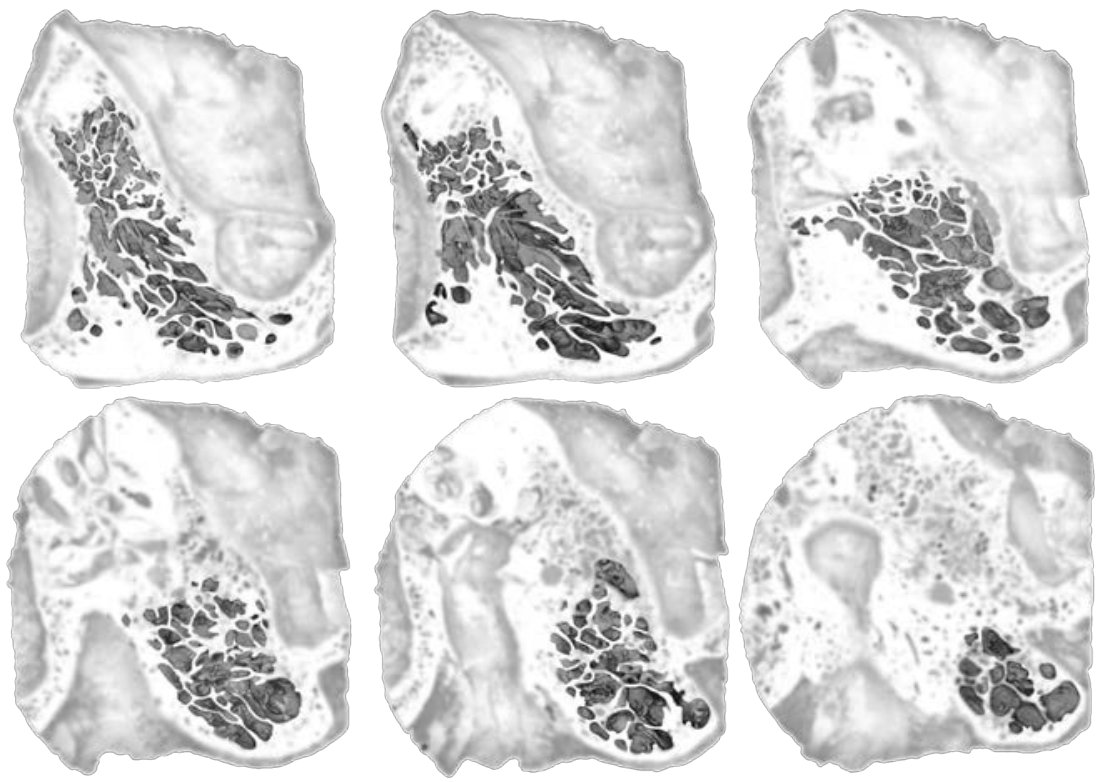

Figure 3.7: Representations of the mastoid air cell system at different depths, to expose its shape complexity viewed from top to bottom. 


\title{
Clinial vs Micro-CT Scanning
}

\author{
"Pedibus usque ad caput." \\ From head to toe.
}

\subsection{Introduction}

Both clinical CT and micro-CT scanners use X-ray technology. Before stating the differences between these two types of scanning, common features are discussed.

X-ray Computed Tomography (CT) imaging consists of exposing an object with X-rays at multiple orientations, while measuring the intensity decay of X-rays when going through different materials. This decrease in intensity is described in terms of the X-ray energy, the length of the X-rays path, and the coefficients directly related to the material linear attenuation. From the signals generated during X-ray attenuation, reconstruction using specialized algorithms is necessary to produce the final image data.

The principal components of an X-ray tomography system are:

- an X-ray source,

- a series of detectors that measure X-ray intensity attenuation usually located on the opposite side of the X-ray source in relation to the scanned object,

- a rotating device either housing the X-ray source or on which the scanned sample is spinning.

Most X-ray CT machines, especially in the clinical world, use an X-ray tube. Other X-ray sources, such as a synchrotron or a gamma-ray emitter can also be used.

Relevant characteristics concerning the X-ray tube are listed below: 
- the target material,

- the peak X-ray energy,

- the current expressed in Ampere,

- the focal spot size directly impacting the spatial resolution of the final scan data.

There exists three main configuration types. As illustrated in Fig. 4.1 (A), the X-ray are collimated in a linear fashion and collected by a linear detector array resulting in a so-called planar beam type of scanning. In Fig. 4.1 (B), parallel-beam scanning is performed using a synchrotron beam line as an X-ray source. In cone-beam scanning, Fig. 4.1 (C), the linear array is replaced by a planar detector, and the beam is no longer collimated. Compared to Fig. 4.1 (A), cone-beam X-ray, parallel-beam scanning does not have a collimator restricting the X-ray path.

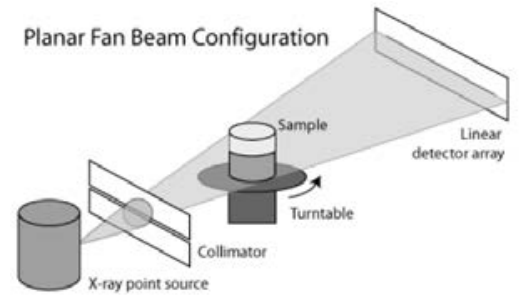

A

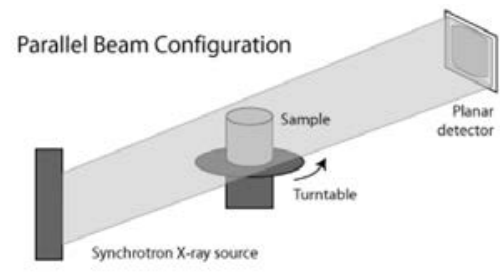

B

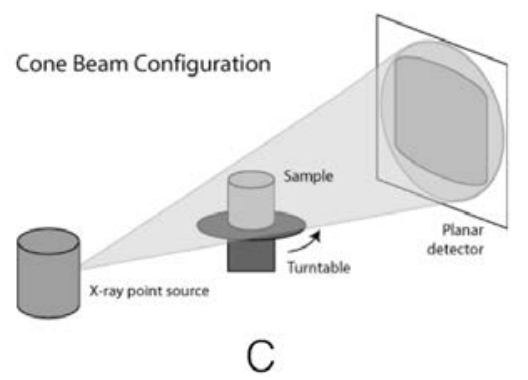

Figure 4.1: Three types of scanner: (A) Planar Fan Beam, (B) Parallel Beam, (C) Cone Beam [1].

In the case of a planar fan-beam configuration, scattering of the X-rays, producing spurious additional X-rays outside the path going from X-ray source and the detector, can be reduced using collimators. Such linear arrays are more efficient than planar ones, such as in Fig. 4.1 (B and C), at the expense of producing a single slice per scan. The aperture of the linear array sets the thickness of the resulting slice. To get a $3 \mathrm{D}$ volume, the scanned object needs to be moved normal to the path formed between the X-ray source and the linear detector. 
For the parallel-beam type of scanning, a synchrotron beam line is used as the X-ray source. A good feature of parallel-beam scanning is the lack of distortion in the resulting data. However, the width of the X-ray beam limits the size of the object to be scanned. Synchrotron radiation generally has very high intensity leading to a quick acquisition of the data and objects with a size of up to $6 \mathrm{~cm}$ in diameter can be imaged.

In the case of a cone-beam CT scanner, a planar detector replaces the need for a collimator. The data is acquired during a single rotation and reconstructed into images using a cone-beam algorithm. Some of the downsides of a cone-beam CT scanner, are the blurring and distortion of the data at the extremities of the object being scanned. When using high energy X-ray for better resolution, X-ray scattering artefacts also hampers the final data.

There exist variants from these three CT configurations, such as multipleslice acquisition in which a planar detector is used but where the generated data are reconstructed using a fan-beam reconstruction algorithm, along with spiral scanning where the sample is displaced during the acquisition.

\subsubsection{Clinical X-ray CT scanner}

A conventional clinical CT scanner typically uses a planar fan-beam type of configuration. A typical clinical X-ray CT scanner is illustrated in Fig. 4.2 .

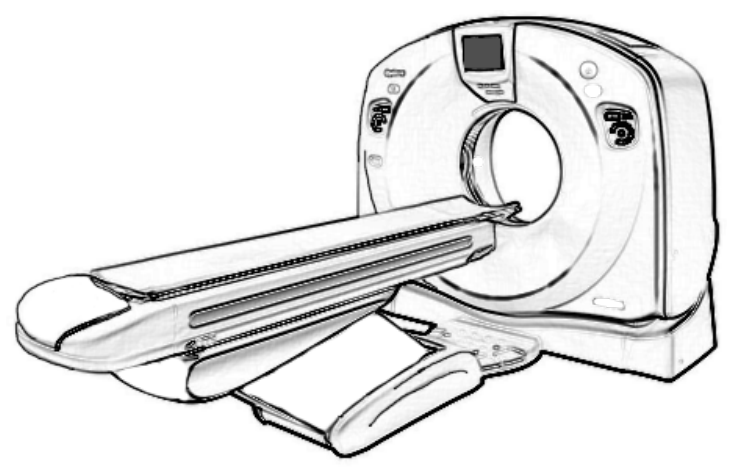

Figure 4.2: Conventional clinical CT scanner. Source: www3.gehealthcare.in with personal artistic modifications.

Clinical computed tomography is used daily for medical applications since the seventies. The bed on which the patient is lying on is sliding through a doughnut-shaped gantry. The gantry of the scanner houses the X-ray source, located on one side of the ring, and the set of detectors on the opposite side, see Fig. 4.3. The frame holding both the X-ray source and 
the detectors rotates around the patient. The succession of radiographs taken along the displacement of the bed forms a 3D image.

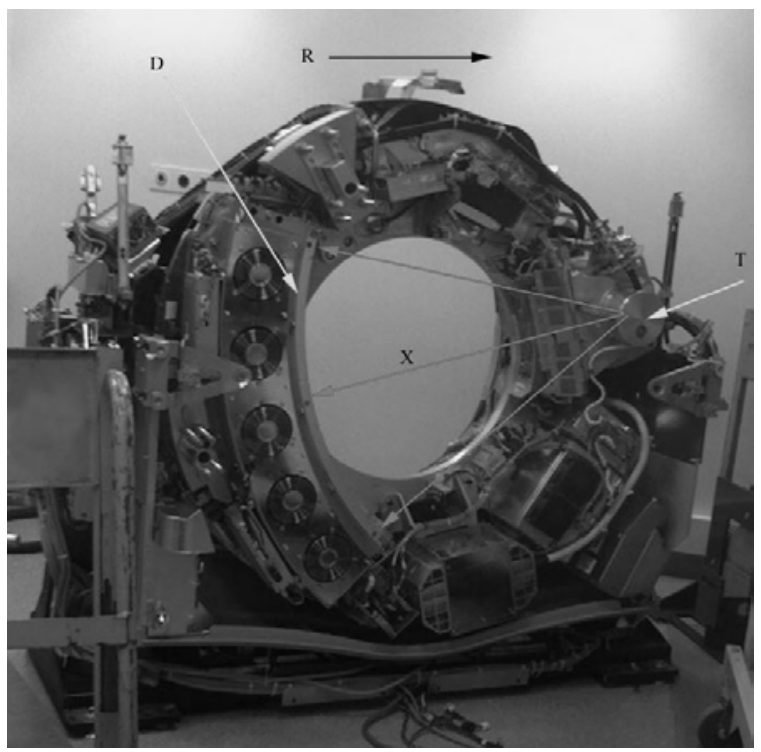

Figure 4.3: Photo of the internal part of a conventional clinical CT scanner [47]. Legend: T: X-ray tube, D: X-ray detectors, X: X-ray beam, R: Gantry rotation.

The main parameters the radiology technologist needs to fine-tune which contribute to minimizing the radiation dose are:

- the tube current (mA),

- the peak kilovoltage $(\mathrm{kVp})$,

- the pitch (degrees),

- the gantry cycle time (sec.)

The number of electrons accelerated across the x-ray source tube per unit of time defines the tube current expressed in milliampere $(\mathrm{mA})$. This parameter is not only an important factor for the resulting scan image quality but more importantly for the amount of radiation dose imposed to the patient [22]. Lowering the tube current leads to less radiation dosage, but affects the resulting scan images with an increase in the noise level [38].

The peak kilovoltage $(\mathrm{kVp})$ corresponds to the energy of the emitted $\mathrm{X}$ rays onto the patient being scanned. Each tissue type has its own range of density which will affect the X-ray beam's attenuation. Larger and/or denser objects will require a higher energy peak voltage to make sure that 
a sufficient number of photons, from the X-rays, exit the body part being scanned and then become collected by the detectors. To reduce the radiation dose imposed to the patient, the peak kilovoltage can also be reduced at the expense of an increased image noise reducing the contrast-to-noise ratio, often leading to an increase of the tube current to compensate for less noise [38].

Pitch, expressed in degrees $\left(^{\circ}\right)$, is factor mostly related to the moving table, on which the patient lays on, in a helical CT scanner. Pitch is calculated as a proportion between the table feed, expressed in centimetres per full rotation of the gantry, and the total width of collimated x-ray beam along the $\mathrm{z}$ direction [35]. When increasing the pitch while keeping the tube current per unit of time constant as the table moves, the radiation dose is decreased.

Decreasing the radiation dose can also be achieved by decreasing gantry rotation time, expressed in seconds (sec.); the faster the gantry rotation, the lower the dose. Doubling the speed of rotation per full rotation of the gantry reduces the dose essentially by half. As for the previous parameters decreasing the dose level while tuning the gantry rotation time leads to an increase in image noise.

The Scanning time is significantly longer compared to routine scans by setting both a small collimator size and a low pitch value to increase the scan resolution. The effective radiation dose for a head CT for temporal bone imaging is usually around 2 millisievert $(\mathrm{mSv})$.

Typical settings for a conventional clinical CT scan for temporal bone imaging, used when scanning the temporal bone specimens in this study, are listed below:

- Peak kilovoltage: $120 \mathrm{kVp}$

- Tube current: $131 \mathrm{~mA}$

- Time (per rotation): $1.0 \mathrm{sec}$

- Spiral pitch factor: $0.53^{\circ}$

- Overall average acquisition Time: $16 \mathrm{sec}$

- Reconstruction diameter: $135 \mathrm{~mm}$

- Distance source to detector: $949 \mathrm{~mm}$

- Distance source to patient: $541 \mathrm{~mm}$

- Table height: $56 \mathrm{~mm}$

- Exposure time: 1825 ms (about 30.5s) 
- Single collimator width: $0.625 \mathrm{~mm}$

- Reconstructed slice thickness $0.625 \mathrm{~mm}$

- Table speed: $10.625 \mathrm{~mm} / \mathrm{sec}$

- Table speed per rotation: $10.625 \mathrm{~mm} / \mathrm{sec} / 360^{\circ}$

- Start: below mastoids (bottom)

- End: clear petrous bones (top)

- Pixel spacing: X: $0.298 \mathrm{~mm}, \mathrm{Y}: 0.298 \mathrm{~mm}$

- Rows: 512 pixels

- Columns: 512 pixels

- Encoding: 16 bits unsigned.

- Mode: Helical

- Filtering: "medium filter"

- Convolution kernel: "BONEPLUS"

The scanner used during this study is a LightSpeed Pro 32 scanner from General Electrics MEDICAL SYSTEMS at the radiology department of Aalborg Hospital South. More specifications about this scanner can be found in [3]. A clinical CT scan of a patient's head as well as a clinical CT scan of a temporal bone specimen alone are illustrated in Fig. 4.4.
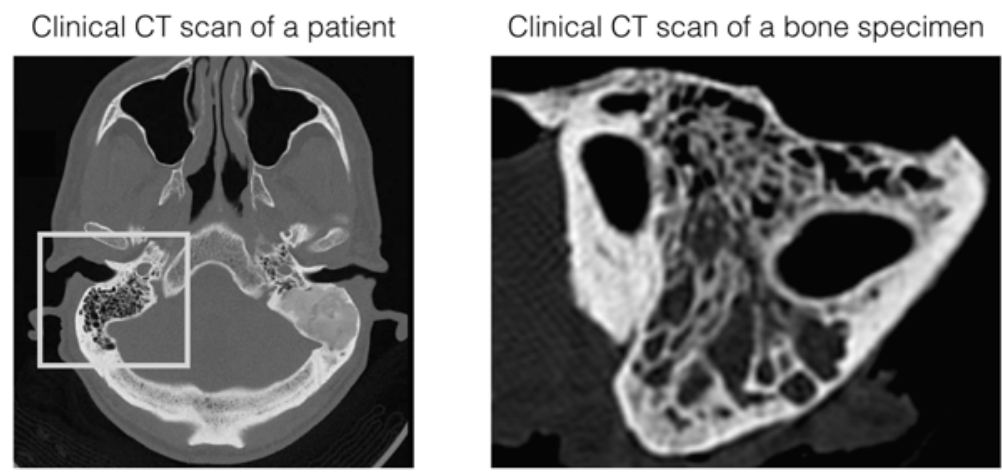

Figure 4.4: A clinical CT scan of a patient's head and of a temporal bone specimen. The yellow square reveals the mastoid of the patient of the right ear (always seen on opposite direction in CT images). Clinical CT scan of a temporal bone specimen giving more details about the mastoid air cell system through adjustment of the scanner settings.

In the next section is presented the micro-CT used during the study. 


\subsubsection{Micro-CT scanner}

Micro-CT provides much higher-resolution and quality scans than a conventional clinical CT scanner. From a general setup, instead of the X-ray source and the detectors to rotate around the patients or object to be scanned, the object itself is rotated on a turntable.

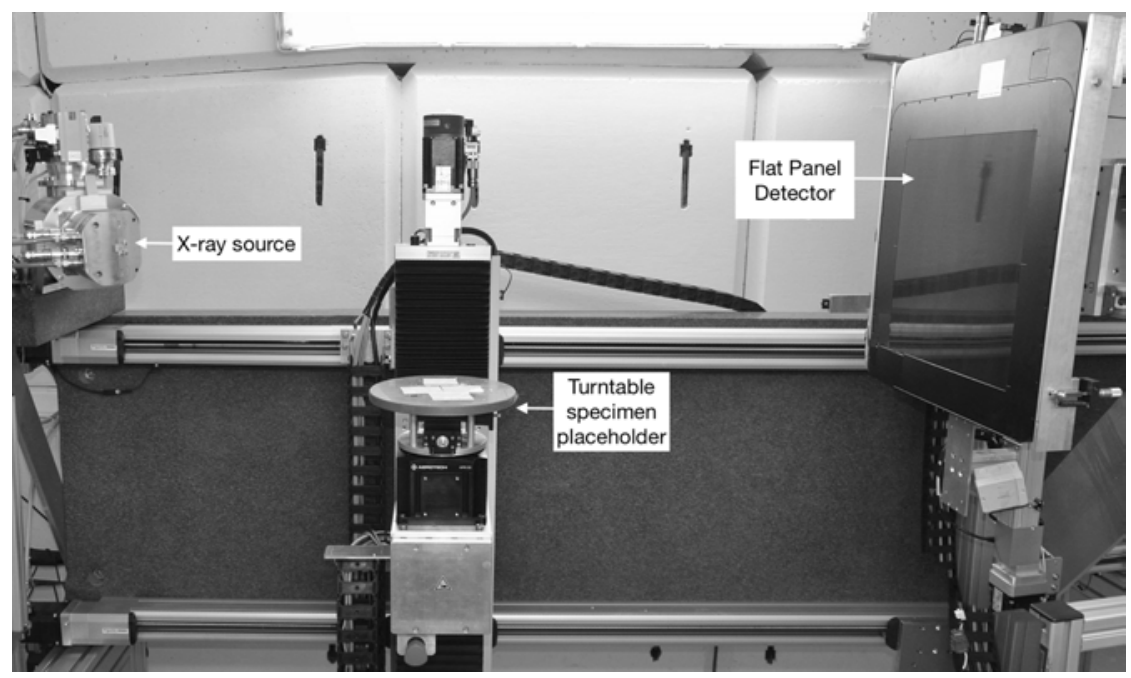

Figure 4.5: Photograph of the micro-CT scanner used in this study, from the department of Physics and Astronomy at Ghent University in Belgium [33].

In this study, a custom-built micro-CT scanner available at the department of Physics and Astronomy, Ghent (Belgium), was used for scanning of 8 bone specimens applying the scanning protocol as in [32]. Fig. 4.5 shows the micro-CT scanner with the X-ray source located to the left, the turntable placed in the middle on which the sample is carefully positioned, and the flat panel detector. Each bone specimen was positioned in a plastic cup on a computer controlled turntable, so as to avoid displacements during rotation while being scanned.

Depending on the sample size, the peak kilovoltage of the X-ray source was set to $120 \mathrm{kVp}$ with $3 \mathrm{~mm}$ of aluminium of beam filtration, see Paper 2 for the details. A full cone beam rotation scan was performed in $2 \mathrm{~s}$ of exposure per projection to ensure a high signal to noise ratio. A series of about 2100 cross-sections was reconstructed using a software package called Octopus, developed locally at the department of Physics and Astronomy in Ghent [45].

A list of the scanning parameters is given below for one of the bone specimen investigated during this study: 
- Beam power: 50 to 60 Watts

- Peak kilovoltage: 120 kVp

- Tube current: $500 \mu \mathrm{A}$

- Centre of rotation: $833.15 \mathrm{~mm}$

- Source object distance: $387.9 \mathrm{~mm}$

- Source detector distance: $1392.56 \mathrm{~mm}$

- Vertical centre: $974 \mathrm{~mm}$

- Tilt: $-0.18^{\circ}$

- Skew: $0.17^{\circ}$

- Voxel size: $0.055296 \mathrm{~mm}$ isotropic

- Rows: 1708

- Columns: 1708

- Scan volume $(\mathrm{X}, \mathrm{Y}, \mathrm{Z}): 94.4 \mathrm{~mm} \times 94.4 \mathrm{~mm} \times 98.1 \mathrm{~mm}$

- Quality: bi-linear interpolation

- Mode $=$ cone beam

- Filtering: regular

- Output type: 16bit

The resolution of the final data scan images depends on several components such as:

- the X-ray detector resolution,

- the focal spot size,

- the geometric magnification,

- the filtering algorithm used to reconstruct the images,

- the stability of the turntable on which the specimen is placed.

While dose radiation is an important factor to minimize to preserve the health of the patient being scanned, scanning a temporal bone specimen is not affected by such a factor. However, a clinical CT scanner has some presettings that cannot be overloaded by the radiological technologist. Therefore, typical scan settings were used even for the bone specimens.

A slice from a micro-CT scan of a temporal bone specimen is illustrated in Fig. 4.6. 


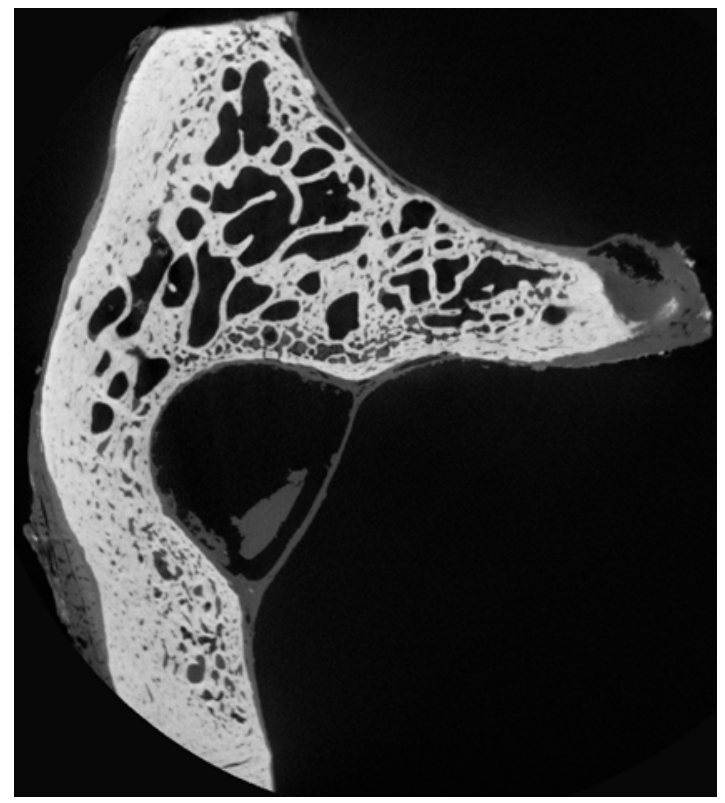

Figure 4.6: A micro-CT scan of a temporal bone specimen.

The signal-to-noise ratio (SNR), determining the quality of the scanned data from of a CT scan, depends on the total exposure. SNR is proportional to the tube current and the total exposure time. The SNR also increases with $\mathrm{kV}$ but not proportionally. In medical scanners high power is used, expressed in kilowatts, but a full scan is performed in a few seconds. In micro-CT scanning, low power is used, expressed in watts, but the specimen is exposed for a very long time instead, typically a thousand times for one second compared to a clinical CT.

\subsubsection{Estimated price}

Price is also an important parameter. A medical scanner is around 1,000,000 $€$ and possibly more. On the other hand the price of a typical micro-CT scanner can be anywhere between $50,000 €$ and 1,000,000 $€$, but 250,000 $€$ is a common average price.

The price of a single micro-CT scan is charged per hour of actual measurement time at the department of Physics and Astronomy in Ghent, and is fixed to $100 €$. For a regular clinical CT of the same temporal bone using the conventional clinical CT scan, was done for free with the agreement of acknowledgment in publications related to these scans. But an estimated cost would be similar to the fee charged for a micro-CT scan, about $100 €$. 
After presenting the equipment used to produced the data, the next chapter introduces the field of image processing, in which algorithms are used to extract information related to the surface area and volume of the mastoid air cells. The image processing tools are solely dedicated to X-ray type of image scans. Image processing is thus important for investigating the geometrical characteristics of the mastoid air cell system. 


\title{
Image Processing
}

\author{
"Abyssus abyssum invocat." \\ Deep calls to deep - (The more of the context of a problem that a scientist \\ can comprehend, the greater are his chances of finding a truly adequate \\ solution).
}

\subsection{Introduction}

This chapter provides a basic theoretical background of image processing. Common image processing tools [15], often encountered in clinical studies, as well as more advanced methods [14][26] [48] [49], more aimed towards experimental work, are introduced.

A section about volume rendering is also included in this chapter, since it relates to visualization of results from image processing along with original data.

\subsection{Basic image processing}

In this section, some basic relevant notions of image processing are given.

\subsubsection{Pixels and voxels}

As for cameras, an X-ray computed tomography imaging device converts the scanning of a body part, Fig. 5.1 to the left, into one or several digital medical images. An image is sampled using a set of 2D (ideally Dirac) impulses evenly spread across the object, see Fig. 5.1 at the centre. For each impulse function, the imaging device picks up a single light intensity, see Fig. 5.1 to the right. It should be noted that the supposedly continuous input image, as shown to the left of Fig. 5.1 is a digital image but is considered as continuous for the present explanation. 


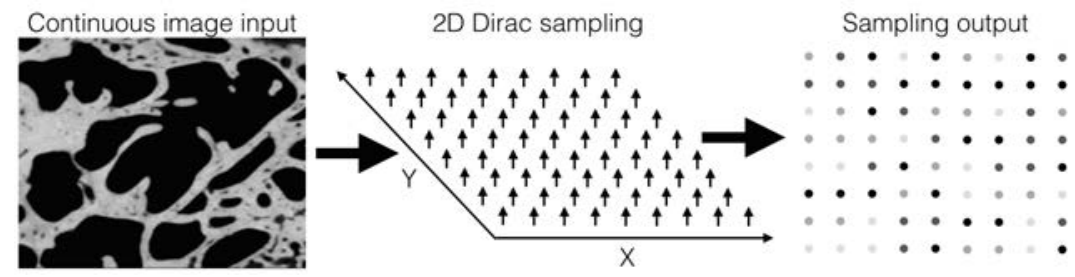

Figure 5.1: Sampling a continuous image with a field 2D (ideally Dirac) impulse functions to extract the image point-wise intensity. Note, the middle illustration is pseudo-3D representation with the plane slanted for visualizing the Dirac impulses.

To produce the final digital image, a grid (the image matrix), is virtually defined around each sample with the samples being located at the middle of each grid element, see Fig. 5.2 to the left. For visualization, the intensity value from of each sample is then spread over the corresponding grid cell through nearest neighbour interpolation, see Fig. 5.2 at the centre. This process results in picture elements, commonly known as pixels, see Fig. 5.2 to the right.

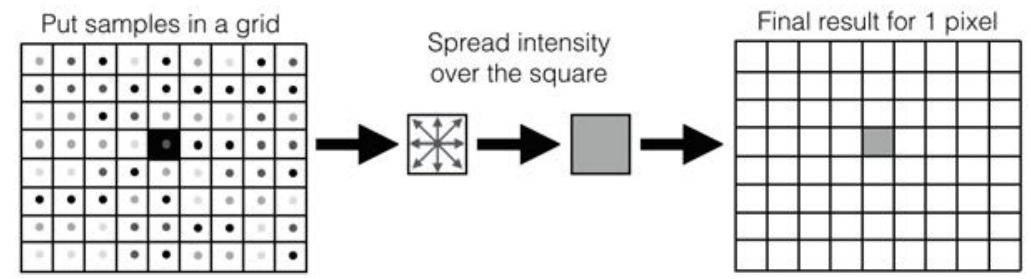

Figure 5.2: Gridding and spreading the value to the overall pixel. The black element in the left grid represents the pixel being processed, while the sampled intensity value is located in the center of the block element.

The same procedure is repeated for all samples emanating from the Dirac sampling. The final result is a digital version of the continuous element/medium being scanned, see Fig. 5.3.

The spacing between the samples defines the size of the pixel and therefore the resolution of the final digital image. A larger distance between the Dirac impulses results in a lower resolution implying a lower image quality. On the contrary, a smaller distance between the Dirac impulses results in 


\section{Digital image output}

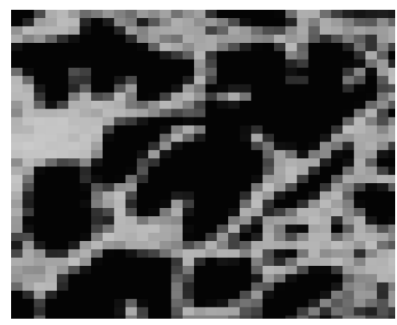

Figure 5.3: Digital output image.

a higher resolution, equivalent to a higher image quality.

In practice, a pixel represents an area rather than a single point. The value associated with the pixel is therefore best thought of as an average value over that area.

Generally, working with pixels can be done by accessing them using the notion of indices as used for matrices, where the first index denotes the columns, usually named $x$, and the second index denotes the position of the rows, usually named $y$. The x-axis indexation goes from left to right. The $\mathrm{y}$-axis indexation goes from top to bottom.

In three dimensions (3D), a pixel turns into a volume element, also known as a voxel. Using a similar approach, voxels represent an average over a volume of data. As in the $2 \mathrm{D}$ case, the voxel is represented in terms of indices, where $x$ denotes the columns, $y$ the rows, and $z$ denotes the depth.

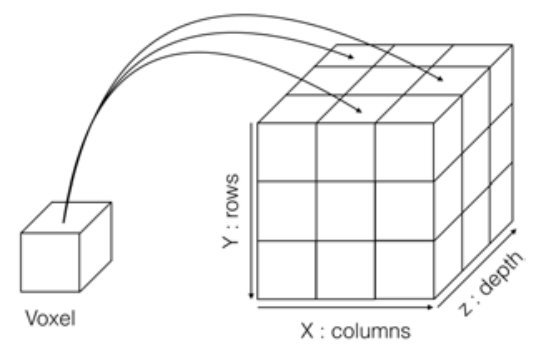

Figure 5.4: Voxel defined to the left, and 3D digital volume with the indices given.

Now that the image generation has been introduced, the content of a pixel will be briefly introduced. 


\subsubsection{Grayscale content of an image}

Medical images are most of the time stored as grayscale images representing the brightness of the structures being scanned. The resolution of the image, in terms of brightness, is defined by the number of different brightness values that are permitted. A common range of gray values is a set of 256 discrete values ranging from 0 to 255 , each pixel is then encoded as an unsigned 8-bit integer $\left(2^{8}=256\right)$. One of the main reasons for using 256 shades of grayscale values is related to the human visual system [16].

In X-ray CT scans, the lowest grayscale value, namely 0, represents pure air or liquid, while a grayscale value of 255 represents the highest density material in the image. Bone, unless metal is present, is usually the highest density material in a X-ray type of images. Soft tissue densites lie in the middle; most often more towards air than bone. A very compact representation is a so-called histogram as explained in the following.

\section{Histogram}

A histogram can be used to analyze the brightness and contrast of an image, by displaying how the grayscale values of an image are distributed over the whole range of brightness values. For each grayscale intensity present in the image, the number of pixels having this grayscale value is accumulated. The result is a graph as illustrated in Fig. 5.5, where the shaded area represents the presence of each intensity value.
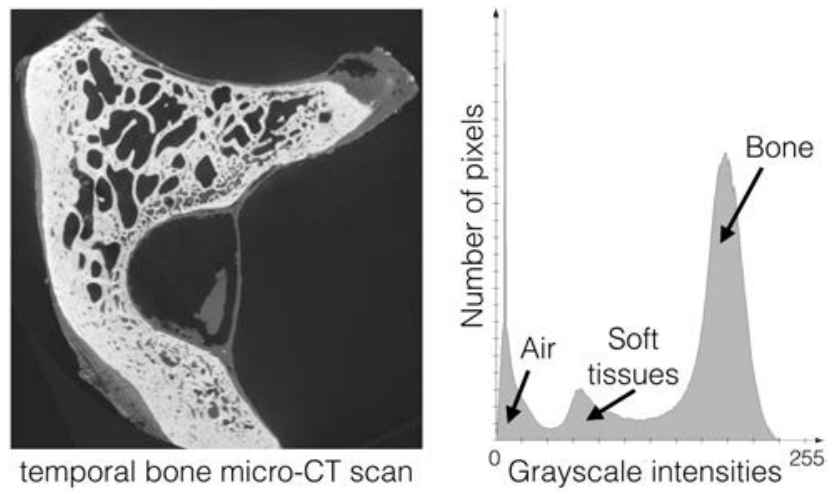

Figure 5.5: The 2D slice shown to the left has a histogram curve corresponding to the right, where each bin is an accumulated sum of all pixels belonging to each specific grayscale value.

As previously explained, three main peaks are present, see the right side of Fig. 5.5. Because the image is mostly black due to the air surrounding the 
bone, the left peak is much higher than the other two peaks. The second peak relates to the presence of soft tissues, mostly attached to the bone.

In the presence of metal, a fourth peak, very similar to the one representing air, will be visible at the very end of the histogram. The peak is normally very sharp, and is sometimes intermixed with the bone part of the histogram. The same principle applies for 3D volumes where a $1 \mathrm{D}$ histogram represents all the voxels from the data scan.

Ideally, images do not contain noise, but in reality noise is always present. In the following section, a brief discussion of noise present in the data scans used in this study is given.

\subsubsection{Image noise}

The influence of noise in the data can be quite problematic in image processing, especially when inferring statistics from the obtained measurements. There are several types of noise that can be encountered in images. A
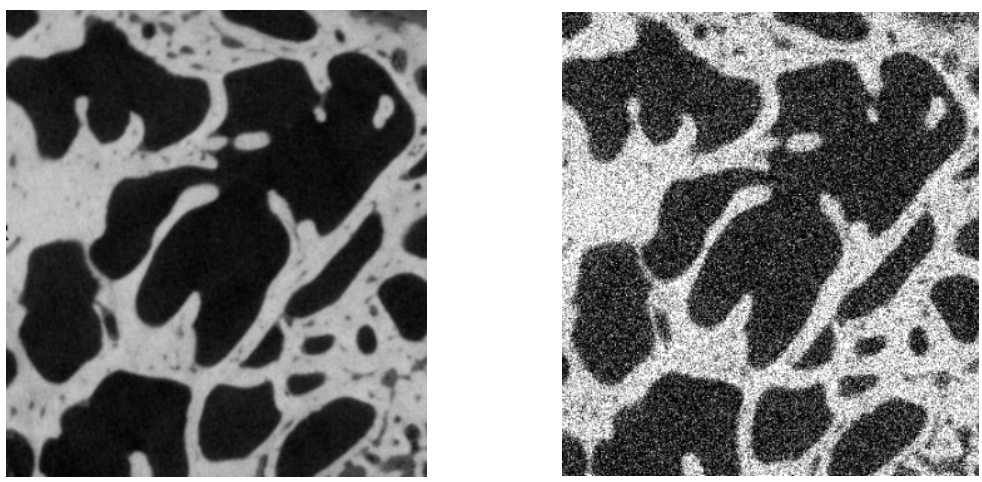

Figure 5.6: Original grayscale image (left), and noise added to the original image (Gaussian with mean: 0.5 and std dev.: 65, extreme case for clarity) (right).

common noise model in image processing is the Gaussian one. Gaussian noise is characterized by two parameters; the mean value and the standard deviation. The mean is normally zero, while the standard deviation can model the spread of the noise intensity.

A fundamental different type of signal disturbance is for instance structural noise, typically seen as rings in cone-beam CT or micro-CT scans with streak artefacts. While unstructured Gaussian noise can be filtered out in many cases, noise containing structures is much more challenging to remove. In this work, only Gaussian noise has been considered, and other types of noise have so far been discarded. 
For flat areas, where the image intensity is constant over an area or volume (e.g. air or bone), removing the noise is fairly easy. In transition areas, such as the transitions between air and bone as in the case of the mastoid air cells, the noise can be more challenging to remove. This is why it is always preferable to obtain high signal to noise ratio (SNR) data where the noise will have a minimum influence on the data to process.

Convolution can be used to filter out the noise from an image. But before explaining how to filter out the noise, a short introduction to convolution is given.

\subsubsection{Convolution}

A convolution is performed by first defining a filter (also known as mask), seen as a matrix whose values are called weights. For each pixel of the input data, the center of the filter is placed on the top of the pixel, and the pixels under the matrix are multiplied by the corresponding weights. The sum of all the multiplications is finally stored in a pixel in a new image, called the filter response. Convolution is considered as one of the most important operations for signal and image processing.

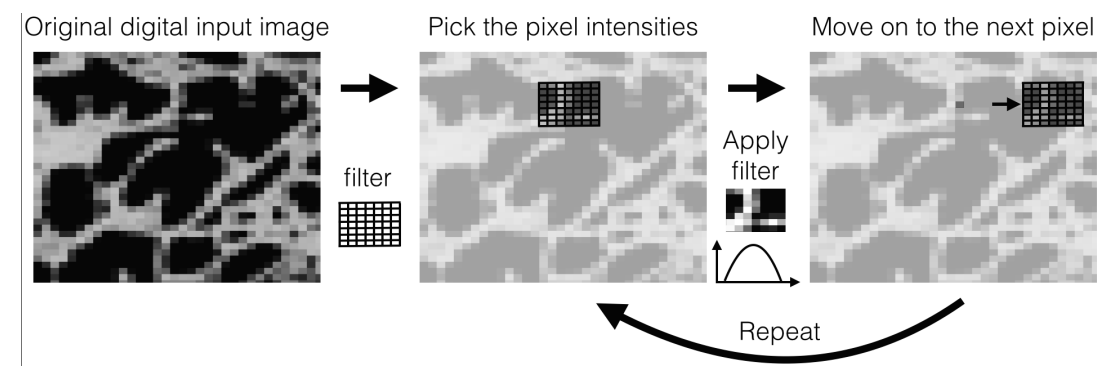

Figure 5.7: Steps involved in the convolution process: a filter is applied on the original digital image (left) to pick up a local subpart of the image at a specific pixel and the neighbouring pixels (middle), a filtering step is then locally applied on this subpart of the image, once processed, the filter is moved to the next pixel (right).

Once the convolution has been performed for all pixels, the final output is saved as a filtered image, see Fig. 5.8.

Different types of kernels or filters, such as the gradient filter to detect edges in an image can be used through the convolution operation. Gradient kernels are more specialized in obtaining the edges of objects present in the 


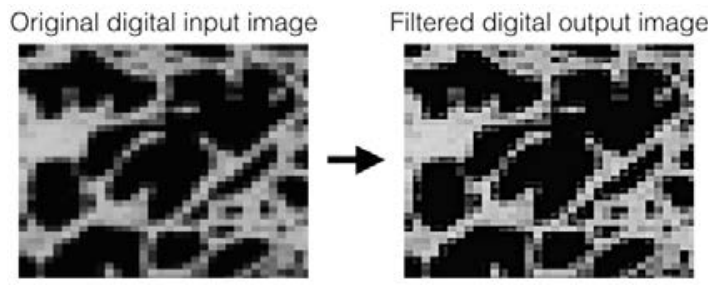

Figure 5.8: Processed digital image (right) resulting from the convolution of the original digital image (left) with a sharpening filter.

image. For 2D images, the gradient filter is applied both along the rows and along the columns, see Fig. 5.9.
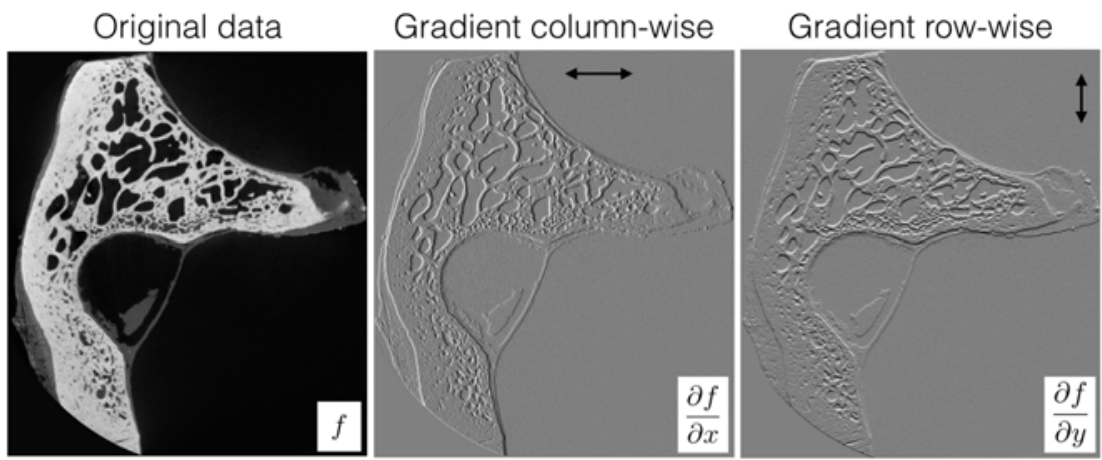

Figure 5.9: Convolution with a gradient filter, both along the $\mathrm{x}$ direction (column-wise) and along the y direction (row-wise).

When computing the magnitudes from the two gradients, along the $\mathrm{x}$ direction and along the y-direction, using the formula $\sqrt{\left(\frac{\partial f}{\partial x}\right)+\left(\frac{\partial f}{\partial y}\right)}$, the edges of the structures with a sharp transition in grayscale intensities become dominant in the output image, see Fig. 5.10. The gradient filter is also known as an edge detector.

Another type of filter is the quadrature filter, which will be introduced in the next section. All these filtering are performed through convolution of the respective kernel with the original image. In the next section, and for simplicity, a box filter is used to filter the noise from the image. Issues concerning the use of such kernels are then revealed. 

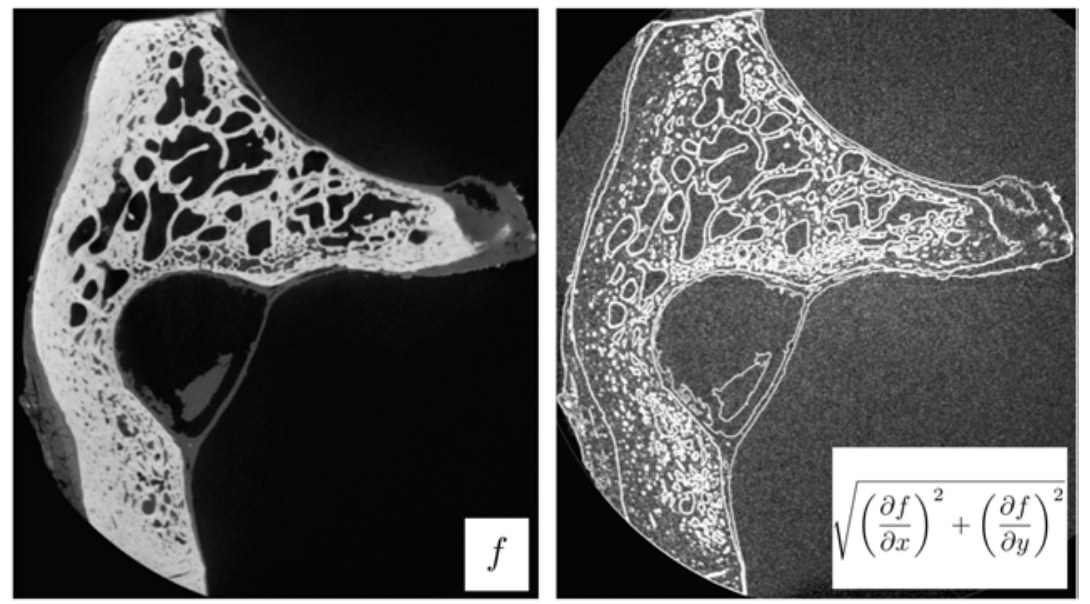

Figure 5.10: Magnitude from the gradient filtering revealing the edges of structures where sharp grayscale transitions occur.

\subsubsection{Noise filtering}

An easy way to filter the noise out is to perform a smoothing by averaging over pixels in a mask or kernel that is convolved over the noisy data. Two typical noise filters used in image processing are the box filter or the Gaussian filter, and are introduced in the following.

The box filter, also known as a mean or average filter, blurs the image and removes details as we will see part of the noise in the image as well, by simply replacing each pixel value in an image by the mean value of the neighbouring along with the pixel itself, see Fig. 5.11. A $3 \times 3$ square kernel is often used, but larger kernels, such as for instance $5 \times 5$ can be used for more severe smoothing. Note also that a small kernel can be applied successively, to produce a similar result though with some slight differences compared to a single pass when using a large kernel.
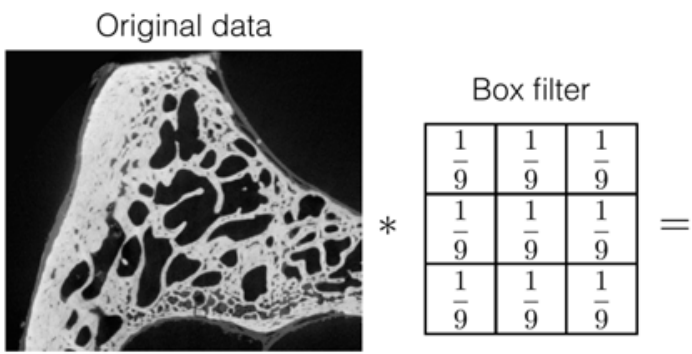

\section{Smoothed data}

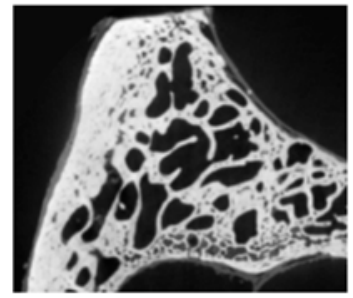

Figure 5.11: Box filter with a kernel size of $3 \times 3$. The sign $*$ means convolution. 
Another filter, commonly used, is the weighted average filter called the Gaussian filter. Gaussian filtering also blurs the image to reduce noise in the image, but the kernel is shaped as Gaussian distribution instead of a rectangular function, see Fig. 5.12. Because the image to be processed is discrete, an approximation to the Gaussian function needs to be created in a discrete fashion for the kernel, before convolving the image with the kernel.
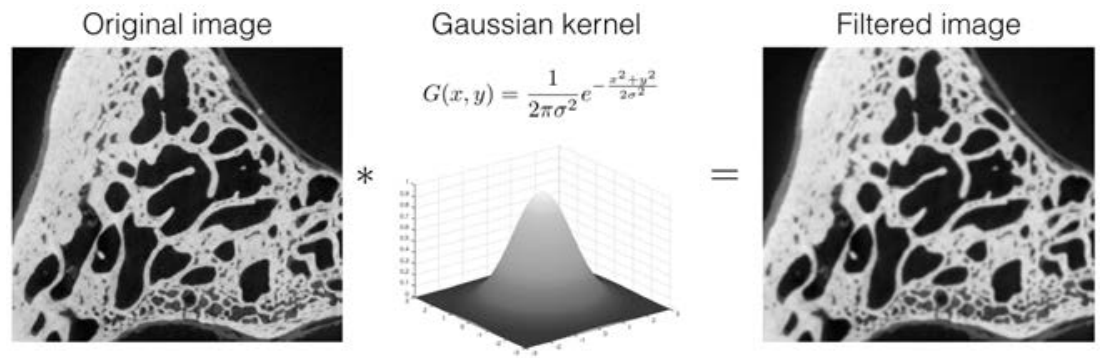

Figure 5.12: Gaussian 2D filter with a kernel size of $15 \times 15$ (middle) applied on the original image (left) to obtain a smooth version (right) of the original image. The sign $*$ means convolution.

Other image denoising techniques exist, such as bilateral filtering, non-local means, anisotropic diffusion and adaptive filtering. These techniques are detailed in [21].

Once filtered, the aim is to extract the structure of interest from the image data in order to extract metrics such as the surface area and volume. As introduced in the next section, this extraction can be done using segmentation.

\subsubsection{Segmentation by thresholding}

This section only deals with the type of segmentation used in this field of hearing research. Many other segmentation methods exists such as the local adaptive threshold, but their description is beyond the scope of this thesis and are seldomely used in the clinical field [48] [49]. The reader is invited to investigate the literature found in [21] [15].

The most common technique used in this field is to isolate a structure from the rest of the data using a technique called binary thresholding. Binary thresholding is applied in the following manner. 

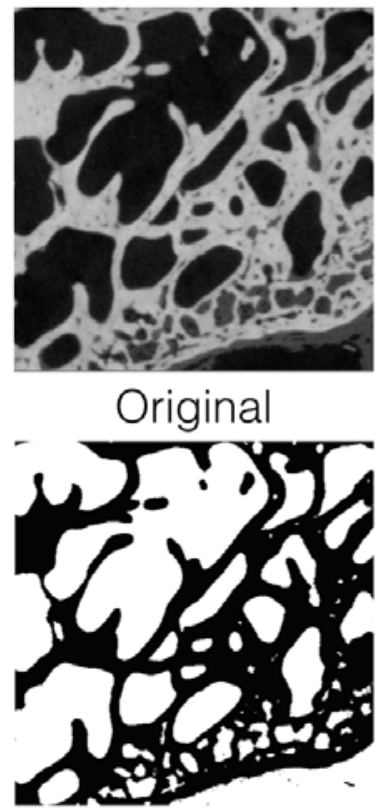

80

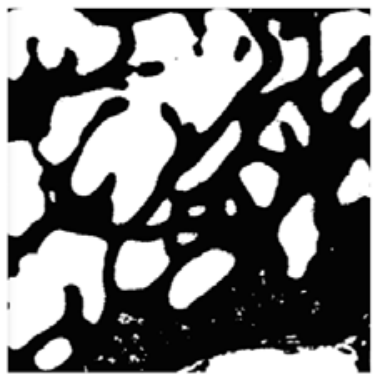

40

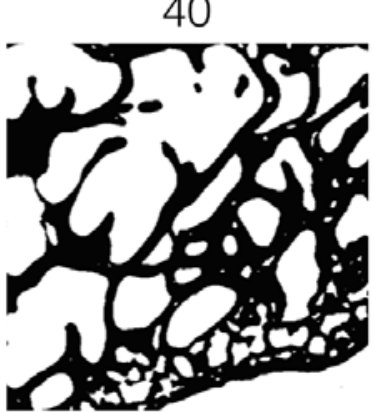

100

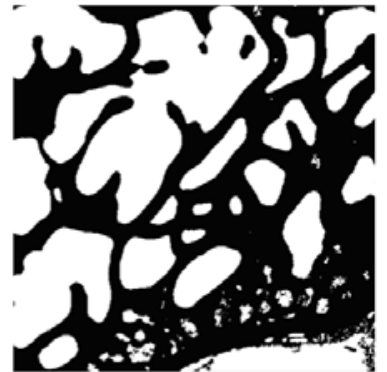

60

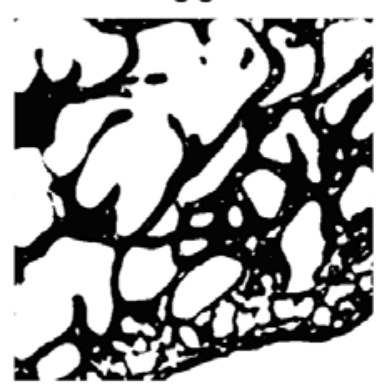

120

Figure 5.13: Binary segmentation of the airspace in the mastoid bone using different threshold values $(40,60,80,100,120)$ compared to the original data (top left).

Every pixel whose value is above a certain threshold, or within a certain range of values, is interpreted as foreground value, usually set to 1 , while all remaining pixels are considered as background, respectively set to 0 . Fig. 5.13 shows an example of an image segmented at different threshold levels. In this example, the airspaces pixels below the threshold are segmented out and represented as white in the binary mask. As can be observed, the higher the threshold the more structures are segmented along, including the bone marrow in the trabecular spaces when the threshold is above 60 . Also, it can be noticed that some of the segmented structures start to be attached to each others while they are not supposed to. This arises when the threshold is close to the bone range of grayscale values, and where the segmentation classifies some pixels belonging to bone as foreground (air) and not as background (bone). This type of error is illustrated in the simple representation shown in Fig. 5.14.

At the boundary of a structure, the CT intensity values change from the level of one tissue to that of another tissue. However, this change is gradual 


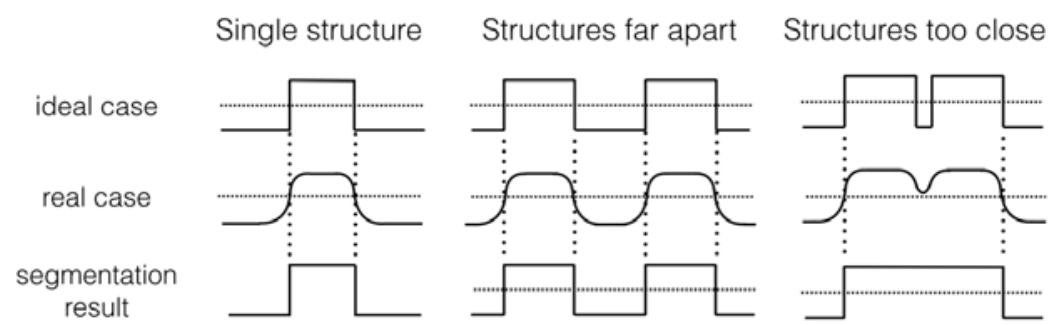

Figure 5.14: Left: single structure, middle: two structures far apart leading to the segmentation of the two structures, right: the structures are too close, which leads to a single object in the segmentation result.

rather than abrupt, giving a blurred boundary interface. The interface, in perfect condition, is measured exactly halfway between the two CT number levels, known as half maximum height, or $\mathrm{HMH}$, representing the mean value of the two CT levels from both side of the transition.

Binary segmentation does not have any a-priori knowledge of the underlying structures to be segmented. Therefore, all pixels or voxels above a certain value from different structures will be segmented at the same time, forming different components either connected or disconnected.

Because segmentation using thresholding technique does not contain information of the structure to keep, a manual selection of that structure needs to be done, see Fig. 5.15 to the right. The only requirement is to make sure that the structure is disconnected from all segmented structures. The selection was performed using a region growing tool, by first placing a seed in the structure of interest and letting the algorithm segment all voxels belonging to the structure [19].
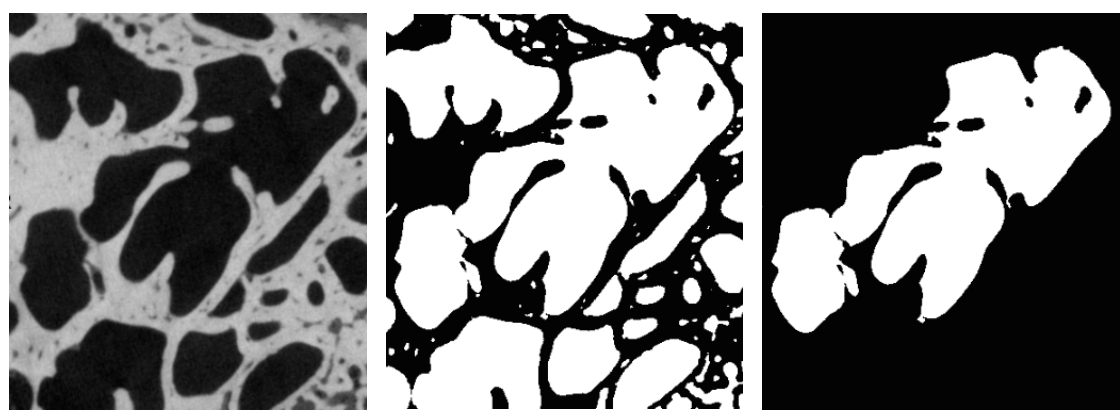

Figure 5.15: Selection of a region of interest (right) from the binary segmentation (middle) of the original data (left).

The result of the binary segmentation is often postprocessed using morphological operations, which are described in the following section. 


\subsubsection{Morphology on binary images}

Results from a binary segmentation can lead to misdetected or over-segmented regions, while the remaining parts appear acceptable. An attempt to circumvent these issues is through the use of morphological operations. Four morphological operators are commonly used: dilation, erosion, opening, and closing.

Dilation consists primarily of enlarging the boundary of all the segmented regions in the data, see Fig. 5.16.
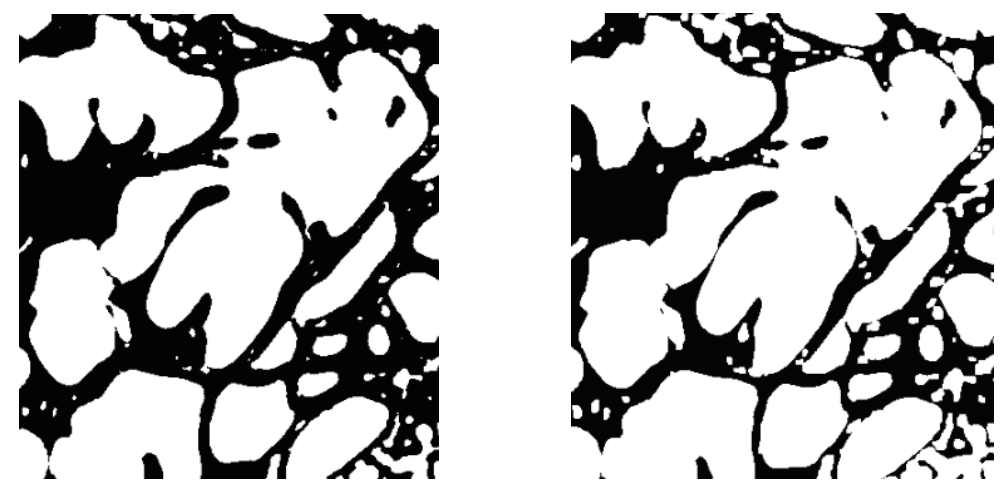

Figure 5.16: Results from dilation operation (right) compared to the original binary image (left).

Erosion, on the contrary, consists of shrinking the boundary of all the segmented regions in the data, see Fig. 5.17.
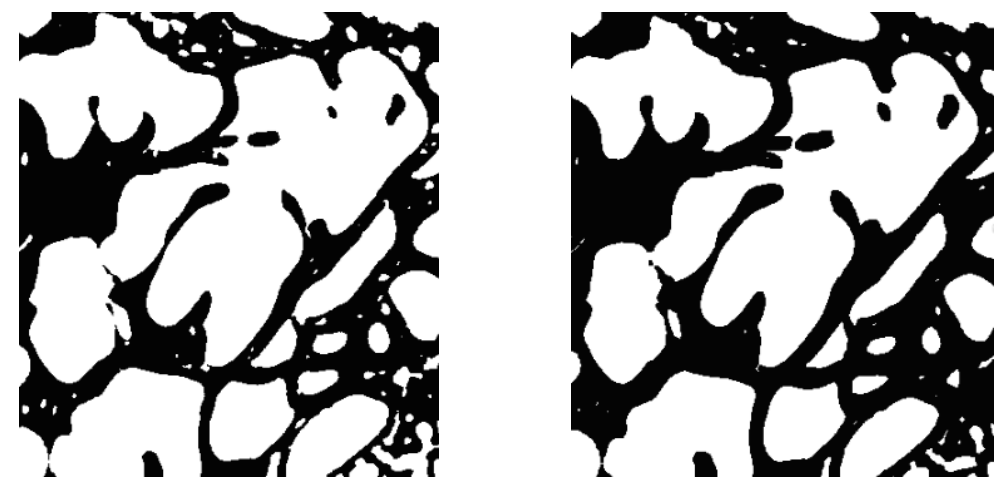

Figure 5.17: Results from the erosion operation (right) compared to the original binary image (left).

The last two morphological operators, namely opening and closing, are in fact combination of dilation and erosion in different orders. 
Opening, corresponding to a succession of erosion followed by dilation, can be seen as a morphological noise filtering. The small structures are first removed from the binary data, and then all shrunk boundaries from the erosion part are restored back to their original shape by the dilation step. An example of applying a morphological opening on binary data is shown in Fig. 5.18.
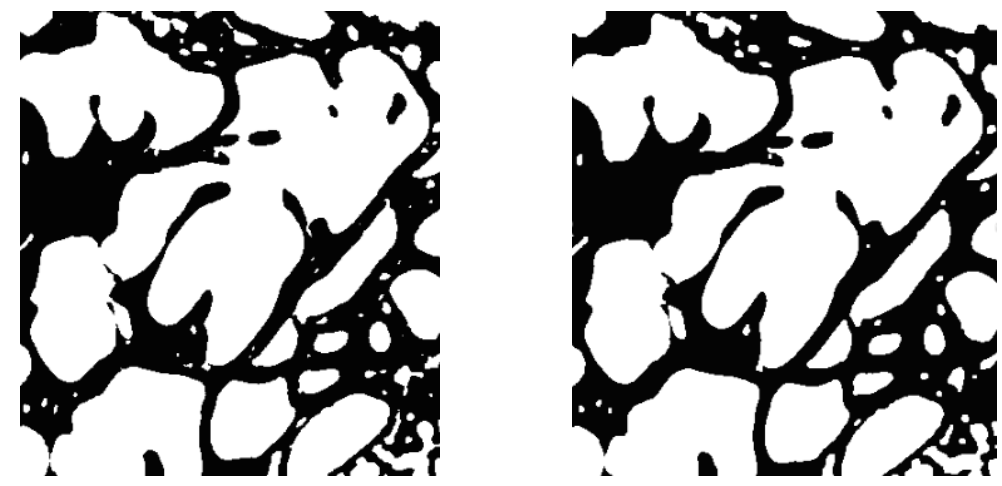

Figure 5.18: Opening operation compared to the original binary image.

Closing is, on the other hand, a succession of dilation and erosion. While opening removes noise in the binary data, closing can be interpreted as a filling the small cavities present inside a binary structure. An Example of applying a closing operation on a binary image is given in Fig. 5.19.
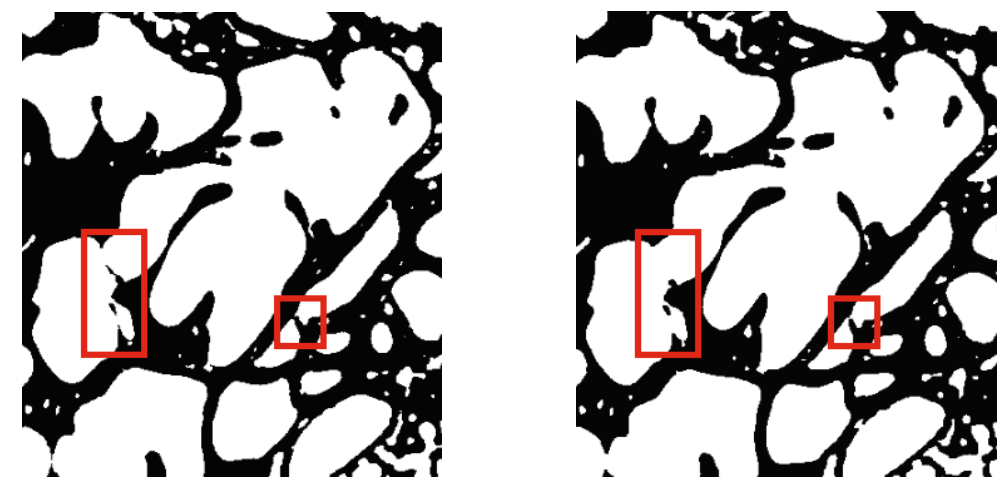

Figure 5.19: Closing operation compared to the original binary image, see the gray squares where merging occurred.

Despite a simple implementation and its usefulness for very simple shapes, such as a cube or a sphere in $3 \mathrm{D}$, results from morphological operations are very often unsatisfactory for natural images (i.e. medical digital scans).

In some cases, even after a single pass of dilation or closing, some unwanted 
holes reside in the segmented object. A hole can be defined as a region of pixels only having a background value (0), surrounded by a border of pixels with foreground value (1). The algorithm, in that case, iteratively changes the connected background pixels to foreground pixels and stops when the boundaries defining the hole of the segmented object are filled, see Fig. 5.20 .

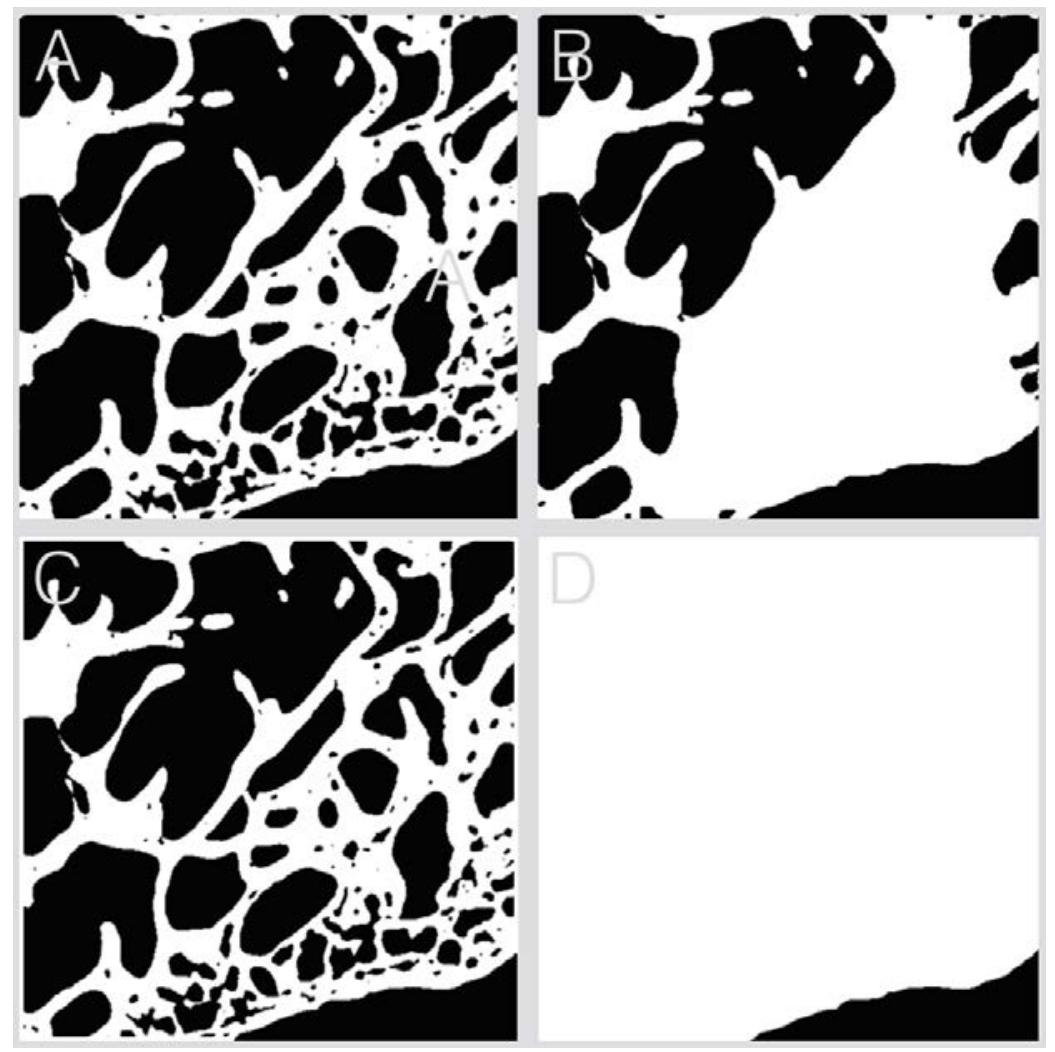

Figure 5.20: A (top left): binary segmentation of the bone, B (top right): results from the hole filling where some holes were not processed due to incomplete borders (sides of the image), C (bottom left): Modified version of $\mathrm{A}$ with the left, top, and right sides of the image covered with a foreground value line. C (bottom right): successful filling of the holes in the bone.

The cavities present in the bone in Fig. 5.20(A), when fully surrounded by foreground value, are fully covered with foreground value, see Fig. 5.20(B). However, it can be noticed that some cavities were not filled due to the boundary not fully surrounding the cavity. To illustrate the fact, vertical 
and horizontal lines were added to the side of the image where bone is present, see Fig. 5.20(A). This step ensures that all cavities would be fully be surrounded by a boundary, see Fig. 5.20(C). Only the lower right part of the image was not covered with a foreground line. When applying the hole filling algorithm on Fig. 5.20(C), all cavities inside the bone are then successively fully filled, see Fig. 5.20(D).

Such a technique can further help to extract a structure, such as the bone, with all its content. However, when looking at the binary results, it is almost impossible to know what lies under the voxels added during the morphological step(s) being used. A possible way to verify what information lies under these added pixels/voxels is to reassign the original data over the binary data, as explained in the following through the masking operation.

\subsubsection{Masking original data over a binary segmentation}

A possible way to recover the original data information from binary segmented data, is to simply multiply the binary data by the original data. All pixels or voxels that have a zero label will remain 0 , while all the other pixels or voxels that are of label 1 from the binary image data will recover the original grayscale image.
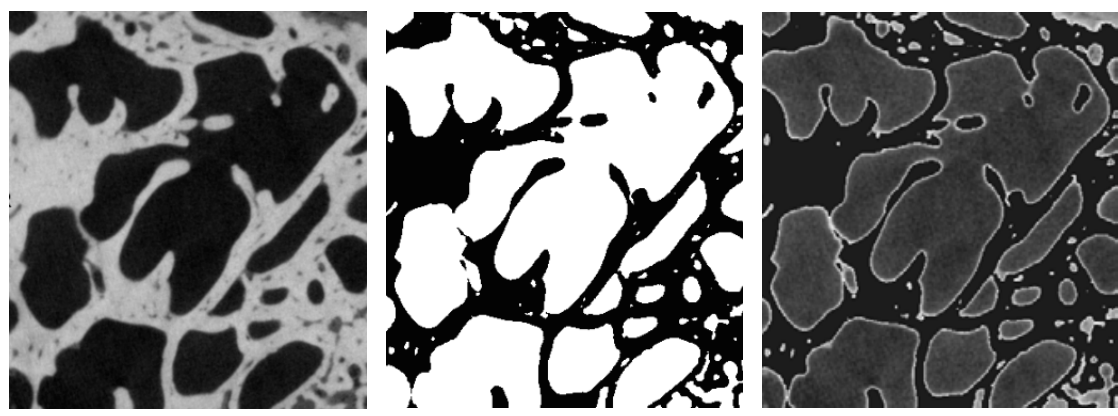

Figure 5.21: Reapplying the original grayscale data on the binary data.

In an image processing pipeline for visualization, such a method could be be relevant. However, when used as an intermediate step, masking from a binary mask may introduce sharp edges on the side of the segmented structures and can further lead to extra errors, and can worsen the results further when drawing statistics from such operations.

It may sometimes become a challenge to fix problems either related to the original image data itself, or resulting from one image processing operation. Eventually, it may become impossible to know where the problem comes from. For instance, changing the threshold in the binary segmentation may 
result in using an iteration of morphological operators, in order to allow the hole filling to work appropriately. Another example arises when seeking the true boundary of a bone. The CT level corresponding to the half maximum height should be measured locally. This is the reason why it is difficult to decide a global threshold to the overall image.

Global thresholding techniques, such as the one introduced by Otsu ([40]), which is commonly used in clinical research, are very fast and generally produce good results for the sole purpose of visualization. However, when the segmentation is aimed at measuring some metrics accurately, global thresholding will not be adequate. Local thresholding techniques estimate a different threshold for each pixel, using the grayscale information from the neighbouring pixels. However, local thresholding is rarely used in clinical research, possibly due the need for replicability of their study in relation to others or simply by not knowing its existence.

Also, an early stage segmentation discards a lot of information from the original data that could be used in further processing. A good practice is to perform a segmentation at the very last stage, where all information from the data needed for the study have been taken into account.

The mastoid air cell system being now isolated from all other structures from the scan data, metrics such as surface area and volume can be estimated, as explained in the following.

\subsubsection{Measuring surface area and volume}

Estimating the volume from $3 \mathrm{D}$ data is fairly straightforward. It is usually done through counting the number of voxels belonging to the structure, and multiply this number by the voxel size.

Voxels containing a mixture of several tissue types within the same voxel, also known as the partial volume effect, also need to be included since they contain of portion of air in them. A solution is to reapply the original data on the segmented binary data through the masking operation as explained previously. The result from the masked data is then inverted, so that it can be used as a percentage representation of the content of air in the mastoid air cells.

When the voxels are fully containing air (white), it will represent $100 \%$ of the voxel volume. Additionally, when a voxel contains a mixture of tissues, the volume of air in that voxel will provide a percentage proportional to the amount of air in it. As an example, if a voxel contains $50 \%$ of both air and soft tissue, the volume of air will be 0.5 of the voxel volume corresponding to air. 
This method produces a slightly more accurate volume estimate, than just using the binary segmented data. It should be noted that the presence of noise, as in in Fig. 5.21, can also have an influence on the volume measurement using such a technique, but is still believed to be a more accurate measure than estimating the volume from the binary segmentation.

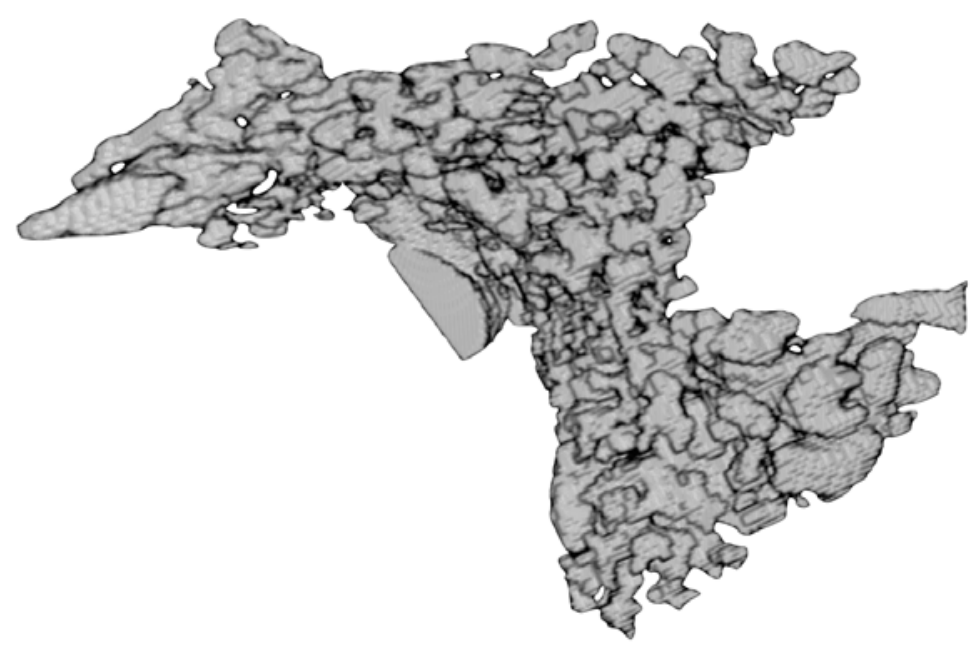

Figure 5.22: Surface mesh of a complete mastoid air cell system generated from regular clinical CT scans $(512 \times 512$ pixels with a slice thickness of $0.625 \mathrm{~mm})$.

The surface area, however, is more challenging to estimate. One technique, commonly used in many medical image processing applications, is to fit a triangular mesh from a binary segmentation [28], from which it is possible to measure the surface area. For each triangle composing the mesh, the surface area is computed and the sum of each triangle surface area is then accumulated to give the total surface area over the whole mesh.

Using such a triangular mesh, fitted to a binary segmented volume, depends heavily on the resolution of the original data. If the resolution is too low, a triangular mesh will be composed of relatively large triangles and will give a rough estimate of the surface area. When the original data is of much higher resolution, the triangles composing the mesh will be much smaller, thereby giving a much more accurate estimate of the surface area.

All the mentioned methods are based on the grayscale values of the original data. As introduced earlier, noise will inevitably corrupt the grayscale 
intensities and will inevitably lead to using different "tricks" to circumvent some of the encountered issues.

Moreover, it may be difficult to define a specific order on how to apply the different image processing tools. Nonetheless, it is often necessary to assess the results from one step, go back to a previous step, re-tune the parameters for that step, and move on to the following steps, leading to a hit-or-miss type of global method. While some methods can be used globally for the whole image, some require local adaptivity.

The image, itself, can allow the use of global methods or involve more complex local methods. Further, working with large dataset implies a lot of bookkeeping, especially when working with blocks of data to be combined again afterwards.

Also, it can be noted that this line of thinking leads to segmenting the structure of interest in order to extract information from it. Another strategy is to define the conditions for the structures of interest to be investigated, and use such a-priori information in order to extract these structures from the original input data. The next section introduces more advanced image processing methods, aimed towards the implementation of such a philosophy.

\subsection{More advanced image processing}

In this section, a different line of thinking is presented. It relates to extracting all relevant a-priori information about the structure of interest and then use this information to guide the algorithm to detect these structures of interest. Before defining the conditions to find such a-priori information, several topics need to be introduced to understand this line of thinking. For humans, as for many other species, vision is a very important part of our sensory system. Detecting a special object of interest in the surrounding involves different sources of information such as for instance colours, patterns, and shapes.

In fact, an object is mostly characterized by its size and its edges, whether being sharp or smooth. Lines are also important in the vision system, since they allow us to delineate between different structures or objects. Using such information is therefore essential when looking for a specific structure in a $2 \mathrm{D}$ image or $3 \mathrm{D}$ volume.

Even if an object of interest has globally a very complex structure, it can locally be represented with very simple structures such as lines or edges, 


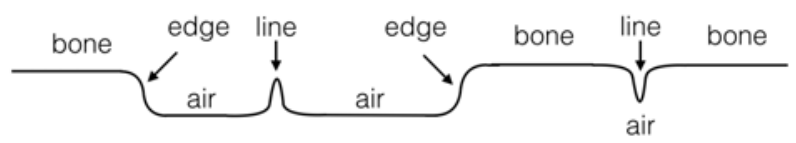

Figure 5.23: Examples of lines and edges.

all with different orientations, see Fig. 5.23. A real case is shown in Fig. 5.24 .

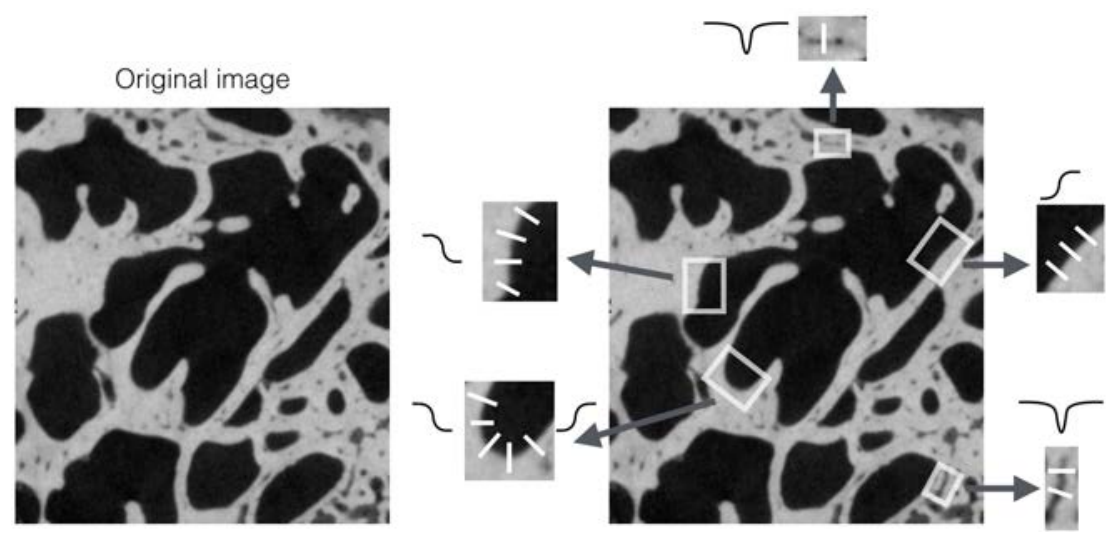

Figure 5.24: Lines and edges in different regions of an image.

Such transitions, whether a line or an edge, can be seen as simple signals in 1D. One approach is to develop a method defining a one-dimensional template for a line and for an edge, rotate these templates in several directions ( $2 \mathrm{D}$ or $3 \mathrm{D})$, and detect them in the original $2 \mathrm{D}$ image or $3 \mathrm{D}$ volume. Relevant tools from digital signal processing can be used when building such filters [25]. As will be explained in the following of the thesis, information about the frequency domain along with the spatial domain is needed.

In fact any signal, such as a complete image, can be decomposed in terms of sinusoids with different frequencies. For instance, if a profile is taken along a single image from a data scan, the grayscale intensity profile along that line can be extracted, see Fig. 5.25. It should be noted that a line in $2 \mathrm{D}$ corresponds to a sheet or a surface in $3 \mathrm{D}$.

According to Fourier theory, the signal describing the profile can be decomposed into sinusoids each having a unique frequency, see Fig. 5.26. When combining all these sinusoids with respective frequencies, it is possible to 


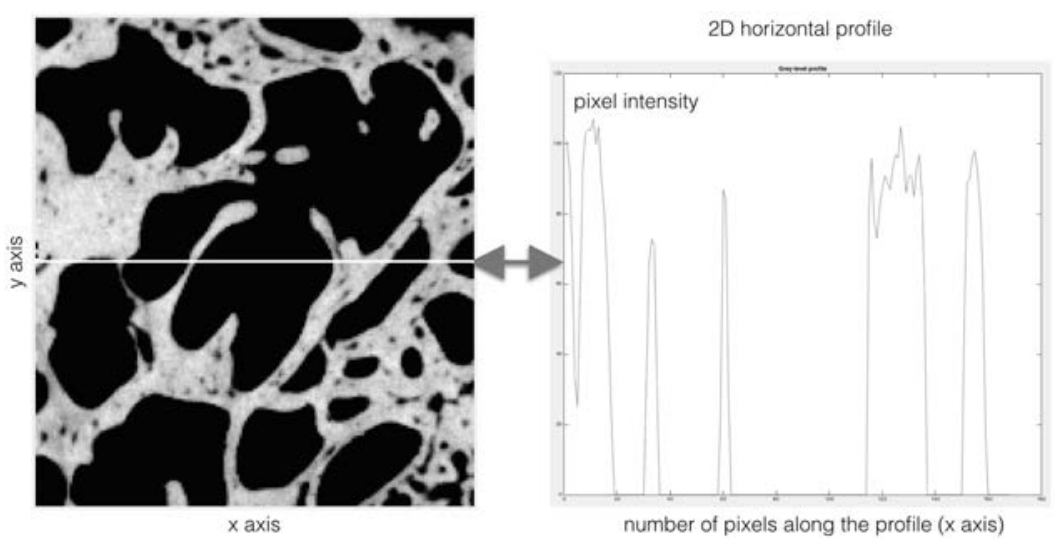

Figure 5.25: Grayscale intensity profile (right) extracted from the white line overlaid on the original image (left).

reconstruct the original profile shown in Fig. 5.25. In this example, the reconstruction from three different set of frequencies are shown. It can be observed that the reconstructed profile in the lower graph is very similar to the intensity profile shown to the right in Fig. 5.25.

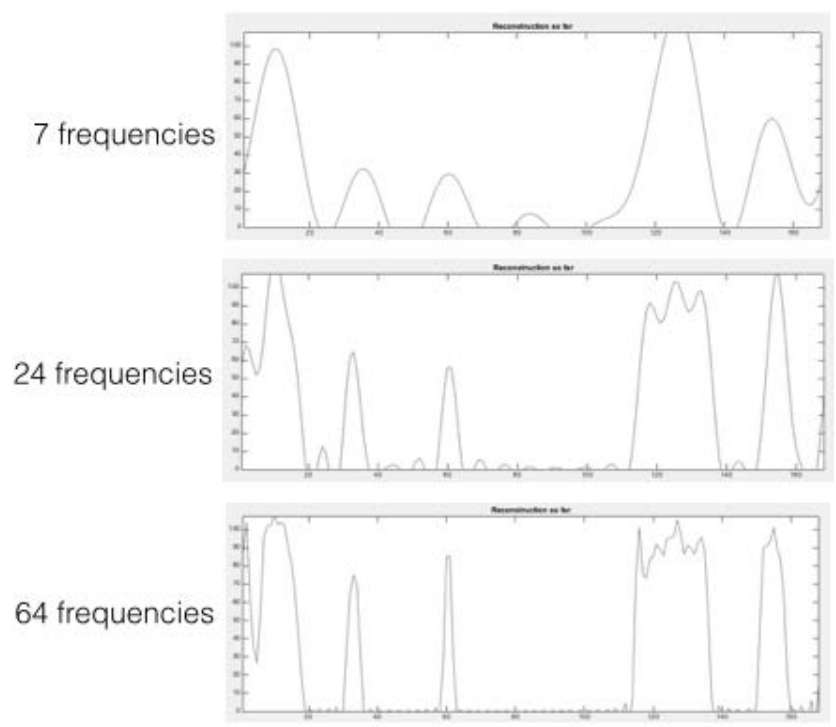

Figure 5.26: Reconstruction of the intensity profile given in Fig. 5.25 from three different sets of sinusoids.

The operation of breaking this intensity profile into sinusoids of different fre- 
quency was performed through a so-called Fourier transform. The Fourier transform plays a central role in signal and image processing, and is used when defining the template for line and edge detection in this study.

In the following section, all elements related to the design of the line and edge filters are briefly introduced focusing more on the philosophy rather than on the mathematical aspect, though some mathematical expressions will be given. A more complete account is given in [16].

\subsubsection{Filter design}

As introduced earlier, the edges and lines defining the structures can be decomposed in terms of sines and cosines. To pick up sine and cosine information from a natural signal, a solution is to create a filter to only pick a certain wavelength or range of frequencies. For illustrative purposes, only one frequency is shown in the following figures.

Before digging into the filter design, the Fourier transform of both the cosine and sine needs to be resumed first. For the cosine, defined as $f(t)=\cos (\omega t)$ with $\omega=2 \pi f$ for a $1 \mathrm{D}$ signal in the spatial domain, the Fourier transform becomes

$$
\begin{aligned}
F(u) & =\int_{-\infty}^{\infty} f(t) e^{-i 2 \pi u t} d t \\
& =\int_{-\infty}^{\infty} \cos (\omega t) e^{-i 2 \pi u t} d t \\
& =\int_{-\infty}^{\infty} \cos (\omega t)[\cos (-2 \pi u t)+i \sin (-2 \pi u t)] d t \\
& \left.=\int_{-\infty}^{\infty} \cos (\omega t) \cos (-2 \pi u t) d t+i \int_{-\infty}^{\infty} \cos (\omega t) \sin (-2 \pi u t)\right] d t \\
& =\underbrace{\int_{-\infty}^{\infty} \cos (\omega t) \cos (-2 \pi u t) d t}_{-\infty}-i \int_{-\infty}^{\infty} \cos (\omega t) \sin (2 \pi u t)] d t \\
& =\frac{1}{2}[\delta(u+w)+\delta(u-w)]
\end{aligned}
$$

The Fourier transform of a cosine is illustrated in Fig. 5.27. Where two impulse responses are located at $-\omega$ and $+\omega$.

Similarly for the sine wave, defined as $f(t)=\sin (\omega t)$ with $\omega=2 \pi f$ in $1 \mathrm{D}$ in the spatial domain, the Fourier transform becomes:

$$
F(u)=\frac{1}{2} i[\delta(u+\omega)-\delta(u-\omega)]
$$

As can observed, the Fourier transform of the sine is imaginary, see Fig.5.28. 

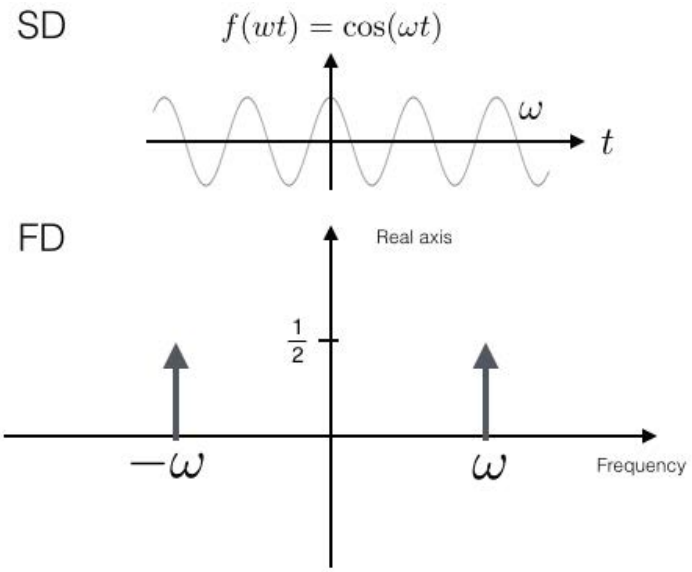

Figure 5.27: Fourier transform of a cosine.

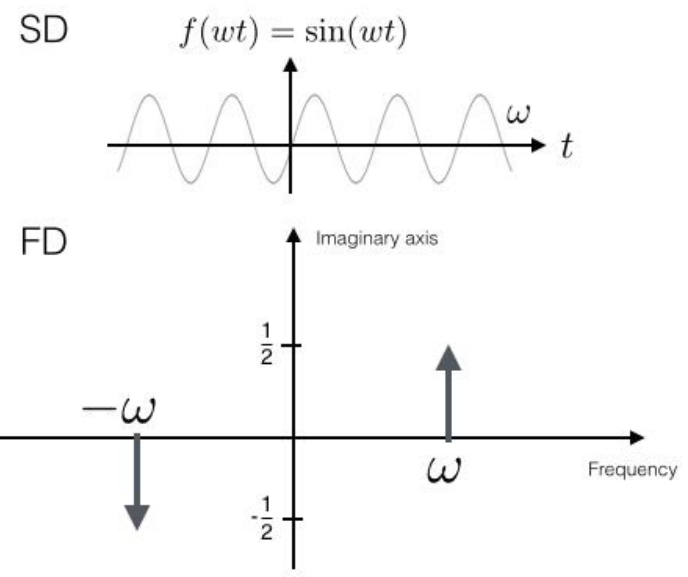

Figure 5.28: Fourier transform of a sine.

Fig. 5.29 shows side by side, using three axes, the Fourier transform of $\cos (w \mathrm{t})$ and $0 \sin (w t)$. The Fourier transform of the cosine lies along the plane formed by the real axis and the frequency axis. The Fourier transform of the sine lies on the plane formed by the imaginary axis and the frequency axis, normal to the real plane.

To avoid involving complex notation, a possible solution is to perform the Fourier transform of $i \sin (\omega t)$ instead of $\sin (\omega t)$, leading to a rotation of the Fourier transform of the sine along the real plane instead of the imaginary plane, see Fig. 5.30 or in a simpler form viewed in 2D form as in Fig. 5.31. 

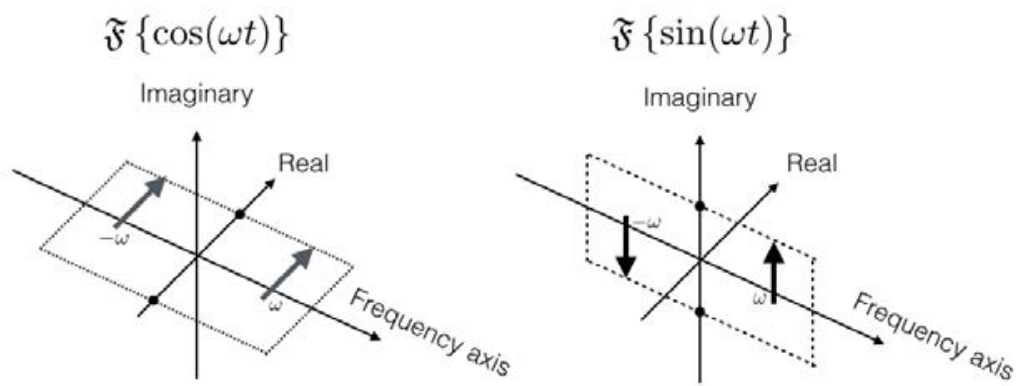

Figure 5.29: Fourier transform of cosine located along the real plane and the Fourier transform of the sine is located along the imaginary plane, normal to the real plane.
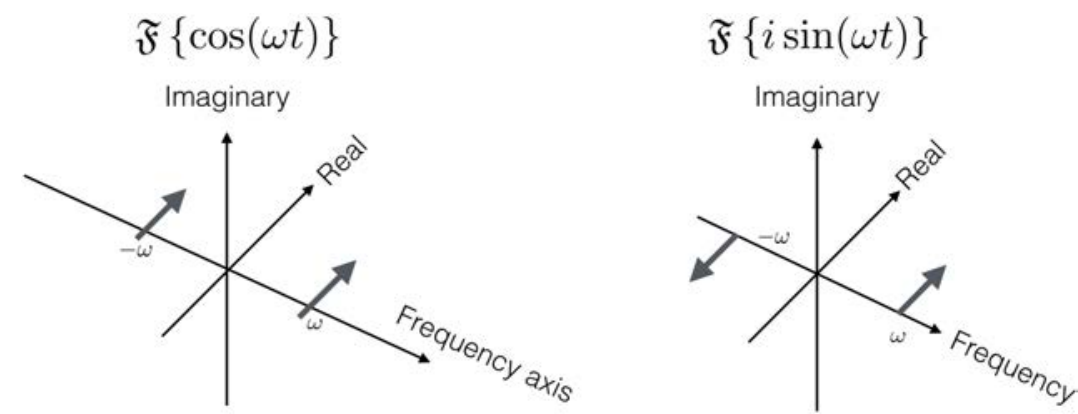

Figure 5.30: Fourier transform of cosine and the sine are now both lying located along the real plane.

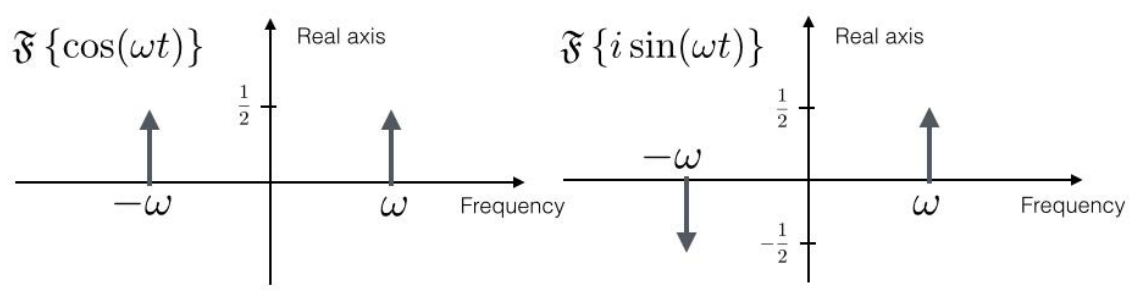

Figure 5.31: Fourier transform of cosine and the sine are now both lying located along the real plane.

Obviously, since the Fourier transform of a cosine and a sine each produce only a set of impulses for both $\omega$ and $-\omega$ whether positive or negative, using such impulse response to design a filter would make no sense since their inverse Fourier transform would have an infinite support. A filter by 
definition needs to be local in order to extract local information from an image.

To achieve locality, setting an impulse for different frequencies very close to each other around a frequency of interest, the centre frequency, and adding the contribution of each impulse will produce a filter that becomes more and more local. As an example, let's take a cosine and a sine for 5 different frequencies, see Fig. 5.32.
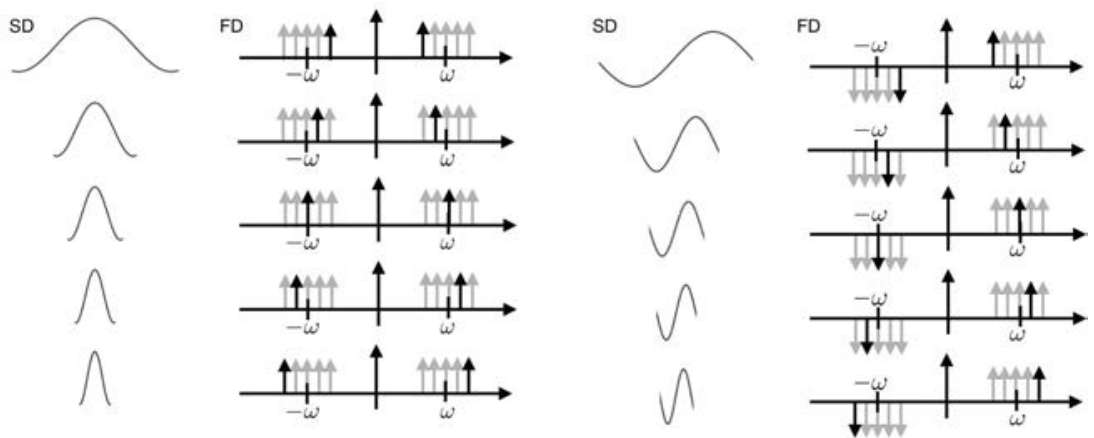

Figure 5.32: 5 cosines (left) and sines (right) with the same respective frequencies, represented both in the spatial domain and in the frequency domain. Only one period is represented for clarity purpose, but each impulse in the frequency domain represents a infinite long cosine or sine of the specified frequency.

When adding cosines with different frequencies together, a major signal component appears at the centre with some ripples on either side of the component, see Fig. 5.33 (left column). The same applies for the sine components added together, Fig. 5.33 (right column).

The quest of localization for the filter is then to suppress the ripples on either side of the signals, while keeping the major component. To give more emphasis on the main lobes at the centre of the signals in Fig. 5.33, while attempting to discard the ripples, a weighting window or function can be applied to the impulses used during the summation. In Fig. 5.34, two different types of weighting are applied.

A first weighting function is made linear corresponding to a pyramid, with for instance a weight of 0.1 for the outmost frequency in relation to $\omega$ (respectively $-\omega$ ), a weight of 0.5 is given to the innermost impulse, while the middle impulse, $\omega$ or $-\omega$, is given a weight of 1 . In the second part a non-linear weighting function is used instead of a linear one, with for this 

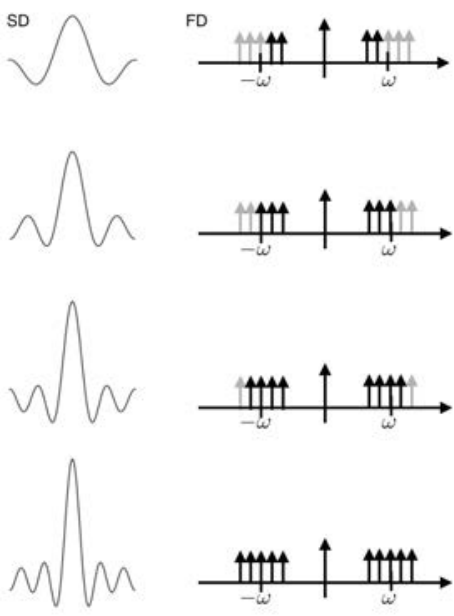
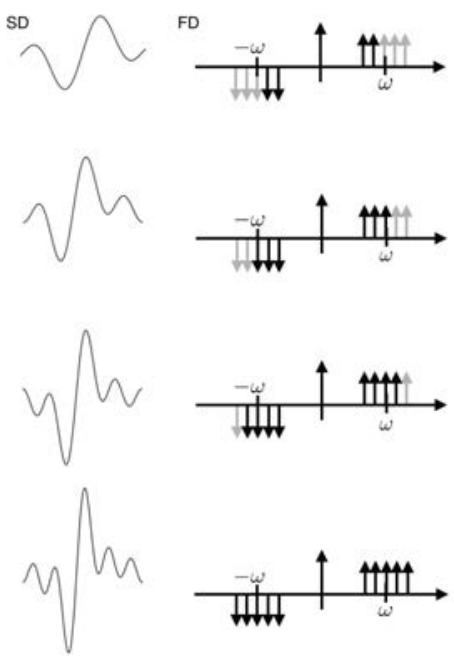

Figure 5.33: Summation over 5 frequencies in the spatial domain for both the cosine (left) and sine (right) and representation of the impulses that were used during the summation.

example a weight of $0.2,0.7,1,0.7$, and 0.2 respectively.

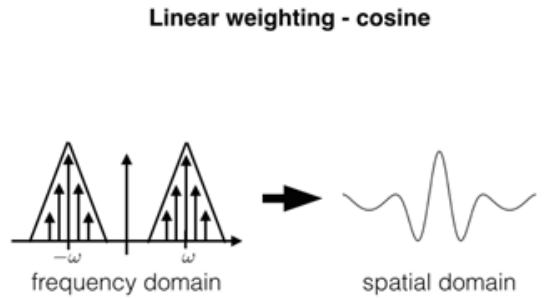

Nonlinear weighting - cosine

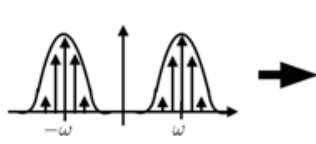

frequency domain

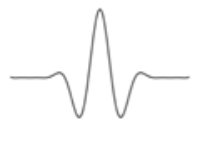

spatial domain
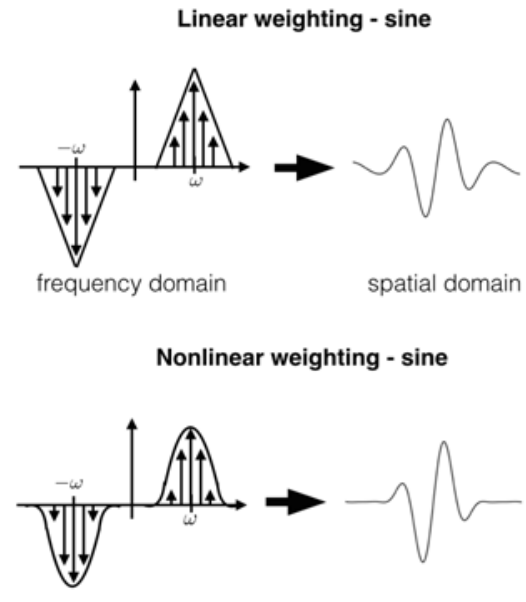

frequency domain

Figure 5.34: Linear and non-linear weighting functions applied to the impulses before summation, giving the effect on the signals in the spatial domain. 
The ripples, in the case of non-linear weighting appear to be minimized, making the filter in the spatial domain very localized. Overlapping the resulting signals from the non-linear weighting - i.e. cosine and sine - in the spatial domain, we can observe a good match in localization, see Fig. 5.35 .

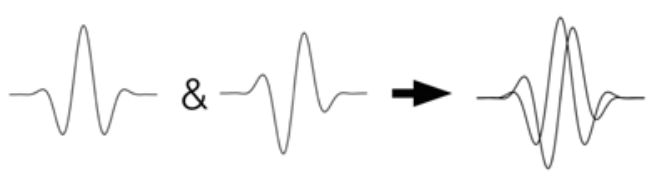

Figure 5.35: Overlapping the results from the non-linear weighting of both the cosine and sine.

From Fig. 5.35, the left signal is even in relation to its center and is defined in the real part as illustrated in Fig. 5.29 to the left. The second signal to the left is odd in relation to its center, and is defined in the imaginary part as illustrated in Fig. 5.29 to the right.

If the even part is denoted $f_{e}(x)$ and the odd part denoted $f_{o}(x)$, we can see that $f_{o}(x)$ is the Hilbert transform of $f_{e}(x)$. This property leads to the notion of quadrature, where these two components form a complex pair in the spatial domain. The real part is defined as a line filter and the imaginary part is defined as an edge filter.

When convolving the quadrature filter over an image, when a line structure is met, the response of the filter will be dominant in the real part, while when the filter encounters an edge, it will produce an imaginary response.

It is possible to map the type of structure encountered in the image, using the so-called local phase and local amplitude or magnitude [23]. The local phase is defined as

$$
\theta(x)=\arctan \left\{\frac{\mathrm{f}_{\mathrm{e}}(\mathrm{x}) * \mathrm{f}(\mathrm{x})}{\mathrm{f}_{\mathrm{o}}(\mathrm{x}) * \mathrm{f}(\mathrm{x})}\right\}
$$

where $*$ denotes the convolution. The local magnitude is expressed as

$$
A(x)=\sqrt{\left[f_{e}(x) * f(x)\right]^{2}+\left[f_{o}(x) * f(x)\right]^{2}}
$$

The local phase can be mapped in a color coding representation on a complex plane where $\theta$ represents the angle and $A$ represents the magnitude of the vector, illustrated in Fig. 5.36. 


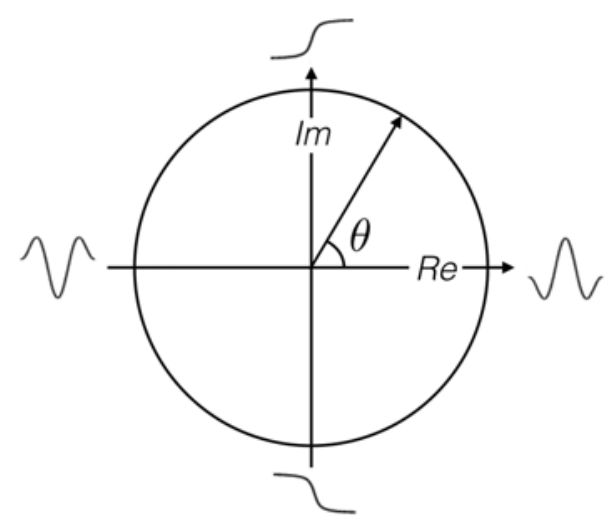

Figure 5.36: Coding of the local phase.

The advantage of using the local phase using quadrature filters is the fact that it is contrast independent. Where gradient-based type of filters can most of the time only detect edges formed by rapid transitions between two distinct regions of different intensities, the gradient-based filters will very often fail in detecting smooth edges or edges having low contrast, [26]. In the case of local phase a line, hardly detectable using common gradientbased filters, will provide the same local phase as a weak defined line. A strong magnitude provides a high confidence in the detected structure type. In locations where the magnitude is close to zero, meaning a little confidence, the local phase mainly represents noise. In Fig. 5.36, the argument of the local phase is kept constant to a maximum on the unit circle.

The quadrature filter used in this work is presented in the next section.

\subsubsection{The quadrature filter}

To pick up energy in all possible orientations, the quadrature filter is built as polar (i.e. spherically) separable in the Fourier domain with an arbitrary but positive radial bandwidth function $R(\rho)$ and a direction function $D_{k}(\hat{\mathbf{u}})$.

$$
F_{k}(\hat{\mathbf{u}})=R(\rho) D_{k}(\hat{\mathbf{u}})
$$

Mathematically, the quadrature filter in the frequency domain, $F_{k}(u)$, is defined as [23]

$$
\begin{cases}F_{k}(u)=R(\rho)\left(\hat{\mathbf{u}}^{T} \hat{\mathbf{n}}_{k}\right)^{2} & \text { if }\left(\hat{\mathbf{u}} \cdot \hat{\mathbf{n}}_{k}\right) \geq 0 \\ 0 & \text { otherwise }\end{cases}
$$

where $u$ is the coordinate vector in the Fourier domain, $\hat{u}$ is a unit vector directed along $u, \mathbf{n}^{k}$ is the direction of filter $k$, and $\rho=|u|$. 
The radial basis function and the direction function are detailed in the following subsections.

\section{The radial basis function}

The radial bandpass function $R(\rho)$ is typically designed as a bandpass function based on a center frequency and a bandwidth. A suitable radial function for estimating local image structure is based on the log normal function, a normal distribution in the logarithmic scale:

$$
R(\rho)=e^{\left(4 \ln 2 / B^{2}\right) \ln 2\left(\rho / \rho_{0}\right)}
$$

where $B$ is the relative bandwidth of the filter and $\rho_{0}$ is the center frequency of the filter, illustrated in Fig. 5.37.

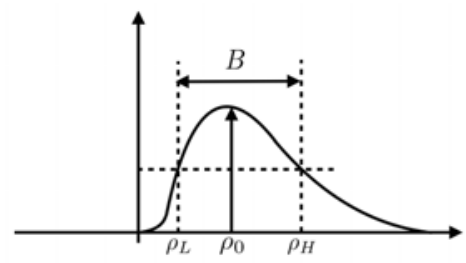

Figure 5.37: Shape of the lognormal function defined with the bandwidth $B$, the low and high cutoff frequencies $\rho_{L}$ and $\rho_{H}$ defining the centre frequency $\rho_{0}$.

It determines in what scale the filter has its sensitivity, meaning what the filter will detect. Practically, the centre frequency and the bandwidth, respectively given by:

$$
\rho_{0}=\sqrt{\rho_{\text {low }} \cdot \rho_{\text {high }}}
$$

and

$$
\beta=\frac{\log _{\mathrm{n}}\left(\frac{\rho_{\mathrm{high}}}{\rho_{\text {low }}}\right)}{\log _{\mathrm{n}} 2}(d B)
$$

with $\rho_{\text {high }}$ and $\rho_{\text {low }}$ corresponding to the high and low frequency bounds defining the bandwidth of the filter. The centre frequency corresponds to a wavelength (measured in pixels):

$$
\lambda_{0}=\frac{2 \pi}{\rho_{0}}
$$

The filter should cover at least one wavelength and in practice 1.5 or even 2 wavelengths. The following table helps choosing the spatial size: 


\begin{tabular}{ccc}
\hline centre frequency $\mu_{0}$ & wavelength $\lambda_{0}$ & linewidth $\left(\lambda_{0} / 2\right)$ \\
\hline \hline$\pi$ & 2 pixels & 1 pixels \\
$\pi / 2$ & 4 pixels & 2 pixels \\
$\pi / 4$ & 8 pixels & 4 pixels \\
\hline
\end{tabular}

\section{The direction function}

The direction function, $D_{k}(\hat{\mathbf{u}})$, varies as $\cos ^{2}(\phi)$ where $\phi$ is the difference between $\mathbf{u}$ and the filter direction $\mathbf{n}_{k}$ and given by

$$
D_{k}(\mathbf{u})= \begin{cases}\left(\hat{\mathbf{u}} \cdot \hat{\mathbf{n}}_{k}\right)^{2} & \text { if }\left(\hat{\mathbf{u}} \cdot \hat{\mathbf{n}}_{k}\right) \geq 0 \\ 0 & \text { otherwise }\end{cases}
$$

where $\hat{\mathbf{n}}_{k}$ are the directions in the Fourier space where each quadrature filter picks up energy from the local neighborhood. In $3 \mathrm{D}$, it is common to use six quadrature filters along different directions, see [16] for further explanations about this specific choice. The six directions are defined as

$$
\begin{array}{llll}
n_{1}=c & \left(\begin{array}{lll}
a & 0 & b
\end{array}\right)^{T}, \\
n_{2}=c & \left(\begin{array}{lll}
-a & 0 & b
\end{array}\right)^{T}, \\
n_{3}=c & \left(\begin{array}{lll}
b & a & 0
\end{array}\right)^{T}, \\
n_{4}=c & \left(\begin{array}{lll}
b & -a & b
\end{array}\right)^{T}, \\
n_{5}=c & \left(\begin{array}{lll}
0 & b & a
\end{array}\right)^{T}, \\
n_{6}=c & \left(\begin{array}{lll}
0 & b & -a
\end{array}\right)^{T} .
\end{array}
$$

with $a=2, b=1+\sqrt{5}$, and $c=\frac{1}{\sqrt{10+2 \sqrt{5}}}$.

The combination of the radial function and the angular function is summarized in Fig. 5.38. The radial function is by itself smooth, but to get a smooth transition between the angular function, a $\cos ^{2}$ function as a directional function.

Without the $\cos ^{2}$ weighting, the sharp transitions would result in undesired effects on the final output data. This graph illustrates the case for an image. In $3 \mathrm{D}$, a spherical shell with a thickness corresponding to the bandwidth of radial function would be formed.

A pseudo-representation of the radial function in 2D is given in Fig. 5.39 illustrating the lognormal function used as a radial function viewed from above.

The magnitude of the quadrature filter output is then computed as

$$
q_{k}=\left\|\frac{1}{2 \pi} \int S(\mathbf{u}) F_{k}(\mathbf{u}) d \mathbf{u}\right\|
$$




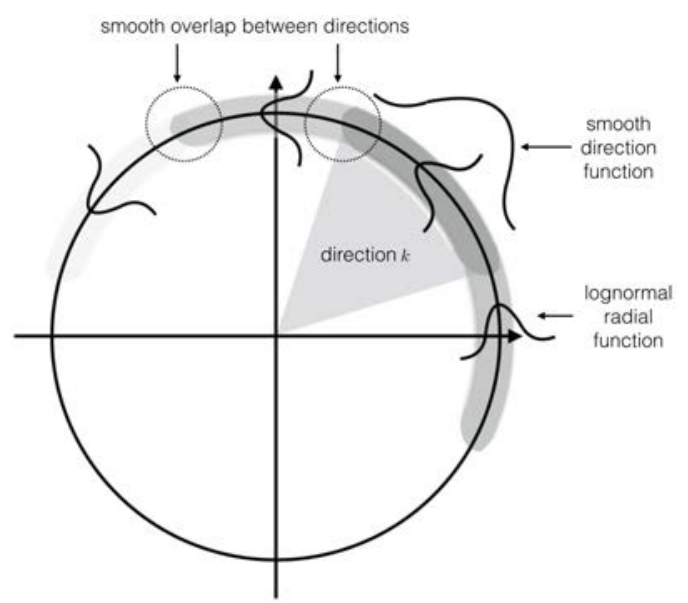

Figure 5.38: Sketch of the radial and angular functions for different directions $k$ with their respective smooth variations.

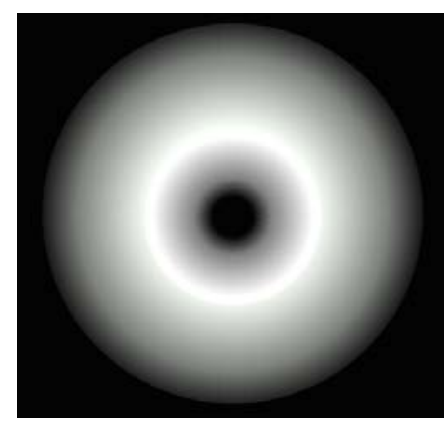

Figure 5.39: Pseudo-representation of the lognormal radial function in 2D.

where $S(\mathbf{u})$ is the Fourier transform of the original data set.

Because both spatial and frequency domains are used at the same time, the ideal filter needs to be fitted into a limited mask (both in the spatial and in the frequency domains), an operation called filter optimisation is needed [24] [25].

An example of the real and imaginary parts of an optimised quadrature filter are represented in Fig. 5.40, and the corresponding optimized quadrature filter in the frequency domain is illustrated in Fig. 5.41.

For each local neighborhood of each visited pixel, the filter response provides a contribution from the six directions. The contribution of the filter response from each direction of detected local structure needs to be stored, for each visited voxel in the image. Such a contribution cannot be rep- 

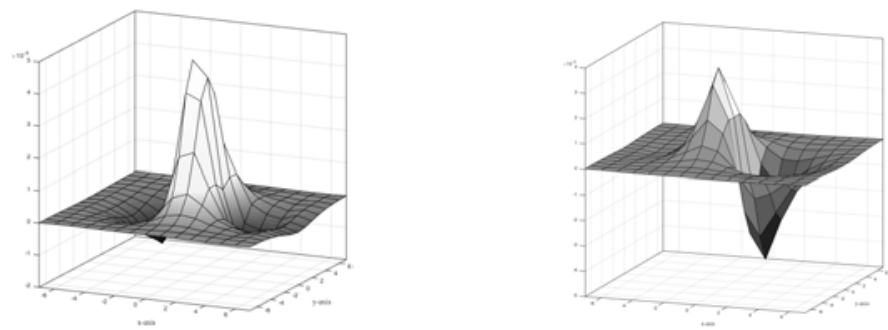

Figure 5.40: Real and imaginary part of the optimized quadrature filter: left) real part, b) imaginary part.

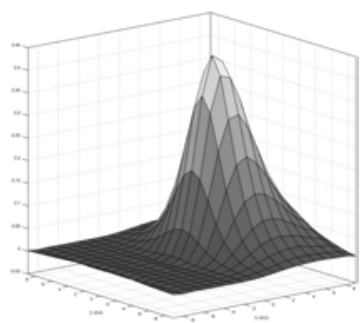

Figure 5.41: An optimized quadrature filter in the frequency domain.

resented by a point or a vector alone. A structure to englobe the total information for each voxel is therefore needed.

The need of storing the contribution from each direction for each voxel has been encountered in many fields, where physical properties need to be locally described. A mathematical field has emerged where tensors can englobe all the information in each voxel. The notion of tensor should not to be confounded with the muscle type, such as the tensor tympani used to dampen sounds when for instance chewing. Derivation of the tensor analysis is briefly stated in the following section.

\subsubsection{Tensor analysis}

While a vector is defined as a list of values and a matrix is defined as a table of values, the natural evolution of such taxonomy would be a list of tables or a table of lists. Therefore, tensors can be seen as a generalization of matrix of $\mathrm{N}$ dimension. When considering more than one direction, such as in the case of the quadrature filtering with the contribution from the six different orientations, a tensor is an adequate solution.

The next step in this study is to compute the tensor, which will include all the local structures derived from the quadrature filtering from the original data from all six directions. The naming of structure tensor will be used 
during the rest of the manuscript. The estimate of the structure tensor can now be constructed as

$$
\mathbf{T}_{e s t}=\sum_{k=1}^{6}\left|q_{k}\right| M_{k}
$$

with $\left|q_{k}\right|$ being the absolute value of the quadrature filter output, and $M_{k}$ is defined as

$$
\alpha \hat{\mathbf{n}}_{k} \hat{\mathbf{n}}_{k}^{T}-\beta \mathbf{I}
$$

where $\hat{\mathbf{n}}_{k}$ is the orientation of the quadrature filter $k, \mathbf{I}$ is the identity matrix, $\alpha$ is $5 / 4$, and $\beta$ is $1 / 4$ in the $3 \mathrm{D}$ case, see[16] for more details on the choice of $\alpha$ and $\beta$. Because the tensor matrix is symmetric, the tensor components can also be written as follows (for the 3D case):

$$
\mathbf{T}=\left(\begin{array}{ccc}
t_{11} & t_{12} & t_{13} \\
t_{12} & t_{22} & t_{23} \\
t_{13} & t_{23} & t_{33}
\end{array}\right)
$$

The advantage of using a tensor resides in the possibility of extracting the eigenvalues and eigenvectors from the local structures. The eigenvalues and eigenvectors inform us about the energy contribution of the signal at the particular voxel in a local neighbourhood in particular orientation. The main reason of extracting the eigenvalues and eigenvectors is the possibility to visualize such information through the use of ellipsoids. Ellipsoids are defined with three axes, the eigenvectors, and the magnitude defined along each axis determines the shape of the ellipsoid through the eigenvalues, see Fig. 5.42.

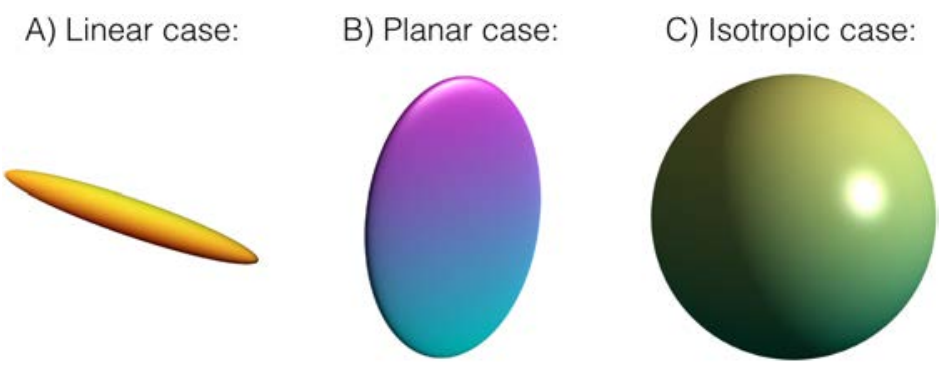

Figure 5.42: Ellipsoids defining: (A) the linear case with $\lambda_{1} \gg \lambda_{2} \simeq \lambda_{3}$, (B) the planar case where $\lambda_{1} \simeq \lambda_{2} \gg \lambda_{3}$, and (C) the isotropic case where $\lambda_{1} \simeq \lambda_{2} \simeq \lambda_{3}$.

Specific combinations of eigenvalues and eigenvectors can then be defined. Linear structures (also known as prolate or cigar-shaped structure), can be extracted when $\lambda_{1} \gg \lambda_{2} \simeq \lambda_{3}$. $\lambda_{1}$ is the dominant componant, while $\lambda_{2}$ 
and $\lambda_{3}$ are similar but much smaller in magnitude compared to $\lambda_{1}$, see Fig. $5.42(\mathrm{~A})$.

Planar structures (also known as oblate or disc-like) can be extracted when $\lambda_{1} \simeq \lambda_{2} \gg \lambda_{3}$. In this case, $\lambda_{1}$ and $\lambda_{2}$ are similar and much larger than $\lambda_{3}$. Therefore the two dominant components $\lambda_{1}$ and $\lambda_{2}$ form a plane with a slight protrusion along $\lambda_{3}$, see Fig. 5.42(B).

Isotropic structures (also known as spherical) can be extracted when $\lambda_{1} \simeq$ $\lambda_{2} \simeq \lambda_{3}$. In that case, because all $\lambda s$ are equivalent, the ellipsoid becomes a sphere without any specific orientation and therefore called isotropic, see Fig. 5.42(C).

To visualize the results from analysis such as the structure tensor analysis along with the original data, volume rendering is very helpful. The theory behind volume rendering is by far beyond the topic of this thesis. Good references exist about this field of research, among others [13]. Some features that were extensively used during this work are explained in the following section.

\subsection{Volume rendering}

Compared to visualization of the data in 2D through slicing back and forth, volume rendering allows rotating the loaded data in all possible directions in an interactive manner. Hiding unwanted structures, without removing the information from the original data is also possible, allowing for instance to only visualize bone. Hiding data can be done in two manners and possibly a combination of the two methods when necessary. The first method is through the use of a transfer function, and the second method relates to the use of clipping planes, detailed in the following.

\section{Transfer function}

A transfer function is a technique to assign a colour (RGB: red green and blue) or a gray shade for each voxel in the $3 \mathrm{D}$ volume. To enable the hiding of voxels that are not of interest, an parameter is used to set the opacity (opposite to transparency) for each voxel, commonly called $\alpha$. Together, the combination of the color and the alpha value is commonly known as a $\operatorname{RGB} \alpha$ value per voxel.

A one-dimensional transfer function maps the $\mathrm{RGB} \alpha$ value for every grayscale intensity from the original data volume. A typical example is [0, 255]. Multi-dimensional transfer functions allow multiple $\operatorname{RGB} \alpha$ values to be mapped to a single isovalue, but this topic is left to the reader to read more about it in [13]. 
For ease of manipulation, the transfer function is sometimes overlaid on a the histogram of the visualized data. Interactive manipulation of the transfer function allows setting some control points on a curve, typically a piecewise linear function where the breaking points are defined by the control points. By setting a specific colour for these control points, it is possible to create linear colour gradients between two colours. The vertical placement of the breakpoint defines the opacity level, with a magnitude of 0 reflecting that specific grayscale value to be set to transparent, while a breakpoint positioning at a value of 1 allows this grayscale value to be fully opaque.

The default transfer function, usually found in volume rendering, is a linear ramp ranging from 0 for the lowest grayscale intensity setting all the black pixels as transparent, and linearly increasing up to 1 for the highest grayscale intensity. For 2D medical images, this type of transfer function is not very useful, but it becomes practical in 3D. Hiding all black voxels from the data in $3 \mathrm{D}$ allows to visualize the objects of interest, while discarding the air and noise around the objects of interest. If not hidden, the visualized data will simply be a black cube.

Different examples of volume rendering are given in Fig. 5.43. For each example, the transfer function is available below the rendering.

In Fig. 5.43(A), the default ramp is used all the way from the low grayscale value to the highest grayscale value. In Fig. 5.43(B), colours are assigned to some of the control points. Blue represents air, brown represents soft tissues with two different types of brown to give a more realistic feeling, while bone is defined as different grayscale values to respect the traditional way of visualizing bone in volume rendering.

One can observe the air within the mastoid air cells while the brown in some of the cells clearly indicates that these cells pertains to trabecular spaces containing bone marrow. In Fig. 5.43(C), the blue colouring is removed and all air voxels are hidden by setting the opacity down to 0 . In Fig. 5.43(D), the transfer function is modified so that only transition between bone and all remaining structures is visible. This allows to provide information about the internal content of the bone.

The right side of the transfer function in Figs. 5.43(A), 5.43(B), and 5.43(C) contains a linearly decreasing ramp down to 0 . These respective ramps are intended to remove possible possible artefacts that contain high density grayscale values, when present. Another possibility to hide, but not physically remove, unwanted information is to use clipping planes. 

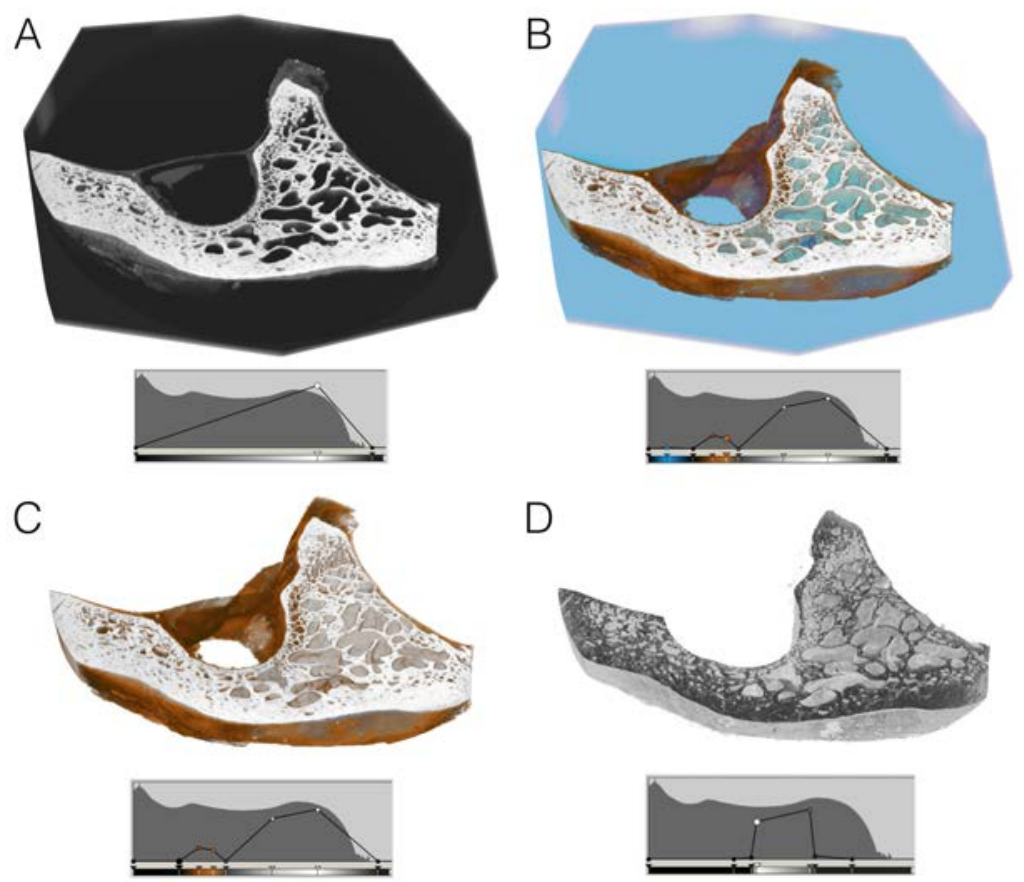

Figure 5.43: Volume rendering of a partial bone scan with varying transfer functions.

\section{Clipping planes}

Clipping planes can be placed around the 3D volume along the six different locations: anterior, posterior, superior, inferior, left, and right. This option allows to interactively hide all voxels in front of these clipping planes, while keeping the remaining voxels behind the clipping planes visible, see Fig. 5.44 .

To resume, in Paper 1, the more basic image processing tools were used to extract the surface area and volume from micro-CT scans of 8 eight temporal bones.

In paper 2, volume rendering with the use of a transfer function such as in Fig. 5.43(D), helped revealing the micro-channels, their orientations, their course, and how they are related to outside structures and to mastoid air cells. Clipping planes were also very useful in hiding unwanted structures to mainly focus on the micro-channels. 

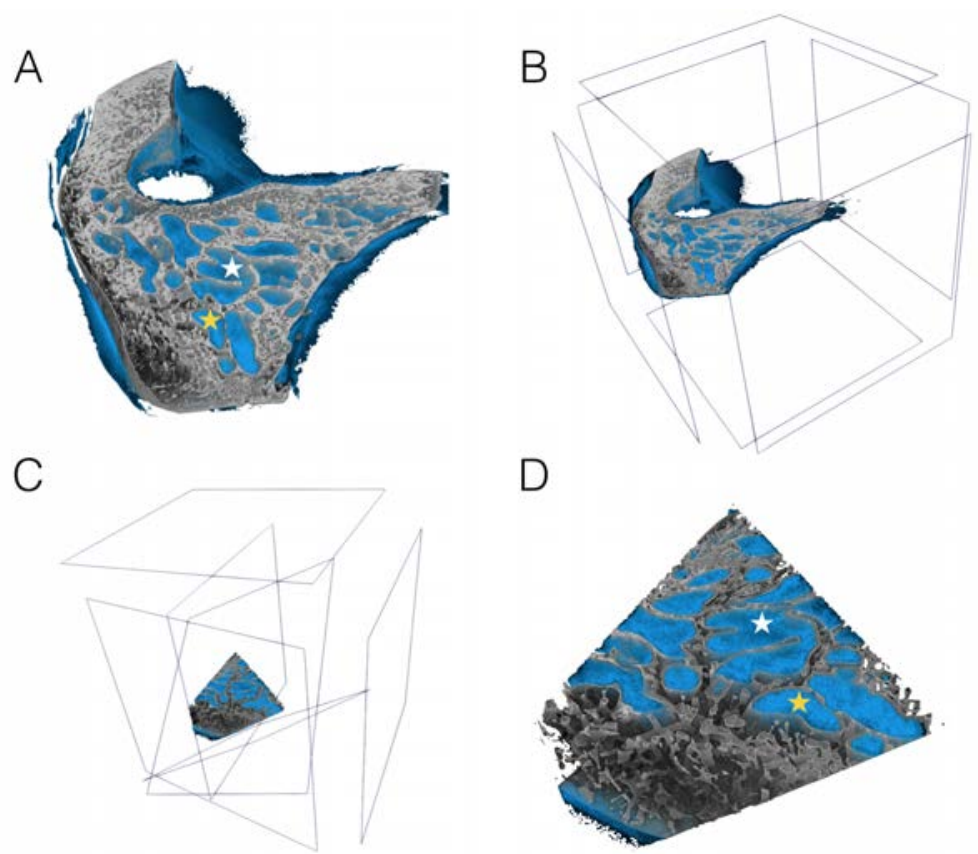

Figure 5.44: Clipping planes used to hide part of the data. A) The original data is fully rendered. B) The initial clipping planes are activated around the full data. C) The clipping planes are interactively displaced and rotated to only expose the part of interest from the original data. D) Only the remaining region of interest is visualized and zoomed to appreciate the details. The white and yellow stars, defining mastoid air cells, are used as references in relation to subfigure A.

In Paper 3, the structure tensor representation can be used when investigating the structural shape of the micro-channels. The ellipsoids are not visualized. Only the eigenvalues of these three specific cases are mapped into one of the R,G,B color channel. From an interpretation point of view, using a structure tensor representation helps understanding the underlying structures from the data; as for instance how tubular the micro-channels are along their path and to reveal elements with a combination of planar and tubular structures inside them as the hub illustrated in Fig. 4(G) or Fig. $4(\mathrm{H})$ in the paper. 


\title{
Contributions of this thesis
}

\author{
"Ex nihilo nihil fit." \\ Nothing comes from nothing.
}

\subsection{Introduction}

Three different but yet related contributions are presented in this thesis. The first contribution concerns the determination of the surface area and volume, in order to estimate the surface area to volume ratio of the mastoid air cell system through the use of micro-CT. The second contribution concerns the discovery of micro-channels within the mastoid bone, hypothetically providing an extra blood supply to the mucosa lining the mastoid air cell system. A preliminary investigation of these micro-channels was then performed. The third contribution involves a more in-depth analysis of these discovered micro-channels, through a structure tensor analysis. The first section details the results obtained from the first contribution. The second section is devoted to the description of the micro-channels while the third section introduces an analysis of their structural shapes.

\subsection{Contribution 1. Surface area and volume of the mastoid air cell system}

Despite the interest demonstrated by recent studies in this field of research, only a few studies have determined the surface area of the mastoid air cell system, [37] [41]. The reason for the lack of studies are multi-fold and are partially introduced in Paper 1.

The number of mastoid air cells observed on a slice from a clinical CT scanning, is far lower than what can be observed on an exposed mastoid from a dry bone. Therefore, clinical CT scanning does not represent the full range of air cell size, and segmenting the mastoid air cells based on clinical 
CT scanning will lead to an inaccurate estimate. The usual segmentation procedure used in previous studies relies on a global threshold technique. If the threshold is set too low, the segmented air cells will appear smaller than their real size, and the opposite for a threshold set too high.

When performing a statistical analysis on the size of the mastoid air cells based on clinical CT scans, all these combined effects will have a much larger effect on the estimation of the surface area than on the volume calculation, especially for the complete MACS.

A possible solution is to increase the resolution of the image data. Small air cells that were hampered by partial volume effects on clinical CT scans are well represented when using micro-CT. Similarly, the septae are now well defined on the micro-CT scans. A longer exposition time combined with a higher X-ray dose can be achieved by using micro-CT scanning, allowing such increase in resolution. To determine the surface area and volume of the mastoid air cell system, four main steps were established in order to extract the mastoid air cell system from the rest of the scanned data.

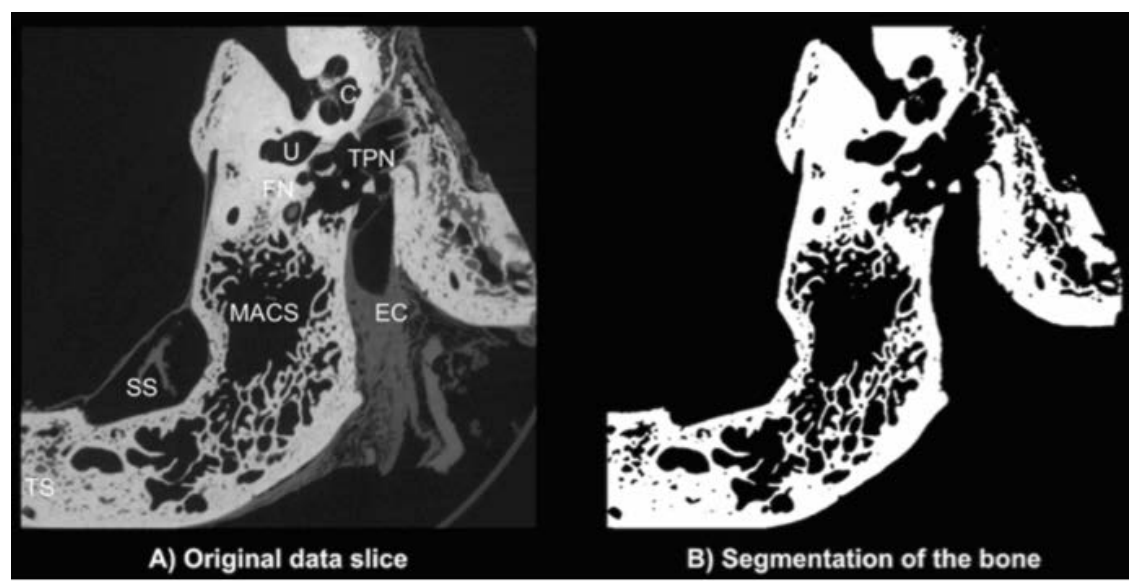

Figure 6.1: A) Axial slice from the original data at the level of the tympanum for bone 5. B) Segmentation of the same bone displaying only the bony parts by using thresholding. Legend. C: cochlea, EC: ear canal, FN: facial nerve, MACS: mastoid air cell system, SS: sigmoid sinus, TPN: tympanum, TS: trabecular spaces.

The first step aimed at separating the temporal bone from all other types of structure in the data, by segmenting the original scan data, visible in Fig. 6.1(A), using simple thresholding technique, see Fig. 6.1(B). Because the bone naturally contains many spaces either filled with soft tissues such as the trabecular spaces, or filled with air such as the mastoid air cells, the 3D segmented binary bone data contained many cavities, see Fig. 6.1(B). 
The second step was then to fill all the holes within the binary bone mask while closing any possible opening to the outside air, see Fig. 6.2(A), and to reassign the original data on the full binary bone mask, see Fig. 6.2(B).

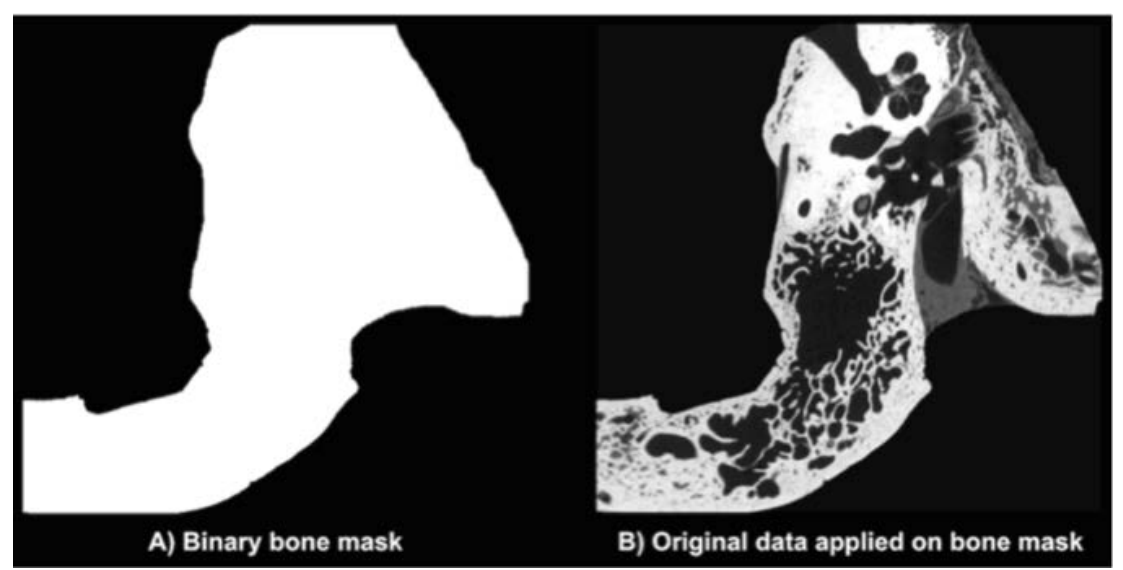

Figure 6.2: A) Filling the bone mask to include all internal structures within the binary mask. B) Re-applying the original data on the filled bone mask.

The third step consisted of two consecutive operations: segmenting out the mastoid air cell using another threshold on the masked thresholded result from the previous step, see Fig. 6.2(B). The threshold was empirically decided based on the air density for each bone specimen, see Fig. 6.3(A).

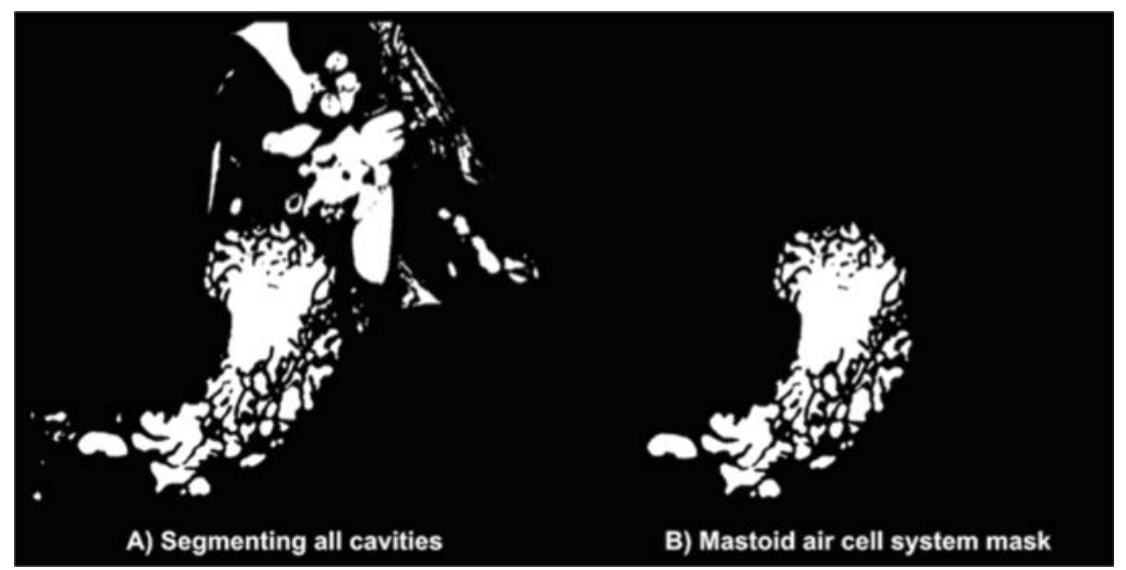

Figure 6.3: A) Extraction of all cavities within the bone using a second thresholding. B) Extraction of the mastoid air cell system from all other cavities.

However, the segmented air included all possible airspaces including the 
mastoid air cell system, along with the tympanum and other unwanted structures. Therefore manual separation of the mastoid air cells from unwanted structures was needed and performed through region growing, see Fig. 6.3(B).

The fourth step ensured the inclusion of all possible voxels containing air, for the final estimate of the surface area and volume of the mastoid air cell system. Voxels at the transition between air cells and the surrounding bone, known as transition voxels, contain a mixture of different structures such as air, bone, and eventually some percentage of mucosa when the size of a voxel is close to the thickness of the mucosa, i.e. around $40 \mu \mathrm{m}$. To cope with partial effect, the binary data representing the mastoid air cells only was dilated, by adding one or more layers of foreground voxels around the segmented mastoid air cells, to include these transition voxels.

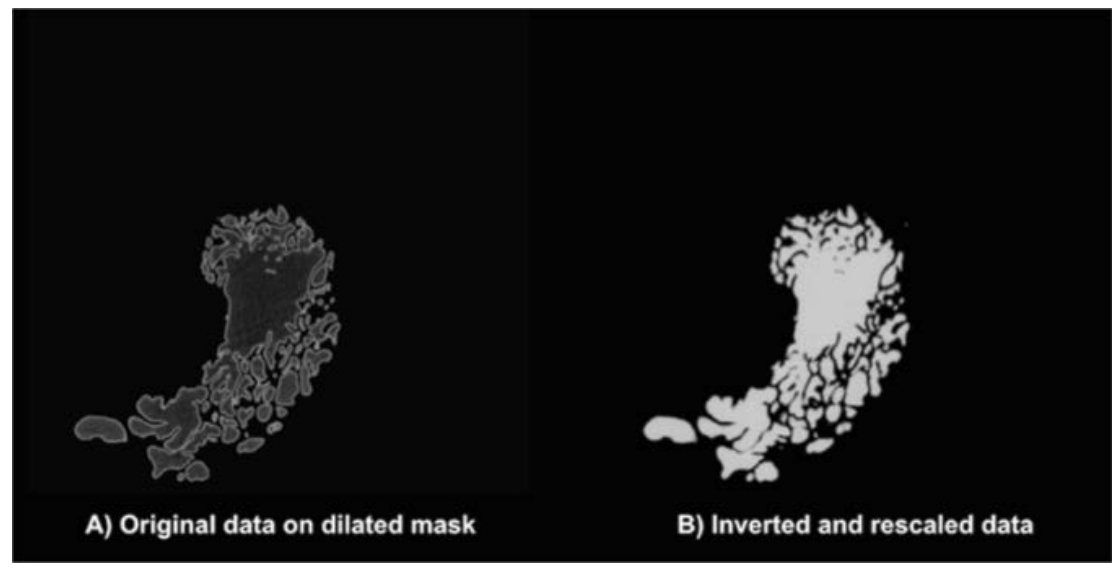

Figure 6.4: A) Original data applied on the dilated version of the mastoid air cell binary mask. The lighter gray border represents the transition voxels between air within the air cells and the bone surrounding them. B) Inversion and rescaling of the original data reapplied on the mastoid air cell system binary mask for the final calculations. The darker gray border respectively represents the transitions voxels in an inverted scale.

The dilated binary representation of the mastoid air cell system was reassigned its original values, giving a masked mastoid air cell system as represented, see Fig. 6.4(A). The white line surrounding the mastoid air cells represents all transition voxels, see Fig. 6.4(A). To give more weight to the air content, seen as dark gray part in Fig. 6.4(A), rather than on the bone content, the grayscale data of the masked mastoid air cell system was inverted while keeping the background unchanged. The grayscale values were then rescaled from 0 to 1 , to express the quantity of air present in 
each voxel in terms of percentage; 1 meaning a voxel fully filled with air while 0 meaning a voxel only containing bone, see Fig. 6.4(B).

Estimation of the final volume for the complete mastoid air cell system was possible by accumulating the air content in each voxel and multiplying the resulting sum by the volume of one voxel. The rescaled grayscale representation of the mastoid air cell system was therefore thresholded at the value of 0.5. A triangular mesh was then created from the $3 \mathrm{D}$ volume and the final surface area was estimated based on the triangular mesh, see Fig. 6.5.

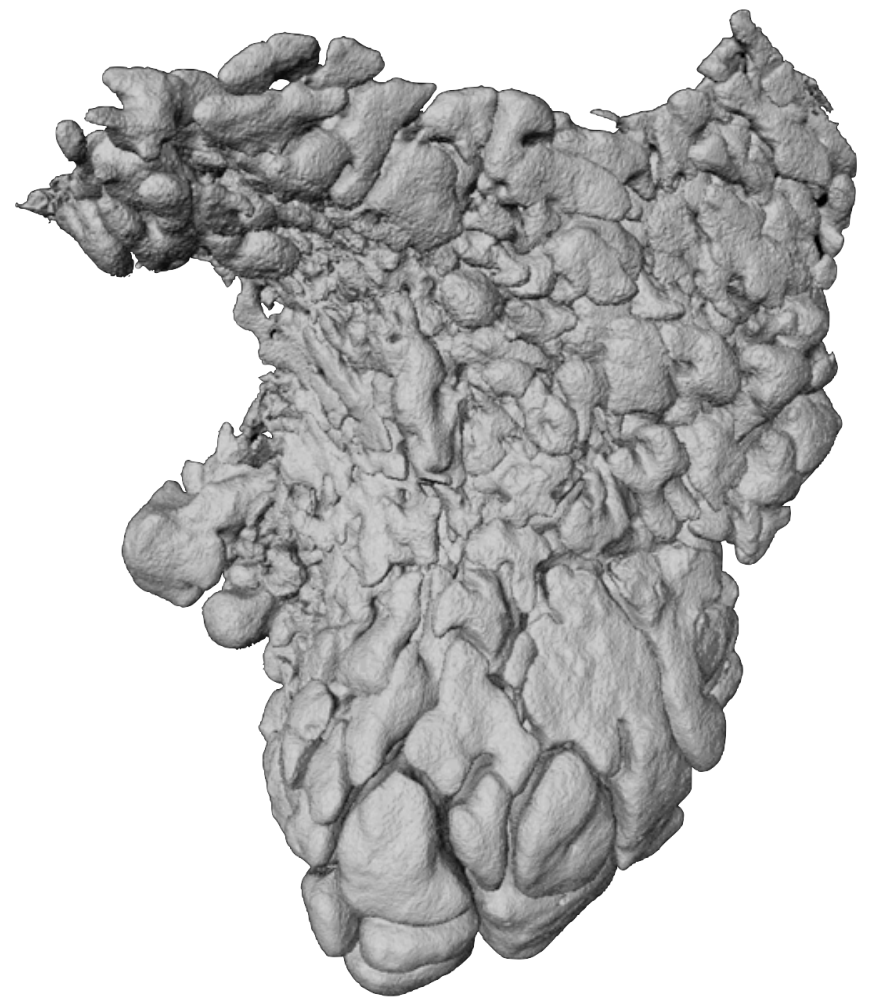

Figure 6.5: A segmented mastoid air cell system from which the surface area can be estimated.

The surface area to volume ratio was then computed by dividing the estimated surface area by the estimated volume.

To compare the results obtained from this study with previous studies performed on clinical CT scans, the final binary volume representing the mastoid air cell system in Fig. 6.4(B) was downsampled successively 4 
times by a factor of 2 for each dimension (X, Y, Z); thus reducing the size of the binary volume by $2^{3}$, also known as an octave, for each iteration.

This downsampling operation was performed four times leading to five volumes with resolutions ranging from the original resolution to a resolution close to a conventional clinical CT scanner, i.e. approximately $600 \mu \mathrm{m}$.

For each downsampling level, the volume and the surface area were estimated accordingly and the surface to volume ratio derived. The results are taken from Paper 2. However, for clarity, these results are repeated in the following of the section.

Fig. 6.6 resumes the measured surface area $\left(\mathrm{cm}^{2}\right)$ for the 8 bones plotted against 5 different resolutions represented on a logarithmic scale from their respective original resolution down to a resolution found in clinical CT scanners with three intermediate resolutions chosen to allow a logarithmic scale.

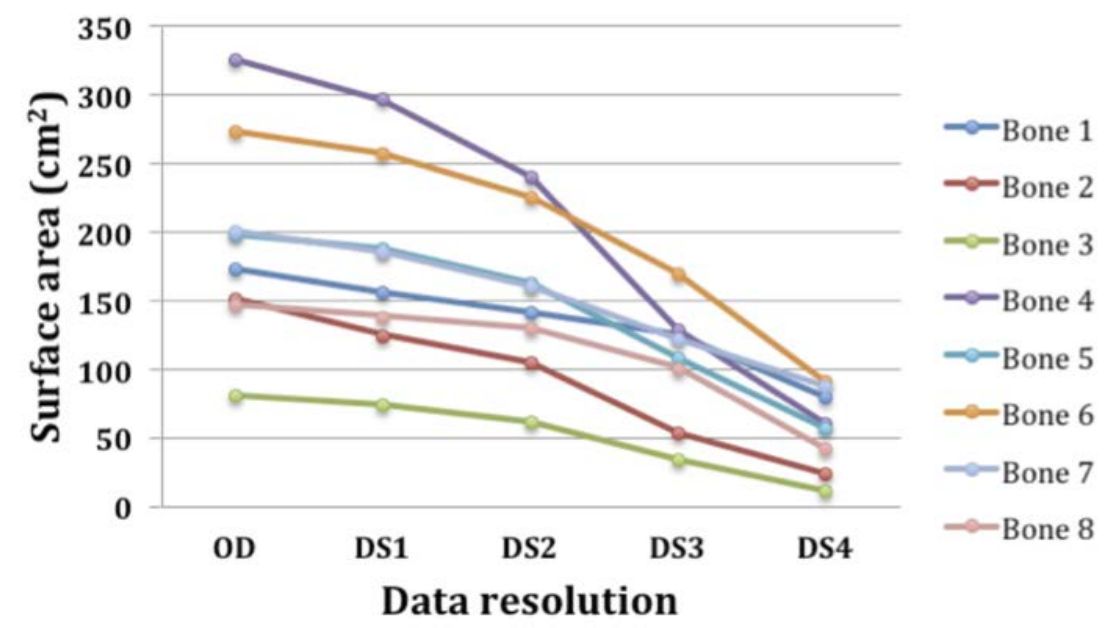

Figure 6.6: Estimated surface area over the different data resolutions (OS: original size, DS1: downsampling 1, DS2: downsampling 2, DS3: downsampling 3, DS4: downsampling 4) for the 8 bone specimens.

The results clearly show a non-linear trend for all bone specimens. For the original resolution (OD), the measured surface areas for all bones picture a large variation, ranging from $74 \mathrm{~cm}^{2}$ (Bone 3) to $297 \mathrm{~cm}^{2}$ (Bone 4) with a mean of $194 \mathrm{~cm}^{2}\left(\mathrm{SD}=76.5 \mathrm{~cm}^{2}\right)$. On the lowest resolution (DS4), the measured surface areas for all bones form a much narrower range of values between $24 \mathrm{~cm}^{2}$ (Bone 3) to $91 \mathrm{~cm}^{2}$ (Bone 6) with a mean of $56.5 \mathrm{~cm}^{2}$ $\left(\mathrm{SD}=29.6 \mathrm{~cm}^{2}\right)$. 


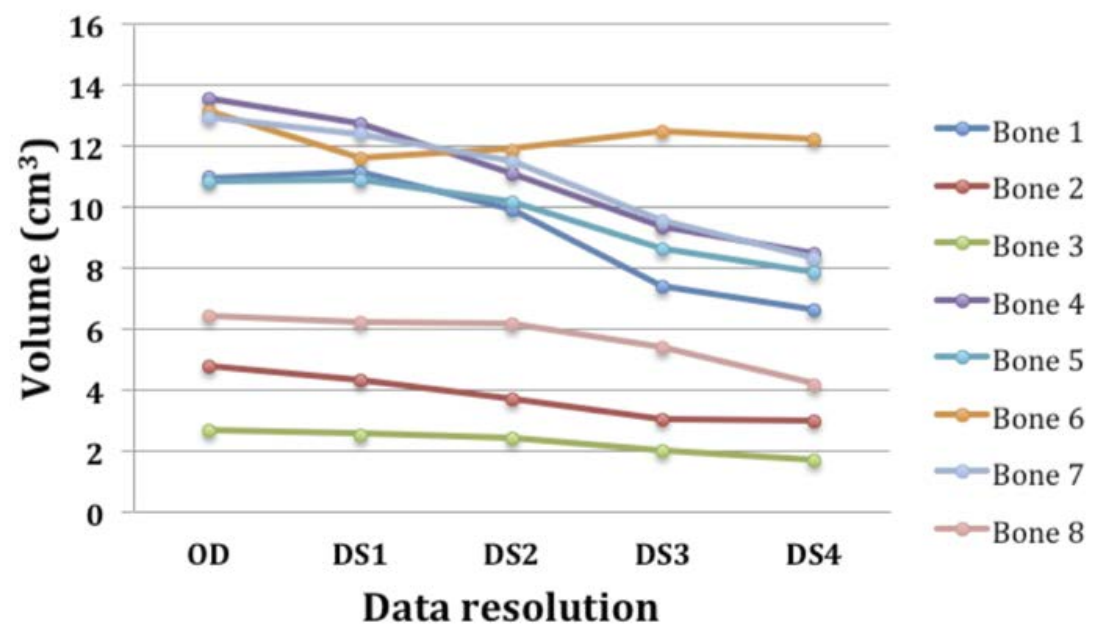

Figure 6.7: Plot of the volume over the different data resolutions (OS: original size, DS1: downsampling 1, DS2: downsampling 2, DS3: downsampling 3, DS4: downsampling 4) for the 8 bone specimens.

In Fig. 6.7, the corresponding volume measurements $\left(\mathrm{cm}^{3}\right)$ for the 8 bones are plotted with the same logarithmic scale as in Fig. 6.7. Whereas the volume measurement for bones $2,3,6$, and 8 can be seen as varying linearly over the different scan resolutions, the volume measurement for bones 1 , 4, 5, and 7 present a more non-linear pattern, though following a similar trend when compared with each others. For the highest resolution (OD), the mean value is $9.5 \mathrm{~cm}^{3}\left(\mathrm{SD}=4.2 \mathrm{~cm}^{3}\right)$ while for the lowest resolution $(\mathrm{DS} 4)$, the mean value is $6.5 \mathrm{~cm}^{3}\left(\mathrm{SD}=3.5 \mathrm{~cm}^{3}\right)$.

Fig. 6.8 illustrates the surface area to volume ratios $\left(\mathrm{cm}^{-1}\right)$ also plotted with the same logarithmic scale as in Fig. 6.6 and Fig. 6.7 for the 8 bones. Because the volume measurements are rather constant compared to the measured surface areas, the estimated surface area to volume ratios follow the same patterns as for the measured surface areas, see Fig. 6.8. The mean value for the highest resolution is $22.37 \mathrm{~cm}^{-1}\left(\mathrm{SD}=6.12 \mathrm{~cm}^{-1}\right)$. The mean value for the lowest resolution $(\mathrm{DS} 4)$ is $8.5 \mathrm{~cm}^{-1}\left(\mathrm{SD}=2.02 \mathrm{~cm}^{-1}\right)$. The variation of the surface area to volume ratio between the lowest resolution and the highest resolution can be resumed by an amplification factor of 3 .

The results from this study, resumed in Table 6.1, show that the mean surface area estimated from micro-CT scans varies by a factor of about 3.5 when compared to the mean surface area estimated from the simulated clinical CT, with a resolution of approximately $625 \mu \mathrm{m}$ (Table 2). For the volume, the mean volume from the micro-CT scan resolution compared 


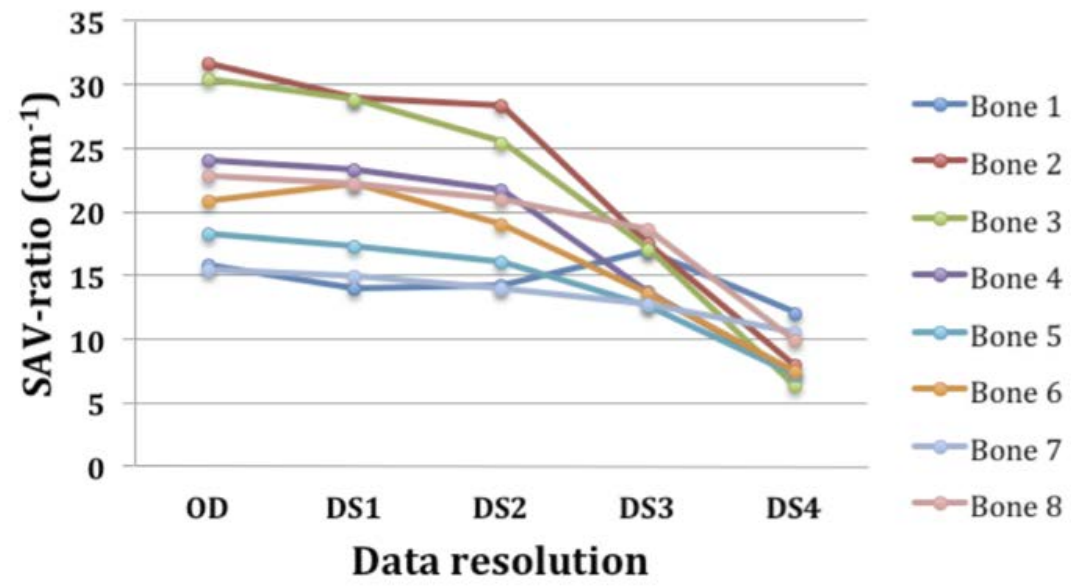

Figure 6.8: Plot of the surface area to volume ratio over the different data resolutions (OS: original size, DS1: downsampling 1, DS2: downsampling 2, DS3: downsampling 3, DS4: downsampling 4) for the 8 bone specimens.

to the simulated clinical CT scan resolution is about 1.5 times higher. The ratio between the mean surface area to volume ratio from the highest resolution against the mean surface area to volume ratio from the lowest resolution gives a factor of 2.63, indicating that the undetected air cells clearly have an effect on the measurement of the surface area rather than on the measurement of the volume.

Observation of the results given for DS1, DS2, and DS3 in Table 2 while observing the trends for the curve for each bone on Fig. 6.6 and Fig. 6.8, clearly show the resolution level beyond which an abrupt change in the results can be observed, namely DS2. For the mean surface area measurement, the transition between the original resolution and the first downsampling, DS1, corresponds to a degradation of $20 \mathrm{~cm}^{2}$. The same applies between DS1 and DS2. Beyond DS2, the mean surface area drops by approximately $50 \mathrm{~cm}^{2}$ between DS2 and DS3 and equivalently between DS3 and the lowest resolution DS4. Because the volume measurements are fairly constant over the different resolutions, the surface-area to volume ratios follow a similar trend.

The volume measurements found in this study seems in line with all previous studies, which further strengthen the fact that volume measurement is independent of the scan resolution.

The uniqueness of such a study lies in the first attempt to estimate the surface area to volume ratio from micro-CT scanning of the mastoid air 


\begin{tabular}{lcccccc}
\hline Statistics & & Original data & DS1 & DS2 & DS3 & DS4 \\
\hline \hline SA & Mean & 193.70 & 177.49 & 153.27 & 105.12 & 56.41 \\
$\left(\mathrm{~cm}^{2}\right)$ & SD & 76.56 & 72.05 & 59.08 & 43.42 & 29.60 \\
& Min & 81.05 & 73.68 & 61.42 & 34.26 & 10.66 \\
& Max & 325.95 & 296.64 & 240.25 & 169.95 & 91.19 \\
& Range & 244.90 & 222.96 & 177.83 & 135.69 & 80.53 \\
\hline VOL & Mean & 9.43 & 9.00 & 8.37 & 7.24 & 6.56 \\
$\left(\mathrm{~cm}^{3}\right)$ & SD & 4.21 & 4.00 & 3.73 & 3.54 & 3.44 \\
& Min & 2.67 & 2.56 & 2.42 & 2.01 & 1.70 \\
& Max & 13.58 & 12.74 & 11.90 & 12.49 & 12.25 \\
& Range & 28.91 & 10.18 & 9.48 & 10.48 & 10.55 \\
\hline $\mathrm{SA} / \mathrm{V}$ & Mean & 22.37 & 21.42 & 19.93 & 15.32 & 8.55 \\
$\left(\mathrm{~cm}^{-1}\right)$ & SD & 6.12 & 5.72 & 5.20 & 2.41 & 2.02 \\
& Min & 15.43 & 13.97 & 13.91 & 12.55 & 6.27 \\
& Max & 31.58 & 28.84 & 28.31 & 18.60 & 12.03 \\
& Range & 16.15 & 14.87 & 14.40 & 6.05 & 5.76 \\
\hline
\end{tabular}

Table 6.1: Statistics resuming all computations.

cell system of human temporal bone.

The time used for the overall study was long, but is believed to be beneficial in providing additional knowledge about the mastoid air cell system in terms of statistical descriptors. To keep the reproducibility of the study and gain the trust from the reader, the image processing tools used in this study were kept as simple as possible. This decision was also made so that results from much more advanced image processing, being developed and customized, can be compared to the methods used in this study.

This study has been reported in a manuscript being submitted for revision for the coming special issue of the Hearing Research for the Middle Ear Mechanics in Research and Otology international symposium for 2015 to be held in Aalborg in Denmark.

The following contribution relates to a discovery of micro-channels present in the mastoid bone both at the outside of the mastoid air cells as well as between the air cells.

\subsection{Contribution 2. Discovery of micro-channels within the mastoid bone}

By visual inspection of micro-CT scanning data, intended for the measurement of the surface area and volume of the mastoid air cell system as presented in the first contribution, numerous micro-channels were found. 
These micro-channels have not been described in previous literature. These micro-channels are likely to represent a separate blood supply for the mastoid mucosa, and have important implications for further understanding the middle ear physiology including its pressure regulation and gas exchange.

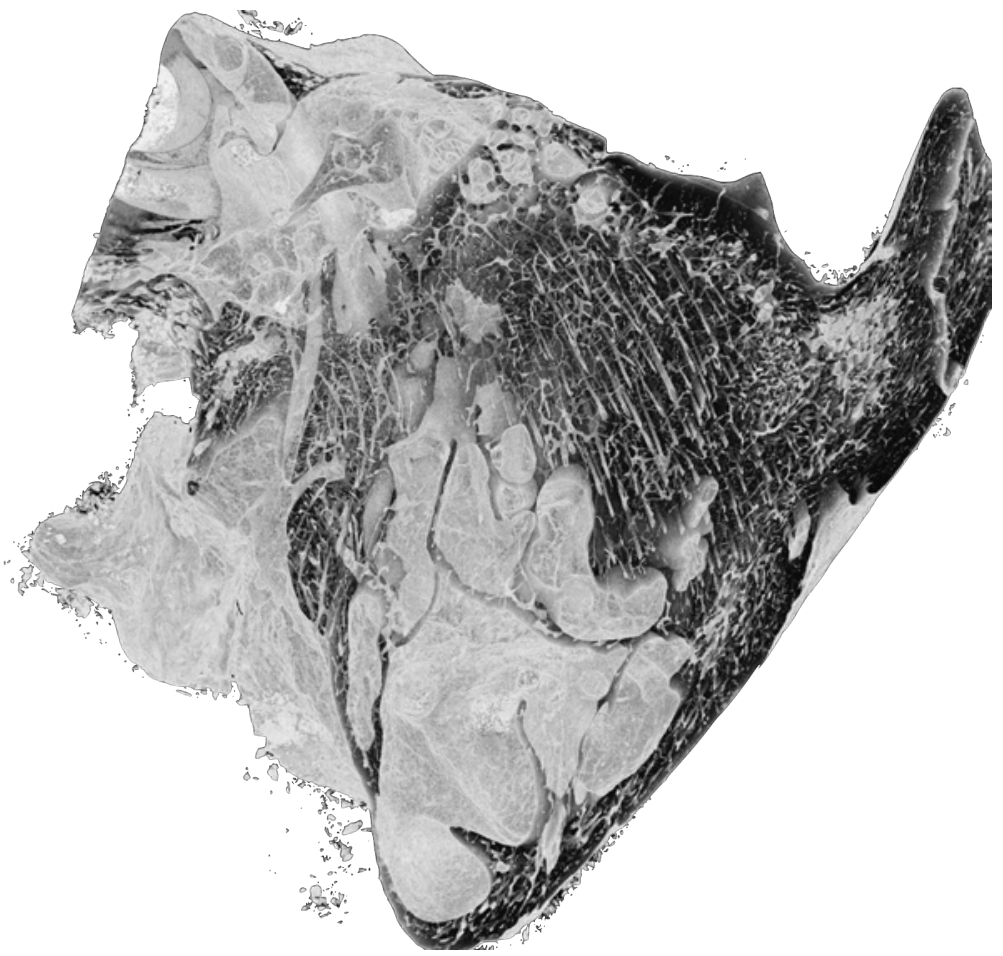

Figure 6.9: Volume rendering of a bone specimen.

Discovery of these micro-channels originated from 3D visualization of the scanned bone through volume rendering, with a special transfer function, manually adjusted to only visualize the transition between bone and all other types of structures, see Fig. 6.9.

Volume rendering of the micro-CT data revealed two main types of microchannels:

- Parallel channels which emerged from indentations on the lateral surface of the temporal bone and inside the ear canal, and which traversed the compact bone; many connected directly to the air cells;

- Irregular shaped channels that seemed to follow the shapes of the bony septae between the air cells inside the mastoid, as well as they formed network by branching and connections between adjacent air cells. 
In several locations, more than one channel were found to be connected to individual air cells, see Fig. 6.10 (white arrows). Direct communication between the mastoid with the external part of the bone through microchannels was also observed, see Fig. 6.10 (black arrows).

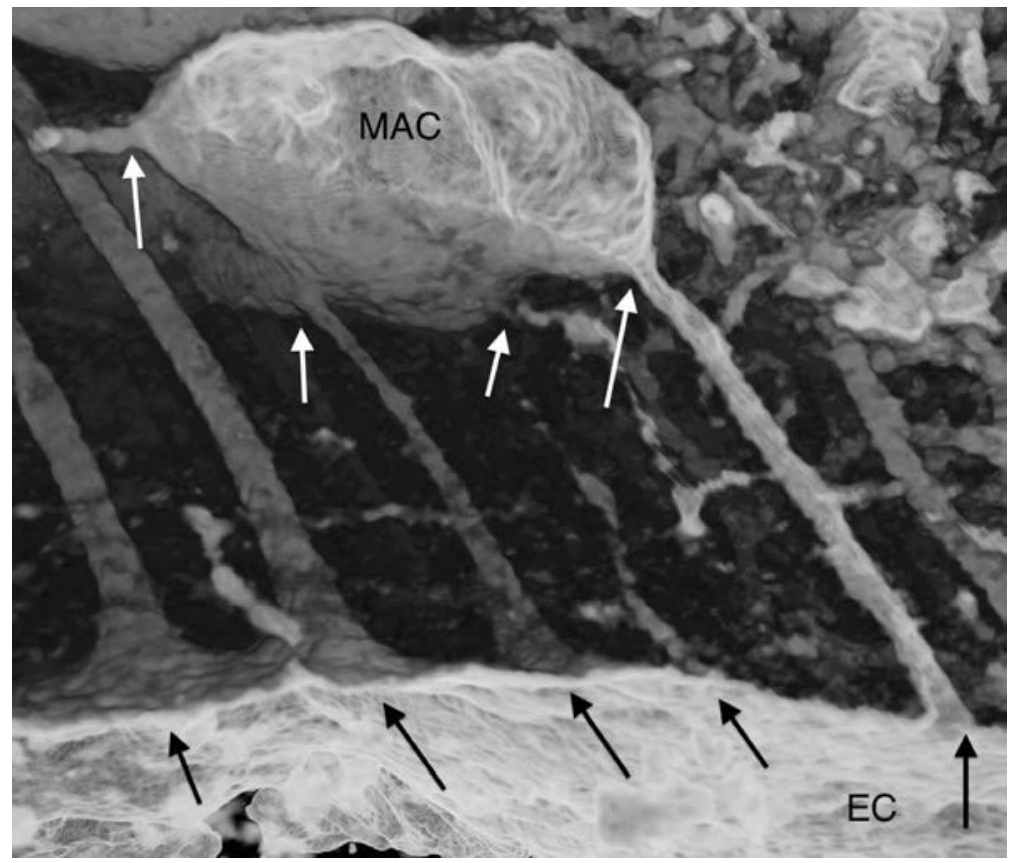

Figure 6.10: Multiple micro-channels communicating with a single mastoid air cell (white arrows). Some of micro-channels are also in direct communication with the exposed part of the wall of the ear canal through small indentations (black arrows).

The structural properties of the micro-channels resemble a vascular network. Considering their mean diameter of $158 \mu \mathrm{m}$, the micro-channels could contain both small arterioles and venules, which have diameters around $30 \mu m$ and $10-60 \mu m$ respectively, [7]. Haversian canals are well known in bone for providing a vascular supply for the tissue, but these canals are smaller than $45 \mu \mathrm{m}$ in diameter and usually only contain capillaries, [8]. Such canals were below the resolution of our method $(40-60 \mu \mathrm{m}$ in terms of isotropic voxel size) and were considered as separate from the present discovered micro-channels.

However, branches formed by the micro-channels within the compact part and trabecular part of the bone can be observed in Fig. 6.11, with the white stars along the path of the main micro-channel. This could provide a vascular supply for the bone marrow itself. 


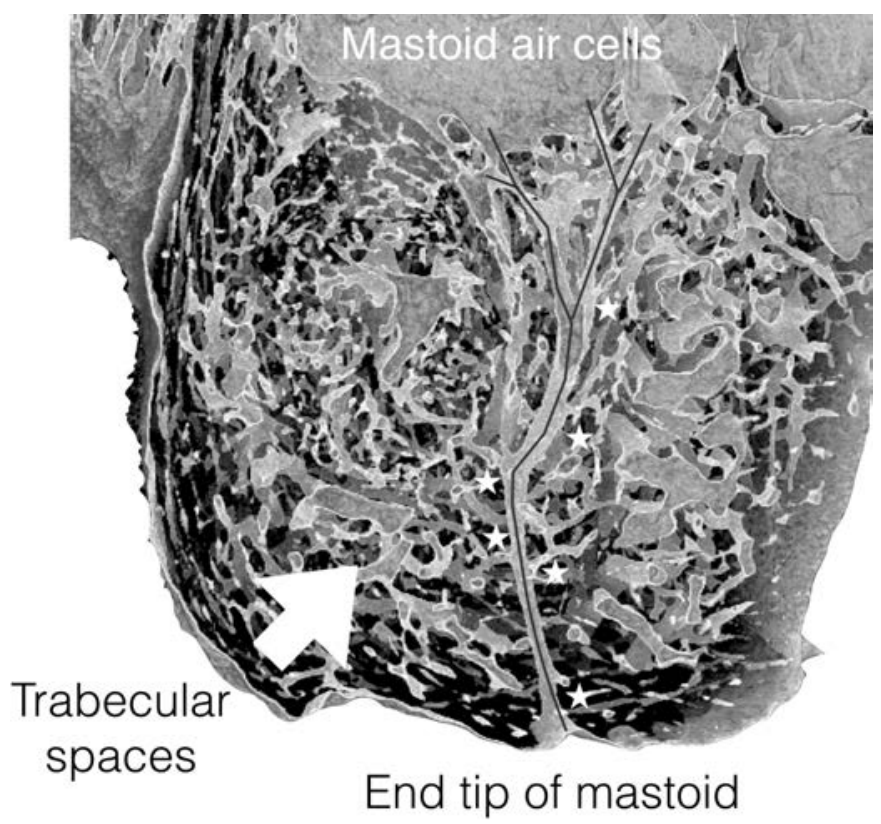

Figure 6.11: Micro-channel at the mastoid tip running through trabecular spaces to reach an apical mastoid air cell (path overlaid on the channel). Notice some small branching (white stars) from the microchannel towards the trabecular spaces, possibly providing blood back and forth to the bone marrow.

Some of the micro-channels also appear to have an end-point at the surface of the bone, see Fig. 6.12. Moreover, since all air cells were connected to several channels, it seemed reasonable to suggest that such a blood supply would be aimed at the mastoid mucosa lining the mastoid air cells.

The main interesting feature of Fig. 6.11, is the fact that the trabecular spaces contain bone marrow housing sinusoidal type of capillaries; hence another plausible hint for these micro-channels to house blood vessels.

As a side note, the trabecular spaces present at the tip of the mastoid are due to the attachment of the splenius capitis, longissimus capitis, digastric posterior belly, and sternocleidomastoid muscles as in Chapt. 3.

In order to investigate the exact content of the micro-channels further histological studies are needed. Fig. 6.13 shows a preliminary histological section from the surface of the mastoid at the posterior part of the ear canal.

This section clearly shows at the center a channel with a diameter of $133 \mu \mathrm{m}$ containing a venole and arteriole, respectively; in addition some connective 


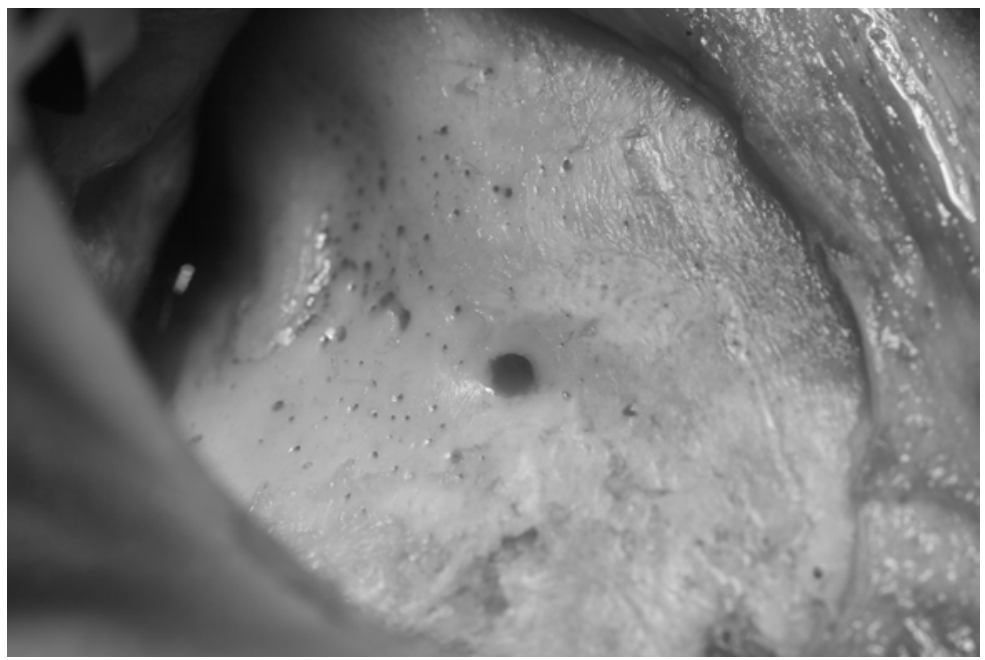

Figure 6.12: Photo of a micro-channel at the level of an exposed mastoid bone during surgery seen as a small indentation at the surface of the bone.

tissue is found and the channel is surrounded by typical bone tissue. Thus, this preliminary investigation supported the idea that the micro-channels may contain vascular elements.

Further investigations are obviously needed for a detailed and systematic description of the mastoid structure, in order to identify the true content of its micro-channels. The difficulty in revealing micro-channels using histological sectioning is the variation in orientation when the micro-channels run over distance. Depending on how the bone specimen was sectioned, it may be impossible to locally reveal any micro-channel and allow a deterministic density measure per square unit.

From a basic clinical research point of view, the current findings may be important, because a supplementary while extensive vascularization permits to get both a high perfusion and effective changes in the vascular congestion. The role of the mucosal perfusion related to gas exchange and pressure regulation is still under debate [31].

During the development of the mastoid, the air cells are forming in a bone matrix that is in constant evolution. Hence, the presence of micro-channels to provide the mucosa lining the mastoid air cells with blood seems to form a separate vascular supply for the mastoid expanding accordingly, but the main role of these micro-channels remains unknown.

The finding of these micro-channels is presented in Paper 2. It was pub- 


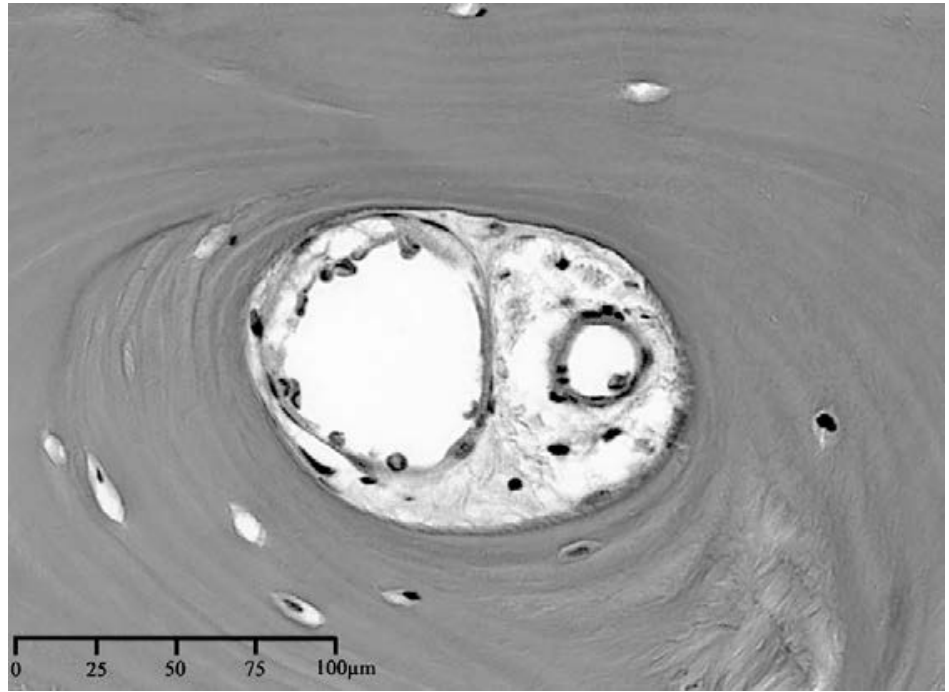

Figure 6.13: Photo of a histological section of a temporal bone at the level of a mastoid where a micro-channel was exposed normal to the section (see colour photo in Paper 2).

lished in the special issue of the Hearing Research for the Middle Ear Mechanics in Research and Otology international symposium for 2012 (Volume 301, July 2013).

\subsection{Contribution 3. Structual analysis of the micro- channels}

As stated in the previous section, these micro-channels resemble a vascular network, which points to a new and separate blood supply for the mucosa of the mastoid air cell system. The micro-channels resemble tubular-like structures and appear to have a wide range of shapes and diameters as illustrated in Paper 2. The resemblance with a vascular network could lead to the use of existing vessel segmentation methods, but through observations based on histological sections, the presence of both arterioles and venules inside were revealed, leading to a need of taking their structural content into consideration.

Local structure tensor analysis based on a second order tensor seems to provide a robust representation of the channels [10]. Local structure tensor analysis have been used in different field of research related to medical image processing, notably in image enhancement via adaptive filtering, [14].

In this thesis, a structural pre-analysis was performed on the micro-channels 
by using local structure tensor analysis based on micro-CT scanning.

The main theory used to extract the structure tensor is detailed in Chapt. 5 and will not be covered in this chapter; but to resume, such structures can be represented using a structure tensor analysis through its corresponding eigenvalues and eigenvectors.

The eigenvalues from the structure tensor can further be used to estimate the probability of each visited neighborhood belonging to either a rank 1 , rank 2 , or a rank 3 tensor, defined by $p_{1}, p_{2}$, and $p_{3}$, where $\sum_{k=1}^{3} p_{k}=1$ which can be seen as probabilities as [16].

$$
p_{1}=\frac{\lambda_{1}-\lambda_{2}}{\lambda_{1}}, \quad p_{2}=\frac{\lambda_{2}-\lambda_{3}}{\lambda_{1}}, \quad p_{3}=\frac{\lambda_{3}}{\lambda_{1}}
$$

Natural structures like the micro-channels are however composed of a mixture of these three cases and a more general ideal structure tensor should instead be represented as

$$
\mathbf{T}=p_{1} \mathbf{T}_{1}+p_{2} \mathbf{T}_{2}+p_{3} \mathbf{T}_{3}
$$

The resulting $p_{1}, p_{2}$, and $p_{3}$ from the estimated structure tensor were assessed using volume rendering through MeVisLab. $p_{1}, p_{2}$, and $p_{3}$ are probability measures of each special case.

To test validity of the results, three different types of objects were analyzed. At first, a set of ellipsoids having different sizes and orientations were processed, see Fig. 6.14.

Fig. 6.14(A) demonstrates the original data, while Fig. 6.14(B,C,D) respectively display $p_{1}$ alone, $p_{2}$ alone, and $p_{3}$ alone.

Fig. 6.14(E) illustrates a mixture of $p_{1}$ and $p_{2}$, while Fig. $6.14(\mathrm{~F})$ shows the mixture of $p_{2}$ and $p_{3}$, and Fig. $6.14(\mathrm{G}, \mathrm{H})$ gives a comparison of the mixture of all cases $p_{1}, p_{2}$, and $p_{3}(\mathrm{H})$ in relation to the original data $(\mathrm{G})$.

Observe that in this last case, the isotropic part is only visible at the extremities of the lower left ellipsoid, enhanced with a zoomed version in the yellow box, and pointed by the thin yellow arrow to the right.

A second case with ellipsoidal cylinders was also tested, see Fig. 6.15.

Fig. 6.15(A) demonstrates the original data, while Fig. 6.15(B,C) respectively display $p_{1}$ alone, $p_{2}$ alone. Fig. 6.15(D) illustrates a mixture of $p_{1}$ and $p_{2}$, while Fig. 6.15(E) enlights the mixture of $p_{2}$ and $p_{3}$, and Fig. $6.15(\mathrm{~F})$ reflects the mixture of $p_{1}$ and $p_{3}$. Fig. $6.14(\mathrm{G}, \mathrm{H})$ compares of the mixture of all cases $p_{1}, p_{2}$, and $p_{3}(\mathrm{H})$ in relation to the original data $(\mathrm{G})$. 
A

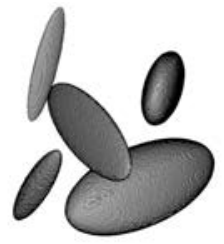

D

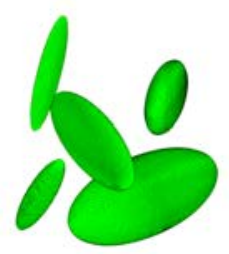

B

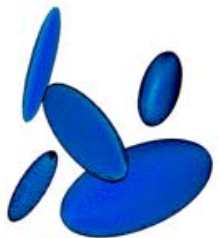

E

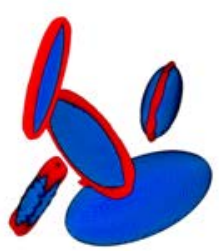

C

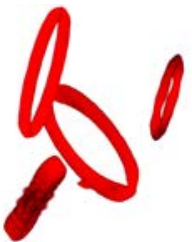

$\mathrm{F}$

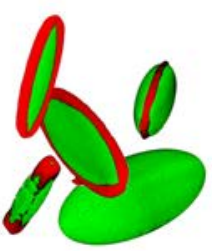

G

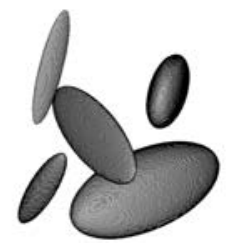

$\mathrm{H}$
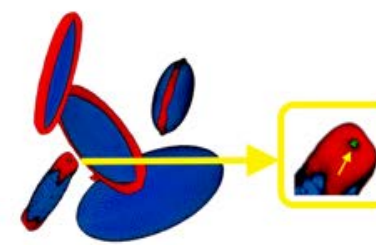

Figure 6.14: Structure tensor analysis on ellipsoids having different sizes and orientations. Legend: A. Original data, B. $p_{1}$ alone (blue), $C$. $p_{2}$ alone (red), D. $p_{3}$ alone (green), E. Mixture of $p_{1}$ (blue) and $p_{2}$ (red), F. Mixture of $p_{2}$ (red) and $p_{3}$ (green), G. Original data, H. Mixture of all cases $p_{1}$ (blue), $p_{2}$ (red), and $p_{3}$ (green). The isotropic is only visible at the extremities of the lower left ellipsoid, enhanced with a zoomed version in the yellow box and pointed by the thin yellow arrow to the right.

As for Fig. 6.14, the isotropic part of Fig. $6.14(\mathrm{H})$ is only visible at the extremities of the ellipsoidal cylinders, enhanced with a zoomed version in the yellow box and pointed by the thin yellow arrow to the right.

At last, a set of cylinders having different orientations but all crossing at their centre were analyzed, see Fig. 6.16.

Fig. 6.16(A2, A3) are two different views of the original data shown in Fig. 6.16(A1) with a slicing through the data to expose the inside of the cylinders and the location where the cylinders cross each others.

Fig. $6.16(\mathrm{~B} 1, \mathrm{~B} 2, \mathrm{~B} 3)$ are the results from the structure tensor analysis where $p_{1}$ (planar) can be observed as blue, $p_{2}$ (linear) as red, and $p_{3}$ (isotropic) as green.

For the isotropic part at the centre of the cylinders, especially visible at the section level in Fig. 6.16(B3), it can also be observed at the extremities of 

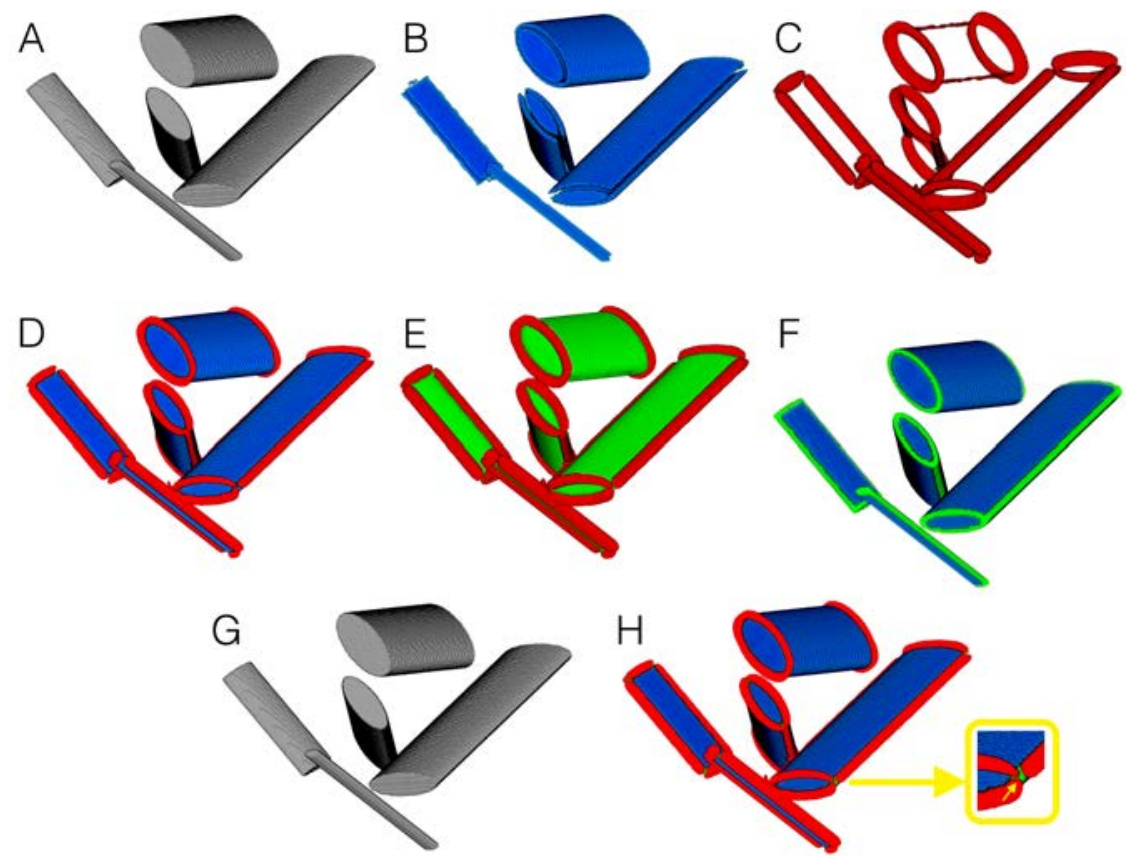

Figure 6.15: Structure tensor analysis on ellipsoidal cylinders having different sizes and orientations. Legend: A. Original data, B. $p_{1}$ alone (blue), C. $p_{2}$ (red), D. Mixture of $p_{1}$ (blue) and $p_{2}$ (red), E. Mixture of $p_{2}$ (red) and $p_{3}$ (green), G. Mixture of $p_{1}$ (blue) and $p_{3}$ (green). $H$. Original data for comparison purposes with $H$, H. Mixture of all cases $p_{1}$ (blue), $p_{2}$ (red), and $p_{3}$ (green). Observe that in this last case, the isotropic is only visible at the extremities of the lower left ellipsoid, enhanced with a zoomed version in the yellow box and pointed by the thin yellow arrow to the right.

each cylinders between the linear and planar structures.

Also the red structures found on the outside of the crossing of all cylinders is due to the sharp transitions as visible in Fig. 6.16(A1), forming a sharp edge interpreted as a linear structure and hence its red color.

A more natural span going from a rank 1 tensor to a rank 2 tensor, with the rank 3 tensor in between, so as to move from a tubular structure towards a planar structure via an isotropic structure is needed. To visualize this natural transition, a lookup table (LUT) was created, where the rank 2 tensor was assigned the red color, the rank 3 tensor was assigned the green color, and the rank 1 tensor assigned the blue color. Each RGB channel was controlled by a respective non-linear function based on sigmoid functions. 

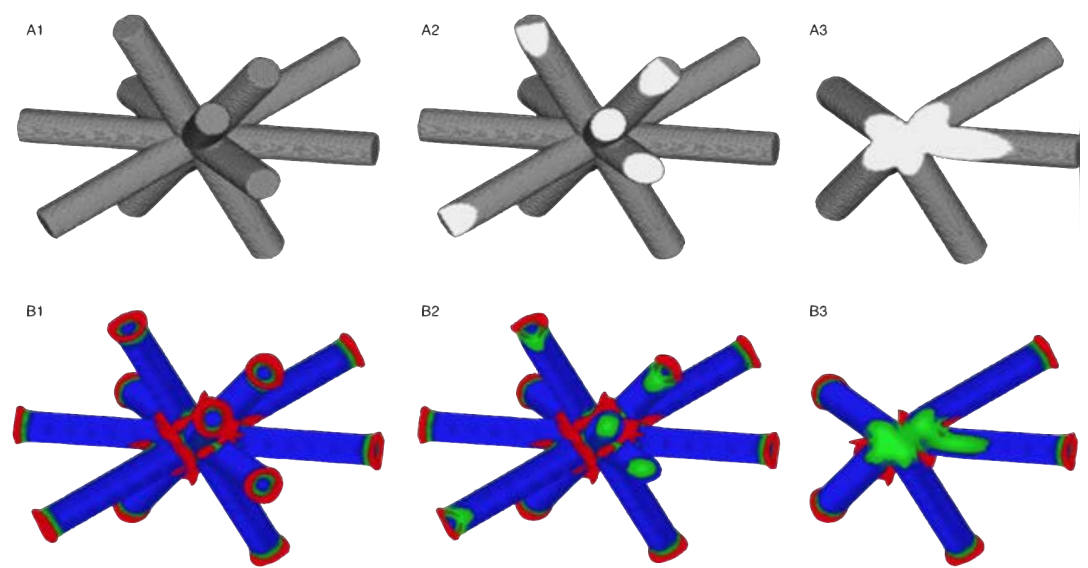

Figure 6.16: Cylinders with different orientations crossing each others at their centre were first analyzed. The linear structures are represented as red, the planar structures depicted as blue, while the isotropic rendered as green

The 3D volume representing all the special cases was created as

$$
p_{m}=p_{1}+\gamma p_{3}
$$

with $\gamma=0.4$, leading to $p_{m}=1$ for rank 1 tensor, $p_{m}=0$ for rank 2 tensor, and $p_{m}=0.4$ for rank 3 tensor.

Figure 6.17 a illustrates all structures at once from the volume $p_{m}$ for the micro-channels.

Figure $6.17 \mathrm{~b}-\mathrm{c}$ respectively represents $p_{1}, p_{2}$, and $p_{3}$ with their corresponding RGB color channel and helps to better understand their complimentary contribution.

Overlaying $p_{1}, p_{2}$, and $p_{3}$ on the original data further informs us about their locations with respect to the bone structures, i.e. within the microchannels, the ear canal (EC), or within the mastoid air cells, see Fig. 6.18 to the right.

To avoid visualizing the structure tensor resulting from the bone itself, the original data was segmented out so that only the transition between bone and other structures was visible.

The resulting binary segmentation was then reassigned the structure tensor result. For better localization of the resulting structure tensor with a indepth perception of the structures in relation to the original data, the results were overlaid on the original data, see Fig. 6.18. 


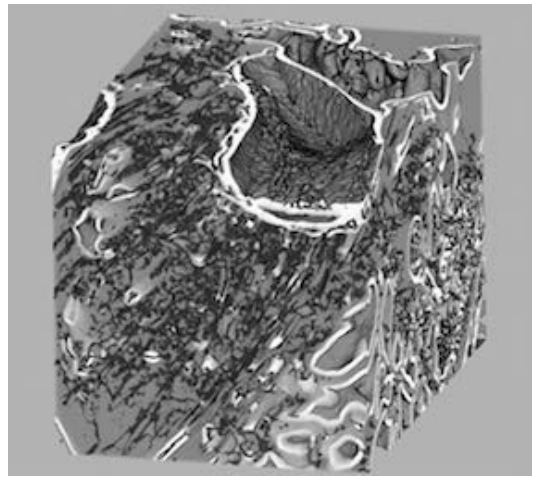

(a) $p_{m}$

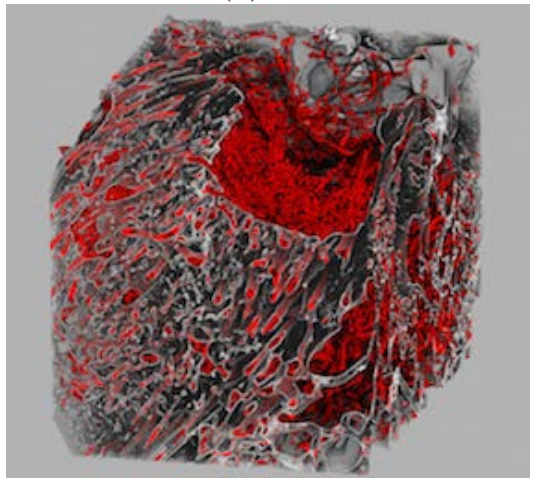

(c) Rank 2 tensor

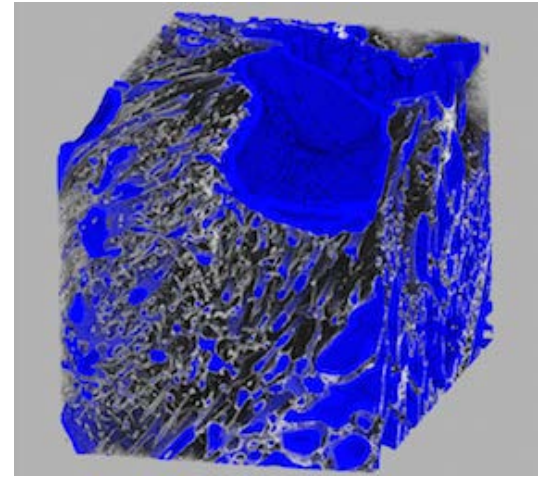

(b) Rank 1 tensor

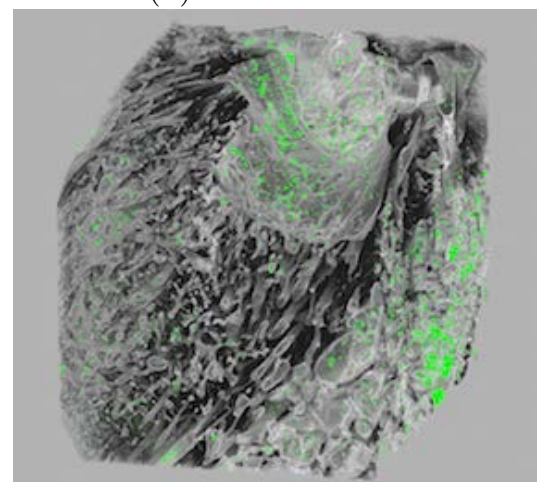

(d) Rank 3 tensor

Figure 6.17: (a): Mixture of all three cases into the single volume $p_{m}$. (b): $p_{1}$ representing planar structures as blue. (c): $p_{2}$ representing line and tubular structures as red. (d): $p_{3}$ representing isotropic structures as green.

A larger scale visualization of the resulting structure tensor analysis is illustrated in Fig. 6.19. Notice the hair follicle, located on the right side of the ear canal (top right of the image) and represented as rank 2 tensor structure due to its pure cylindrical shape, which proves the good selectivity of the centre frequency in detecting thin cylindrical structure aimed for the micro-channels. 

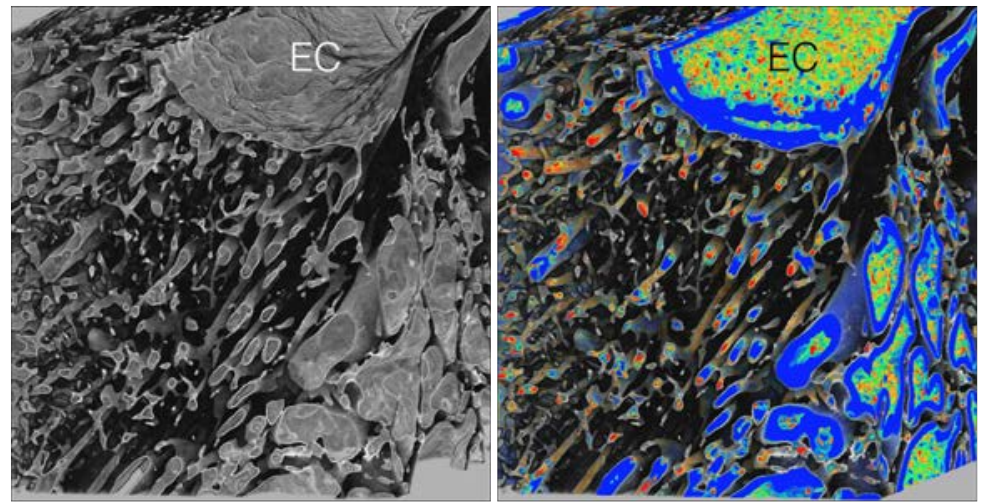

Figure 6.18: Left. Original dataset used for the study with a special transfer function to only visualize the transition between bone and other structures. Right. Results from the structure tensor analysis overlaid on the original data.

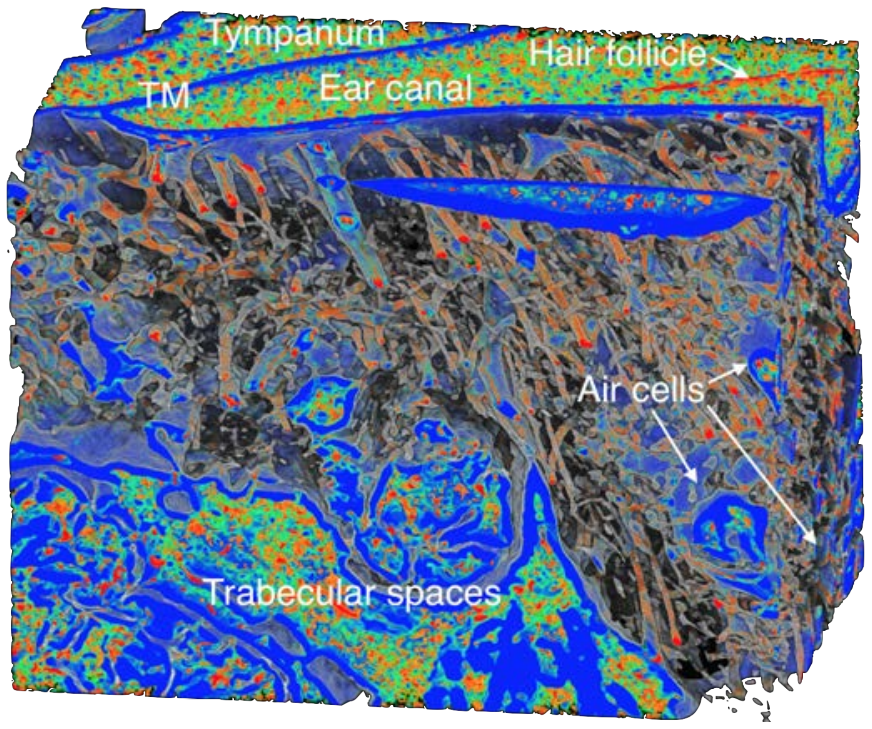

Figure 6.19: $3 \mathrm{D}$ representation of the resulting structure tensor analysis on the original data where only transition between bone and other structures is allowed. Legend. TM: tympanic membrane.

Figure 6.20 gives a non-exhaustive representation of micro-channels with various different shapes encountered during the analysis. Note that these subfigure A-H are of different magnification for the sole purpose of visualization.

Fig. 6.20(A) illustrates the perfect rank 2 tensor type structures within the 

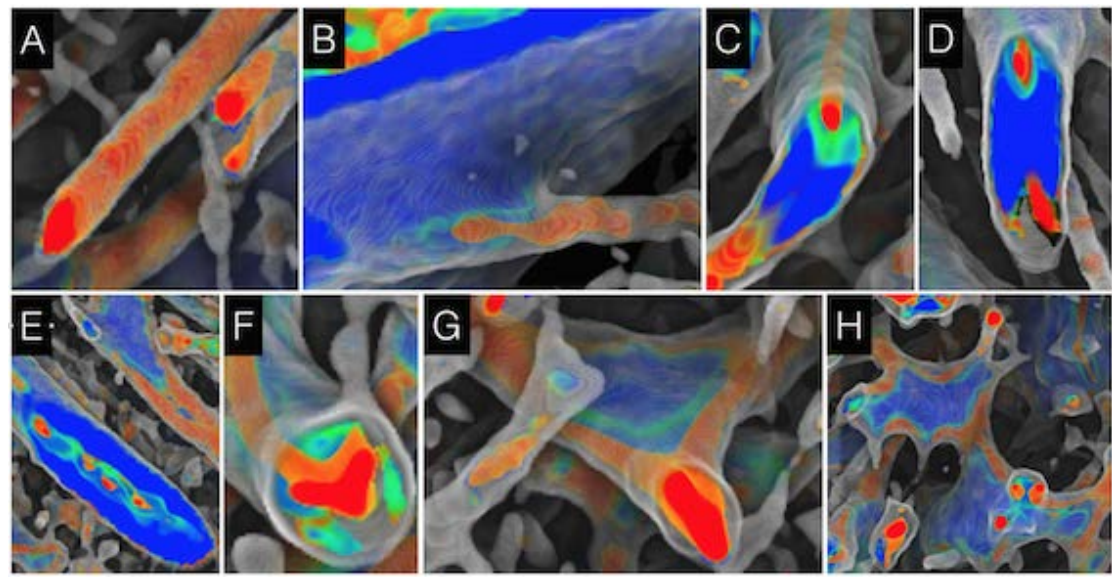

Figure 6.20: Micro-channels with different shapes.

micro-channels. Fig. 6.20(B) emphasizes the transition from a rank 1 tensor type structure to a rank 2 tensor type structure. Fig. 6.20(C) illustrates the intermix within a micro-channel of $p_{1}, p_{2}$ tensor type structures with occasionally a slight amount of $p_{3}$ tensor type structure.

Fig. 6.20(D) depicts a broader micro-channel with two rank 2 tensor type structures placed on either side of a micro-channel, with a well-defined rank 1 tensor type structure filling the gap in between. Fig. 6.20(E) reveals a flatten micro-channel with seemingly several rank 2 tensor type structures in the central part, and surrounded by a rank 1 tensor type structure. Fig. 6.20 (F) pictures the merging of two to three rank 2 tensor type structures into a larger rank 2 tensor type structure, at the center of a larger microchannel.

Fig. 6.20(G) presents a structure resembling a hub, never described before, receiving three micro-channels at its extremities with a rank 2 tensor type structure running along the edges and with a rank 1 tensor type structure at its centre. Fig. 6.20(H) describes a similar hub structure having more connections. As for the hub, presence of more than one rank 2 tensor type structures in a single micro-channel along with bifurcation or merging of rank 2 tensor type structures, have never been reported in the literature before.

Fig. 6.21 depicts several mastoid air cells partially cut (white stars). The second mastoid air cell, to the left, connects on its right side to a microchannel with a net transition from rank 1 tensor type to a rank 2 tensor type. On the opposite side of this micro-channel, the rank 1 tensor type structure lining the wall of the mastoid air cell (star to the right) penetrates the channel. 


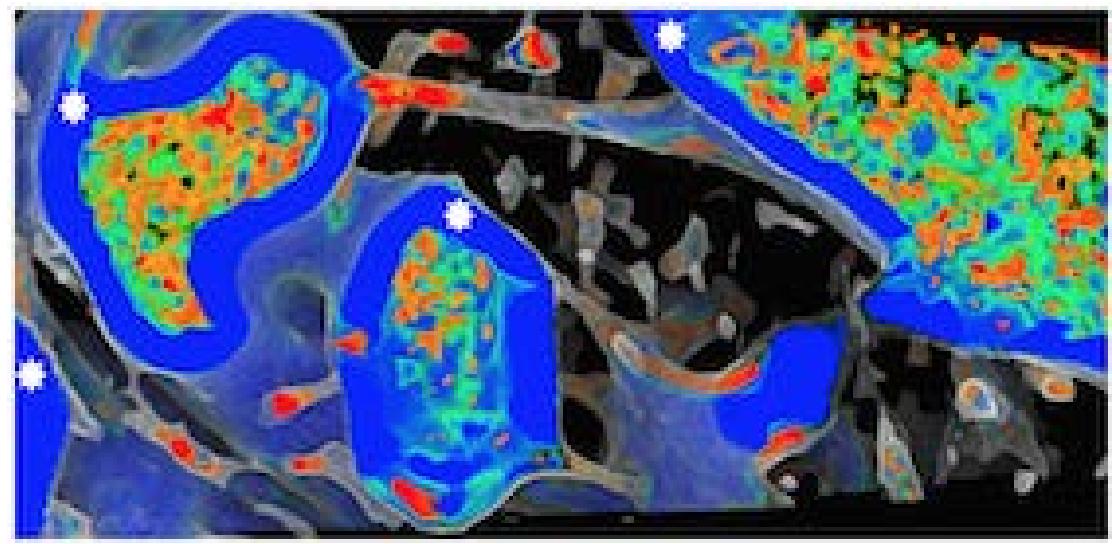

Figure 6.21: Micro-channels connecting to mastoid air cells (white stars).

The end-tip of the rank 1 tensor structure type indentation displays a transition toward a rank 2 tensor type structure, strongly indicating a red rank 2 tensor type structure in the missing part of the channel. The same applies to the lower right part of the middle mastoid air cell, where two micro-channels clearly connect to a hub structure.

This study has demonstrated the structural variation of contents inside the micro-channels by a local structure tensor analysis. From this analysis, discovery of unreported hub structures may help understand the origin and possible multi-role of this complex network formed by these micro-channels.

Presence of noise within the air cells, along with the missing information in some micro-channels, suggest the future need of image enhancement using an adaptive filtering technique based on the local structure tensor analysis used in this study. A larger scale study is also considered in the future in order to validate the method proposed in this pre-analysis.

This analysis is presented in Paper 3 and was presented as a poster at the International Symposium of Biomedical Imaging in New York in April 2015 . 


\section{Summary of Papers}

"Suum cuique Pulchrum."

To each its own is beautiful.

\subsection{Introduction}

This chapter provides brief summaries of the enclosed papers. Most of the material in the papers have been covered in the first part of the thesis.

\subsection{Paper I - Mastoid structural properties deter- mined by analysis of high-resolution CT scan- ning}

In this paper we describe the determination of the surface area to volume ratio, which may indicate that the mastoid air cell system is adapted to gas exchange, and assess the influence of the resolution on these parameters by downsampling the data four times. The surface area and volume were obtained through image processing tools commonly found in the field of hearing research, for comparison purposes though adjusted to the high resolution of the micro-CT scannings. The current study found significantly higher surface area and surface area to volume ratio than found in previous studies, performed at a limited resolution of around $625 \mu \mathrm{m}$ commonly found in clinical CT scanners. The statistics resulting from this study are important for a more accurate modeling of the middle ear physiology. 


\subsection{Paper II - Micro-channels in the mastoid anatomy. Indications of a separate blood supply of the air cell system mucosa by micro-CT scanning}

This paper describes a discovery within the temporal bone named after its structural shape, i.e. the micro-channels. A qualitative analysis using volume rendering first demonstrated the presence of these micro-channels connecting the surface of the compact bone directly to the mastoid air cells as well as forming a network of connections between the air cells. Then a quantitative analysis on $2 \mathrm{D}$ slices was employed to determine the average diameter of these micro-channels as well as their density at a localized area. These channels are believed to house a separate vascular supply for the mastoid mucosa. This discovery further help improving our knowledge of its physiological properties, which may have important implications for understanding of the pressure regulation of the middle ear.

\subsection{Paper III - Structural analysis of micro-channels in human temporal bone}

In this paper a preliminary structural analysis of the micro-channels was implemented using structure tensor analysis. The eigenvalues obtained from the estimated local structure tensor were then used to build probability maps representing planar, tubular, and isotropic tensor types. A visual interpretation of the three different tensor structure types was possible by assigning a respective RGB color, and the full structure tensor was rendered along with the original data to allow a smooth transition between these three tensor types. Such a structural analysis has provided new and relevant information about the micro-channels, but also their connections to the mastoid air cells. 
This chapter gives a discussion about the chronological history of this research. Some ideas for future work are also presented.

My first research was a clinical analysis about determining the surface area and volume of the mastoid air cell system from human temporal bone, using conventional clinical CT scanning and cone-beam CT. While the primary results were interesting, we realized that from dry bone specimens used for teaching, the number of mastoid air cells, and most importantly their size range, was beyond the possible resolution of a voxel resolution found in conventional clinical CT. Being aware of the cone-beam technology commonly used in an Ear Nose and Throat (ENT) department, data were collected on human temporal bone specimens. While cone-beam CT scanning do provide high resolution scan images, they contain a fairly large amount of noise that would hamper the small mastoid air cells.

Through contact with a centre of X-ray tomography located at the department of Physics and Astronomy at the university of Ghent (Belgium), we obtained amazing quality scans from eight temporal bone specimens, adjusted manually in size in order to optimize the scanning process. While assessing whether the temporal bone and most importantly whether the mastoid air cell system was fully visible in the field of view of the microCT scanning, we used powerful computers available in the department to perform some volume rendering of the scanned temporal bone. While adjusting the transfer function to restrict certain structures to be visible, we encountered some previously undescribed structures within the bone. Due to the availability of micro-CT scannings from other bones in the human body, such as a finger and a complete knee joint, we realized that these micro-channels were unique and possibly related to the temporal bone and more specifically to the mastoid air cell system.

Because the main idea using these micro-CT scannings were to investigate the surface area to volume ratio with a much broader range of mastoid air 
cell sizes, we decided to focus on this analysis. Such findings are more rare nowadays, we decided to look further into these micro-channels and describe some of the characteristics while trying to understand their potential role in relation to the mastoid air cell system and more importantly, their possible implications for further understanding of the pressure regulation of the middle ear.

Observing these micro-CT scans using volume rendering has enabled us to understand that these micro-channels do follow specific patterns. While some micro-channels run parallel in relation to each others, some have a more unstructured direction. Their multiple connections to singular mastoid air cells has further led to wonder about their exact purpose. This leads to the mixture where physiology starts to play a role along with the anatomy. Knowing that the mastoid air cells are lined with a mucosa, these micro-channels could possibly form a supplementary source for blood supply. Furthermore, where one end of these micro-channels is very often communicating with the mastoid air cell, the other end is often perceived as an indentation on the external surface of the mastoid bone, where soft tissues along with blood vessels are directly connected to the periosteum, the outer surface of the bone. Thus, our hypothesis suggest to provide blood to the mucosa of the mastoid air cells.

Spending a tremendous amount of hours visualizing these micro-CT scans, just for the sake of curiosity and for producing nice illustrations for oral presentations, I got more and more acquainted with the anatomy of the temporal bone, and started to get trained to suddenly see structures possibly never reported before. This is the case for the mucosal strands found in the mastoid air cells. Mucosal folds are known to surround the ossicles but to our knowledge so far, no one has reported them in the mastoid air cells. The tiny pores within the septae separating the mastoid air cells, despite the air cell conducts, is also opening our mind that the mastoid resemble more and more a fixed mini lung with common properties such as respiratory epithelium, gas exchange, and a large surface area in relation to a limited volume.

Overlaying the structure tensor analysis over the original data, while using volume rendering for visualization, allowed us to suddenly observe other structures like the triangular or star-shaped structure attached to three or more micro-channels that we know nothing of yet. These findings lead to further question whether these micro-channels are only tubular structures, or whether they are part of a bigger class of structures along with for instances sinusoids that could hypothetically be used as blood reserves needed for the mucosa lining the mastoid air cells, when the pressure in the ear is not equalized by thickening or shrinking.

Despite the huge challenge, this very complex blend of technical and med- 
ical knowledge lead to the discovery of new structures within the mastoid of human temporal bone. These findings add important knowledge to the immense puzzle describing the mastoid both in terms of anatomy but also from a physiological point of view, which makes this study even more exciting.

Among further ideas for future work, several paths are already planned. A first extension of this study is the writing of a clinical journal paper concerning the discovery of another structure within the mastoid bone, which could further relate the analogy of the mastoid air cell system with the human lungs. This process is already started. A possible collaboration with Dr. Zsuzsanna Csákányi working at the ENT department of Heim Pal Children's Hospital (Budapest, Hungary) could lead to the micro-CT scanning of one paediatric temporal bone with a history of chronic otitis media. This collaboration could bring important knowledge on the mastoid air cell system at early stage of age, but also how chronic otitis media affects the temporal bone from a structural point of view.

From a more technical aspect, a first path is to apply some adaptive filtering of the original data, to enhance the weak structures while keeping the other structures untouched before performing a structure tensor analysis on the data, which can be seen as an extension of Paper 3. As knowledge about the mastoid air cell system and newly discovered structures emerge, there is a increasing need to define the shape of the mastoid air cell system itself, as for instance what is an air cell and how their shape is influenced by surrounding structures and whether they can be clustered in regions as illustrated in existing literature such as in Mirko Tos book [44].

Another path is to estimate the surface area to volume ratio of the tympanum from the micro-CT scans, as was previously done for the mastoid air cell system in the first paper. This will allow us to assess how these two connected structures relate to each others. Along that line, another idea is to use a different approach to estimate the surface area to volume ratio of the overall airspaces from both the mastoid air cell system and the tympanum, while using a variant of the structure tensor analysis.

More ideas are present, but the above suggestions are believed to form a good basis for the remaining of the PhD program. 



\section{Bibliography}

[1] CT data configuration acquisitions. http://d32ogoqmya1dw8.cloudfront.net/ images/research_education/geochemsheets/techniques/_1172960774.png.

[2] Illustration of the eustachian tube at the level of nasopharynx. http://upload.wikimedia.org/wikipedia/commons/e/e3/Gray855.png.

[3] Lightspeed and brightspeed dicom conformance statement. https://www3.gehealthcare.se/media/documents/usglobal/products/interoperability/dicom/computed-tomography/gehcdicom-conformance_lightspeed-brightspeed_5198351-100_rev1.pdf.

[4] N. T. abd R.B. Pitts, I. Braun, and H. Heindel. Mastoid size determined with lateral radiographs and computerized tomography. Acta Otolaryngolica, 103:226-231, 1987.

[5] B. Ars and N. Ars-Piret. Middle ear pressure balance under normal conditions. specific role of the middle ear structure. Acta Otorhinolaryngol. Belg., 48:339-342, 1994.

[6] B. Ars, F. Wuyts, P. V. de Heyning, I. Miled, J. Bogers, and E. V. Marck. Histomorphometric study of the normal middle ear mucosa. Acta Otolaryngol. (Stockh), 117:704-707, 2006.

[7] E. Boulpaep. Arteries and veins. In: Boron, W.F., Boulpaep, E.L. (Eds.), Medical Physiology: A Cellular and Molecular Approach. Saunders Elsevier, Philadelphia, PA, 2009:467-469, 2009.

[8] D. M. L. Cooper, A. L. Turinsky, C. W. Sensen, and B. Hallgrimsson. Quantitative $3 \mathrm{~d}$ analysis of the canal network in cortical bone by micro-computertomography. The anatomical record (part B: New Anat.), 247B:169-179, 2003 .

[9] Z. Csákányi, G. Katona, E. Josavai, F. Mohos, and I. Sziklai. Volume and surface of the mastoid cell system in otitis media with effusion in children: a case-control study by three-dimensional reconstruction of computed tomographic images. Otology \& Neurotology, 32:64-70, 2011.

[10] J. Dirckx, Y. Marcusohn, M. Gaihede, S. I. Puria, R. Fay, and A. Popper. Quasi-static pressures in the middle ear cleft. The Middle Ear: Science, Otosurgery, and Technology, 93 Springer Handbook of Auditory Research 46, pages $93-133,2013$.

[11] W. Doyle. Increases in middle ear pressure resulting from counter-diffusion of oxygen and carbon dioxide into the middle ear of monkeys. Acta Otolaryngol., $117(5)$.

[12] W. Doyle. Mucosal surface area determines the middle ear pressure response following establishment of sniff-induced underpressures. Acta Otolaryngol., 119(6). 
[13] R. Drebin, L. Carpenter, and P. Hanrahan. Volume rendering. Computer Graphics, 22(4).

[14] A. Eklund, M. Andersson, and H. Knutsson. True 4D image denoising on the GPU. International Journal of Biomedical Imaging, 2011.

[15] R. C. Gonzalez, R. E. Woods, and S. L. Eddins. Digital Image Processing using MATLAB. Pearson Prentice Hall, 2004. ISBN 0130085197.

[16] G. Granlund and H. Knutsson. Signal Processing for Computer Vision. Kluwer Academic Publishers, 1995. ISBN 0792395301.

[17] E. Hentzer. Histological studies of the normal mucosa in the middle ear, mastoid cavities and eustachian tube. Annals of Otology, Rhinology and Laryngology, 79:825-833, 1970.

[18] J. Holmquist. Aeration in chronic otitis media. Clinical Otolaryngology, 3(3):279-284, 1978.

[19] N. Ikonomatakis, K. Plataniotis, M. Zervakis, and A. Venetsanopoulos. Region growing and region merging image segmentation. In Digital Signal Processing Proceedings, 1997. DSP 97., 1997 13th International Conference on, volume 1, pages 299-302, 1997.

[20] M. Isono, K. Murata, H. Azuma, M. Ishikawa, and A. Ito. Computerized assessment of the mastoid air cell system. Auris Nasus Larynx, 26(2):139145, 1999.

[21] B. Jähne. Digital Image Processing. 5th revised and extended edition. 2002. ISBN 3540677542.

[22] M. K. Kalra, M. M. Maher, T. L. Toth, B. Schmidt, B. L. Westerman, H. T. Morgan, and S. Saini. Techniques and applications of automatic tube current modulation for CT. Radiology, 233(3):649-657, 2004.

[23] H. Knutsson. Filtering and Reconstruction in Image Processing. PhD thesis, Linköping University, 1982.

[24] H. Knutsson, M. Andersson, and J. Wiklund. Advanced filter design. In Scandinavian conference on image analysis (SCIA), 1999.

[25] H. Knutsson and M. T. Andersson. Optimization of sequential filters. In In Proceedings of the SSAB Symposium on Image Analysis, pages 87-90, 1995.

[26] G. Läthén. Level Set Segmentation and Volume Visualization of Vascular Trees. PhD thesis, 2013. ISBN 03457524.

[27] D. Lee, B. Jun, D. Kim, M. Jung, and S. Yeo. Volume variation of mastoid pneumatization in different age groups: a study by three-dimensional reconstruction based on computed tomography images. Surg Radiol Anat, $27: 37-42,2005$.

[28] W. E. Lorensen and H. E. Cline. Marching cubes: A high resolution 3D surface construction algorithm. ACM Computer Graphics, 21:163-169, 1987.

[29] M. Luntz, S. Malatskey, M. Tan, E. Bar-Meir, and D. R. D. Volume of mastoid pneumatization: three-dimensional reconstruction with ultrahighresolution computed tomography. Ann Otol Rhinol Laryngol, 110 (5Pt1):486490, 2001.

[30] S. Mansour, J. Magnan, H. Haidar, K. Nicolas, and S. Louryan. Comprehensive and Clinical Anatomy of the Middle Ear. 2013. ISBN 3642369677. 
[31] Y. Marcusohn, A. Ar, and J. Dirckx. Perfusion and diffusion limitations in middle ear gas exchange: The exchange of $\mathrm{CO} 2$ as a test case. Hear. Res., 265:11-14, 2010.

[32] B. Masschaele, V. Cnudde, M. Dierick, P. Jacobs, L. V. Hoorebeke, and J. Vlassenbroeck. UGCT: New x-ray radiography and tomography facility. Nucl. Instrum. Meth., A 580:266-269, 2007.

[33] B. Masschaele, M. Dierick, D. V. Loo, V. Cnudde, M. N. Boone, L. Brabant, E. Pauwels, V. Cnudde, and L. V. Hoorebeke. HECTOR: A 240kv micro-CT setup optimized for research. Journal of Physics: Conference Series, 463 (1):1-4, 2007.

[34] R. Matanda, P. V. D. Heyning, J. Bogers, and B. Ars. Behaviour of middle ear cleft mucosa during inflammation: Histo-morphometric study. Acta OtoLaryngologica, 126:905-909, 2006.

[35] W. W. Mayo-Smith, A. K. Hara, M. Mahesh, D. V. Sahani, and W. Pavlicek. How i do it: Managing radiation dose in CT. Radiology, 273(3):657-672, 2014.

[36] M. Miura, H. Takahashi, I. Honjo, S. Hasebe, and M. Tanabe. Influence of the gas exchange function through the middle ear mucosa on the development of sniff-induced middle ear diseases. The Laryngoscope, 108:683-686, 1998.

[37] M. Park, S. Yoo, and D. H. Hoon. Measurement of surface area in the human mastoid air cell system. J. Laryngol. Otol., 114:93-96, 2000.

[38] S. P. Raman, M. Mahesh, R. V. Blasko, and E. K. Fishman. CT scan parameters and radiation dose: practical advice for radiologists. Journal of the American College of Radiology : JACR, 10(11):840-846, 2013.

[39] J. Sadé and A. Ar. Middle ear and auditory tube: Middle ear clearance, gas exchange, and pressure regulation. Otolaryngol. Head Neck Surg., 116:499$524,1997$.

[40] M. Sezgin and B. Sankur. Survey over image thresholding techniques and quantitative performance evaluation. Journal of Electronic Imaging, 13(1):146-165, 2007.

[41] J. Swarts, B. C. Doyle, C. A. CM, and W. Doyle. Surface area-volume relationships for the mastoid air cell system and tympanum in adult humans: Implications for mastoid function. Acta oto-laryngologica., 130(11):12301236, 2010.

[42] J. Swarts, S. Folay, C. Alper, W. Doyle, and J. William. Mastoid geometry in a cross-section of humans from infancy through early adulthood with a confirmed history of otitis media. International journal of pediatric otorhinolaryngology, 76(1):137-141, 2012.

[43] A. Telser, J. Young, and K. Baldwin. Elsevier's Integrated Histology. Mosby/Elsevier. Inc. Philadelphia, 2007. ISBN 0323033881.

[44] M. Tos. Manual of Middle Ear Surgery. Vol.2: Mastoid Surgery and Reconstructive Procedures. Georg Thieme Verlag, 1995. ISBN 0865775893.

[45] J. Vlassenbroeck, M. D. B. Masschaele, V. Cnudde, L. V. Hoorebeke, and P. Jacobs. Software tools for quantification of x-ray microtomography at the UGCT. Nuclear Instruments and Methods in Physics Research, A(580):442$445,2007$. 
[46] J. Vrabec, S. Champion, J. G. R. Johnson, and G. Chaljub. 3D CT 534 imaging method for measuring temporal bone aeration. Acta Otolaryngol, 122:831-835, 2002.

[47] Wikipedia. Photo of inside a CT scan gantry. https://upload.wikimedia.org/wikipedia/commons/7/76/Ct-internals.jpg.

[48] J. Yi and J. B. Ra. A locally adaptive region growing algorithm for vascular segmentation. Int. J. Imaging Systems and Technology, 13:208-214, 2003.

[49] J. Zhang, C.-H. Yan, C.-K. Chui, and S.-H. Ong. Fast segmentation of bone in CT images using 3d adaptive thresholding. Computers in Biology and Medicine, 40(2):231-236, 2010.

[50] C. Zollikofer and J. Weissmann. A morphogenetic model of cranial pneumatization based on the invasive tissue hypothesis. The Anatomical Record, WILEY-LISS, INC., 291:1446-1454, 2008. 


\section{Papers}

The articles associated with this thesis have been removed for copyright reasons. For more details about these see:

http://urn.kb.se/resolve?urn=urn:nbn:se:liu:diva-122179 